\title{
Advanced Methods of Electron Microscopy in Catalysis Research
}

\section{Miguel José-Yacamán*, Arturo Ponce*, Sergio Mejía-Rosales ${ }^{\dagger}$, Francis Leonard Deepak}

*Department of Physics and Astronomy, University of Texas at San Antonio, One UTSA Circle, San Antonio, Texas 78249, USA.

${ }^{\dagger}$ Center for Innovation and Research in Engineering and Technology, and CICFIM-Facultad de Ciencias Físico-Matemáticas, Universidad Autónoma de Nuevo León, San Nicolás de los Garza, NL 66450, México

${ }^{\ddagger}$ The International Iberian Nanotechnology Laboratory, Av. Mestre José Veiga-4715-310, Braga, Portugal

\section{Contents}

1. Introduction 280

2. STEM and TEM 280

2.1. The Principles of TEM and STEM Imaging 282

2.2. HAADF-STEM 285

$\begin{array}{ll}\text { 2.3. Aberrations } & 287\end{array}$

2.4. Practical Guide for STEM Imaging 291

3. Examples of Applications to Catalyst Characterization 294

3.1. Metal Catalysts on a Crystalline Substrate 294

3.2. Metals on a Noncrystalline Substrate 299

$\begin{array}{ll}\text { 3.3. Metals on } \gamma-\mathrm{Al}_{2} \mathrm{O}_{2} & 301\end{array}$

3.4. Bimetallic Nanoparticles and Clusters 304

3.5. Understanding the Structure of Bimetallic Nanoparticles and Clusters Using 305 Advanced Electron Microscopic Techniques

3.6. In Situ Heating Experiments of Bimetallic Nanoparticles and Clusters 316

3.7. Multimetallic Systems 318

$\begin{array}{ll}\text { 3.8. Conclusions } & 318\end{array}$

4. Electron Microscopy of Layered Materials 319

$\begin{array}{ll}\text { 4.1. Introduction } & 319\end{array}$

4.2. $\mathrm{MoS}_{2}$ Nanowire Catalysts $\quad 320$

4.3. Co-Doped $\mathrm{MoS}_{2} / \mathrm{WS}_{2}$ Nanowire Catalysts 326

4.4. $\mathrm{MoS}_{2}$ Nanoplates $\quad 334$

$\begin{array}{ll}\text { 4.5. Conclusions } & 337\end{array}$

Acknowledgments $\quad 338$

References $\quad 338$ 


\section{INTRODUCTION}

The study of catalytic systems has been one of the most active areas of application of electron microscopy. Indeed, a typical catalyst is made of small particles - in the size interval of nanometers to even single atoms - and a substrate that is usually polycrystalline or, in a few cases, amorphous. The catalytic particles are made either of noble or transition metals, or oxides that strongly scatter electrons and therefore produce a high contrast in the micrograph.

Most of the commercially interesting supports are also oxides or semigraphitic carbon, which also produce high contrast; therefore, the problem of the observation of a catalytic nanoparticle through an electron microscope is reduced to separate its contrast from that of the substrate. In a few important cases, the catalysts are not even nanoparticles but are nanoribbons, such as those made of $\mathrm{MoS}_{2}$, that in principle are expected to behave as semiconductors.

\section{STEM AND TEM}

Conventional transmission electron microscopy (TEM) is a beam characterization technique with almost 80 years since the development of the first microscope. In TEM, a large area of the specimen is illuminated, the magnification is performed by the lens system underneath the specimen, and subsequently the whole image is registered instantaneously. Conventional scanning electron microscopy (SEM) was designed after the TEM and has been widely used for surface analyses in biological and materials sciences. Scanning transmission electron microscopy (STEM) combines both operational modes. In STEM mode, a convergent electron beam is focused to a small area of the sample. To register an image, the electron probe is raster-scanned and subsequently propagated through the sample. Due to the electron-matter interaction, the trajectory of the electrons is scattered and different kinds of signals are registered in sync with the electron probe scanning. STEM resolution is determined primarily by the size of the probe. One of the main advantages of STEM over TEM is that the images generated by the electrons scattered out to high angles on a high-angle annular dark-field (HAADF) detector are chemically sensitive, and a sample with a definite crystalline arrangement is not necessarily a requirement. The capacity of STEM to generate these 
different levels of contrast is commonly known as $Z$-contrast; the contrast dependence goes approximately as $Z^{\sim 2}$, with $Z$ corresponding to the atomic weight of the element that caused the scattering of the electrons (Pennycook, 1989).

TEM and STEM operational modes are necessary for the study of nanoparticles. The appropriate generation and interpretation of electron micrographs is crucial for this purpose. The interpretation of the electron micrographs is not always straightforward because the intensity signal that correlates with the atomic positions depends not just on the length of the atomic columns parallel to the direction of the electron beam, but also on the chemical species and the microscope's parameters at which the micrograph was obtained. In this way, the problem of extracting a third dimension from the information contained in a strictly two-dimensional (2D) image requires the comparison of the observed images against simple models acquired by previous experience or inherited, and in many cases a theoretical model of the structure is necessary.

In TEM, the information transfer in phase-contrast imaging is determined by the objective lens and the size of its aperture, which are directly related to the temporal and spatial coherence of the electrons. In Figure 4.1, a conventional high-resolution transmission electron microscopy (HR-TEM) micrograph of a bimetallic nanoparticle $2 \mathrm{~nm}$ in diameter shows the interatomic distances; however, the contrast is not directly related with the different atomic positions in the bimetallic nanoparticle (Corthey et al., 2010) proposed by the theoretical model shown in Figure 4.1b.
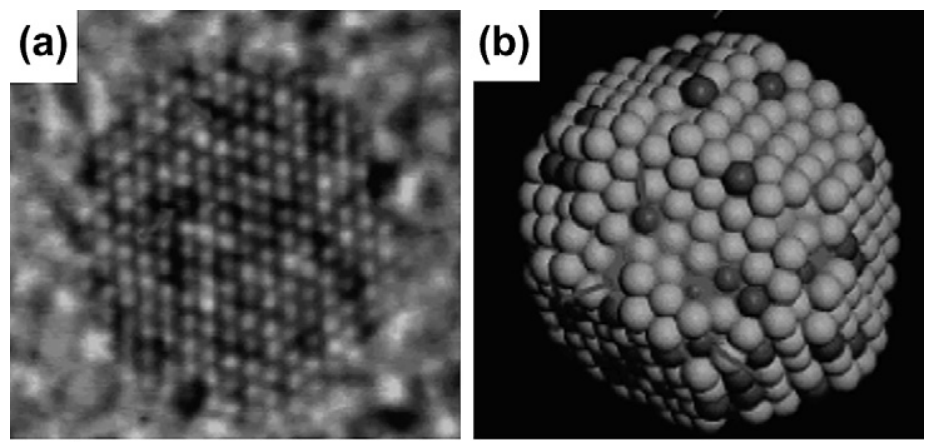

$5 \mathrm{~nm}$

Figure 4.1 (a) HR-TEM image of a bimetallic gold-palladium nanoparticle. (b) Theoretical model of the bimetallic nanoparticle. 

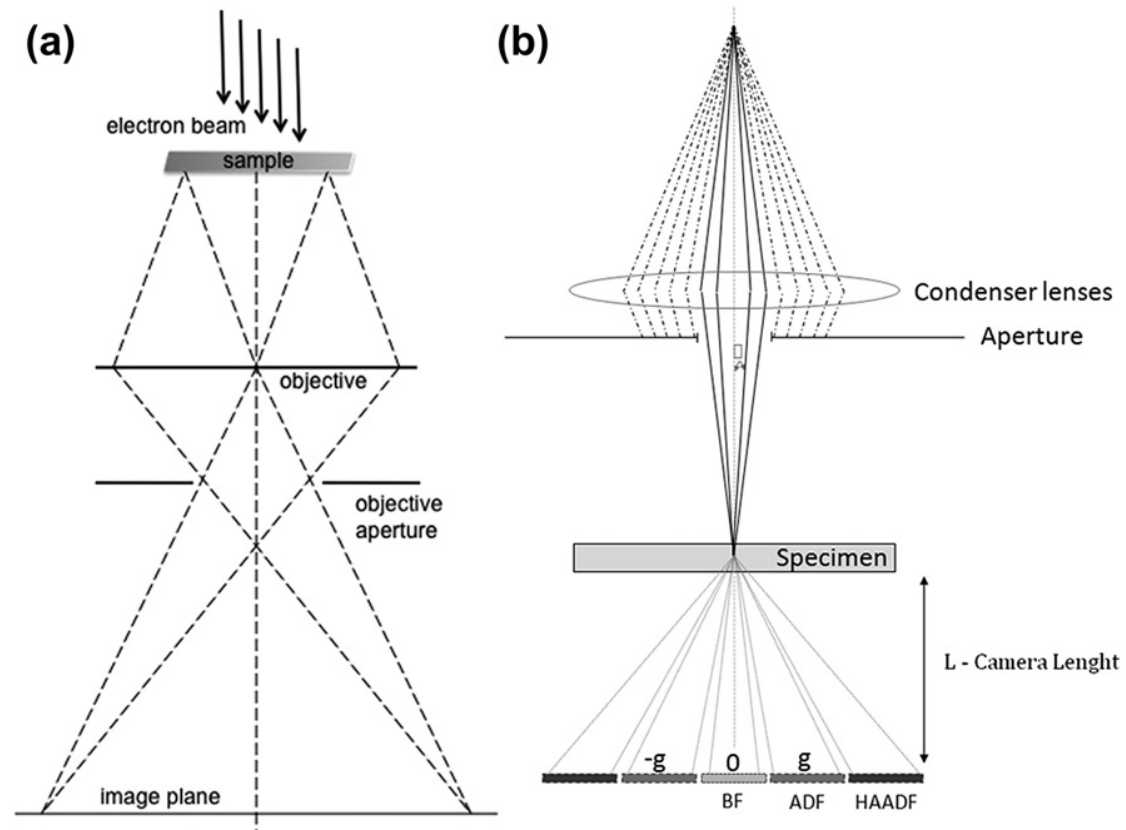

Figure 4.2 Simplified diagram of the (a) TEM and (b) STEM operational modes.

The optical arrangement of a conventional TEM compared with the STEM configuration is illustrated in the schematic diagram shown in Figure 4.2.

\subsection{The Principles of TEM and STEM Imaging}

Unlike macroscopic objects, whose structural properties can be inferred directly by investigating how a beam of light interacts with the object, the study of matter at the nanoscale requires investigating how a beam of electrons interacts with the sample, since wavelengths on the order of Ångstroms (or even smaller) are required to reach this level of resolution. This electron beam is produced by accelerating the electrons by applying a potential difference of several kilovolts to an emitter, usually a field emission cold cathode; other electron sources use thermionic emission to overcome the work function of the cathode. Originally, the beam is not monochromatic but an energy-selecting spectrometer can be added to the electron gun, at the price of diminishing the beam current. A first electrostatic lens called Wehnelt makes the beam reaching the anode more convergent (Rose, 1990). An aperture on the anode plate allows 
the beam to actually get into the microscope. A field emission gun (FEG) actually has two anodes: the first one in charge of producing the beam, the second one in charge of accelerating the electrons (Reimer and Kohl, 2008).

When the beam produced by the electron gun interacts with a sample, different signals are generated. Most of the electrons are transmitted through the sample with just a small deviation from the optic axis (the original trajectory of the electron beam); when collected, this signal is used to produce bright-field (BF) images. Signals transmitted at higher angles can be collected using an angular detector to produce dark-field (annular dark-field [ADF], HAADF) images or the energy of the electrons can be measured to generate electron energy-loss spectroscopy (EELS) maps. The chemical composition of the sample can be investigated by analyzing the X-rays generated when the electrons in the sample are excited by the beam-what is called energy-dispersive X-ray (EDX) spectroscopy. Auger electrons can be generated by a somewhat complex process: The beam causes the ejection of an inner-shell electron of the specimen; the formed vacancy is filled by an electron of a higher level and the excess energy liberates a third electron, called an Auger electron.

TEM is a powerful technique to characterize the structure and chemical composition of condensed matter. Conventional TEM (CTEM) includes basically three operation modes: diffraction contrast (BF and dark-field $[D F])$, phase-contrast (HR-TEM), and electron diffraction (ED). These three operating modes provide information about the crystalline structure in the materials, morphology, structural defects, and even chemical composition. Complementing CTEM, it is also possible to use STEM. Figure 4.2 shows the trajectory of the electrons in a TEM for both the TEM and STEM modes. Both modes are based on the interaction of an electron beam, generated by an electron gun at the top of the microscope, with the lenses and the specimen.

BF-CTEM is the simplest and most frequently used mode of operation of a TEM. In this mode, the images are formed by the direct (nondiffracted) electron beam, while an aperture blocks the diffracted beam. In these images, regions with no particles (and maybe just the carbon grid) appear as bright. DF images are generated by a detector that collects only the diffracted electrons. The regions with no particles appear dark-hence the name dark field. While in DF micrographs contrast is enhanced, BF images require a less-intense beam; thus this technique is less intrusive. 
In high-resolution electron microscopy, the image can be considered the result of the interference of the transmitted and the diffracted beams after the interaction with the sample. Thus, the sample must be thin enough to transmit the electrons. When nanoparticles are being prepared for analysis by TEM or STEM, it is common to prepare the specimen by placing the nanoparticles on holey carbon copper grids. In some synthesis procedures, the nanoparticles are covered with a layer of organic ligands (Corthey et al., 2010); if required, this organic layer can be removed by exposing the specimen to a beam shower for a few minutes before placing it in the microscope.

At the range of operating voltages of modern electron microscopes, the electron's momentum is very large-large enough that it is necessary to take into account relativistic corrections to describe correctly the interactions (Carter and Williams, 2009). The equation that considers the interaction of the electrons with the specimen and lenses requires some adjustments on the values of mass and wavelength. In a strict formalism, the equation that governs these interactions is the relativistic Dirac equation, but it is customary to use instead the approximated, easier-tomanipulate Schrödinger equation since the Dirac equation is very difficult to deal with, even in the simplest cases. In its most basic version, the role of the specimen is represented in the Schrödinger equation by its projected electrostatic potential. For sake of simplicity in this brief description, we can assume a periodicity condition in this potential (as if being produced by a periodic crystal). Let this potential be represented by $\Phi(r)$. The specific details of $\Phi(r)$ depend mainly on the chemical composition and structural features of the specimen, but a better description would also include thermal effects. The Schrödinger equation that describes the interaction of this potential with an electron of mass $m$ and charge $e$ coming from the source is

$$
\left[-\frac{h^{2}}{8 \pi^{2} m} \nabla^{2}-e \phi(r)\right] \Psi(r)=E \Psi(r),
$$

and the wave function of the electron wave before interacting with the specimen can be described by using a simple plane wave:

$$
\Psi_{0}(r)=\exp \left\{i\left(\omega t-2 \pi k_{0} \cdot r\right)\right\} .
$$

The solution to Eq. (1) describes the electron wave function after the interaction of the beam with the specimen. In practice, the task of finding a solution requires a set of approximations in addition to the periodicity 
assumption and the plane wave nature of the incoming beam, such as assuming that the specimen is not very thick.

The objective lens is positioned just after the sample in a TEM and it focuses the electron beam; the role of the objective lens can be described mathematically by the Fourier transform of the electron beam wave at the exit of the sample. Unlike the lenses used in visible light microscopes, both the objective and corrector lenses in the electron microscope are made of a magnetic field generated by an electric current circulating a coil, which affects the trajectory of the electron beam. In an ideal microscope with no aberrations, the image observed will be directly related to the squared modulus of the image-plane wave function (Reimer and Kohl, 2008). But in the real world, the objective lens is not perfect and introduces aberrations in the image-plane wave function. The most recent models of high-resolution microscopes include in their design additional coils that act as aberration correctors that make sub-Ångstrom resolution possible.

\subsection{HAADF-STEM}

From the standpoint of the electron beam, a STEM is an inverted TEM in the sense that the trajectory followed by the beam through the optical components of the STEM is the same as in the TEM but in the opposite direction. The reciprocity theorem states that the propagation of the beam is time reversible, and thus if in the TEM the detector is exchanged with the FEG, the system becomes basically a BF-STEM. A consequence of this equivalence is that, unlike with CTEM, the electron beam interacts with only a small section of the sample at a time (a pixel in the image), and so a scan process, made by deflecting the beam using a set of coils, must be used to generate the image of the entire specimen. The width of the beam (the probe) greatly determines the resolution of the STEM, and $C_{s}$ correction is mandatory to obtain sub-Ångstrom resolution.

The scattered electrons in STEM mode can be registered by three different detectors. The BF detector collects the electrons transmitted in the path of the beam close to the optical axis, the BF electrons containing the total beam current. The annular dark-field (ADF) and the HAADF detectors are used to record the electrons scattered out of the path of the beam. In the STEM, the objective lens - and all the relevant optics - are positioned before the specimen. The scattered angle of the ADF detector is set around $\sim 40 \mathrm{mrad}$ and the outer angle is set around 60-200 mrad 
(see Figure 4.2). STEM differs from CTEM in that the electron beam interacts with only a small section of the sample; the scanning process is the one in charge of generating the image as a whole, whereas in TEM, a broad beam is interacting instantaneously with the sample. X-ray energy-dispersive spectroscopy (EDS) attached to the STEM mode provides an elemental analysis directly from the point or line rasterscanned in the sample. Therefore, the imaging using the BF or HAADF detectors in a complementary way can be matched to the EDS information, and the elemental mapping at atomic resolution is obtained. Figure 4.3 shows the setup of the STEM mode including the detectors previously mentioned. HAADF and BF images are indicated on the right side of Figure 4.3, and both can be acquired simultaneously. L corresponds to the camera length, which is the effective distance or magnification between the specimen and the detector plane position. The X-ray detector is included in Figure 4.3; the spectra can be collected at the same they are line scanned to register the STEM images.
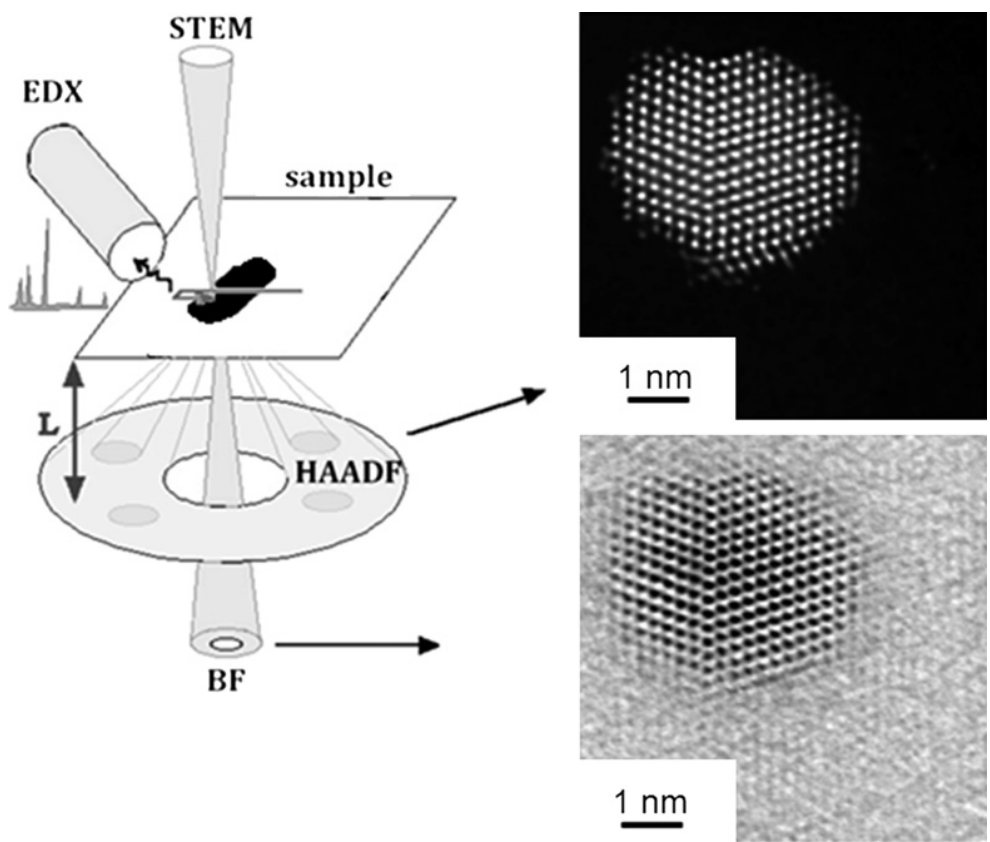

Figure 4.3 STEM setup, including the EDS spectrum detector. $L$ corresponds to the camera length. 


\subsection{Aberrations}

Aberrations are artifacts produced by the imperfections on the equipment. We have already described correction of chromatic aberration before the interaction of the electron beam with the specimen. Astigmatism and coma can be easily corrected using magnetic quadrupoles (Koch, 2002). Spherical aberrations, denoted $C_{s}$, are far harder to correct, but the corrections are necessary to produce images of atomic resolution. The objective lens, located behind the specimen in a TEM, focuses the electron beam, which mathematically can be described by the Fourier transform of the wave at the exit of the sample. Imperfections in the objective lens are the source of $C_{s}$, and additional optics is needed after this element to make the appropriate corrections.

The effect of $C_{s}$ can be appropriately explained in terms of the contrast transfer function (CTF). In short, the CTF is used to determine how the different spatial frequencies will be represented in a TEM image. The electron wave function at the image plane will be related to the CTF applied to the wave function only at the exit of the sample. In a spatial frequency range where the CTF is approximately constant, the distances and sizes of objects will be represented directly on the micrograph, while in a range of spatial frequencies where the CTF varies greatly, it would be quite difficult to determine whether a bright spot in the micrograph corresponds to the presence or absence of an atom. Objective apertures, the spatial coherence of the electron beam, and its wavelength will affect the CTF. Its definition is

$$
C T F(k)=-\sin \left[\frac{\pi}{2} C_{s} \lambda^{3} k^{4}+\pi \Delta z \lambda k^{2}\right] .
$$

Here, $C_{s}$ is the spherical aberration of the objective lens and $\Delta z$ is its defocus. A setting of particular interest is

$$
\Delta z=-\sqrt{4 / 3} C_{s} \lambda .
$$

called the Scherzer condition. At the Scherzer defocus, the CTF does not change its sign in a large spatial frequency range; hence all distances in this range can be interpreted directly as they appear in the image (Reimer and Kohl, 2008; Carter and Williams, 2009).

Figure 4.4 shows the results of conventional HR-TEM simulations of a section of a gold lattice at different values of defocus, Scherzer included $(-50 \AA)$. The voltage was $200 \mathrm{kV}$ in all cases, and a small aberration of 

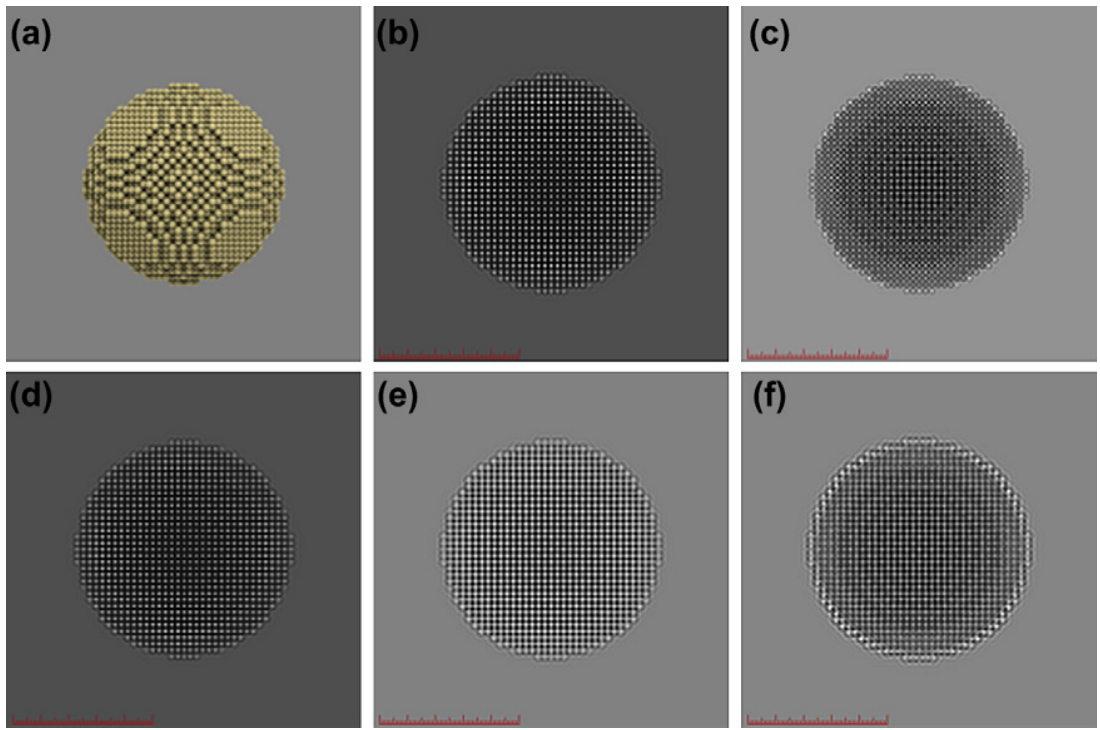

Figure 4.4 HR-TEM simulations of a spherical fcc gold nanostructure, calculated at different defocus values. (a) Model of the particle; (b) $\Delta z=-100 \AA$; (c) $\Delta z=-50 \AA$; (d) $\Delta z=0 \AA$; (e) $\Delta z=50 \AA$; (f) $\Delta z=100 \AA$; The image labeled as (c) corresponds to the Scherzer defocus. (See the color plate).

$0.01 \mathrm{~mm}$ was used with zero astigmatism. At Scherzer defocus, the atomic columns are completely resolved, even at the surface of the structure.

Aberration correction in TEM highly enhances the spatial resolution to such a degree that it is even possible to reach sub-Ångstrom level. The implementation of multipole corrector lenses in the electron optics system was developed in the 1990s, when the first correctors of the spherical and chromatic aberrations appeared. Probably the best way to acquire a sense of the neefor $C_{s}$ correction to reach sub-Ångstrom resolution is by comparing micrographs obtained under the same conditions except for the spherical aberration. This is, of course, impossible to do in practice, but it is possible instead to compare the results of TEM simulations at different values of $C_{s}$. Figure 4.5 shows a comparison that illustrates this.

The Z-contrast technique works remarkably well in metal nanoparticles because the intensity dependence on atomic number is close to $Z^{3 / 2}$, with minimum dependence on microscope defocus (Wall, 1974). This is definitely different from what is expected of BF imaging, where the signal varies 


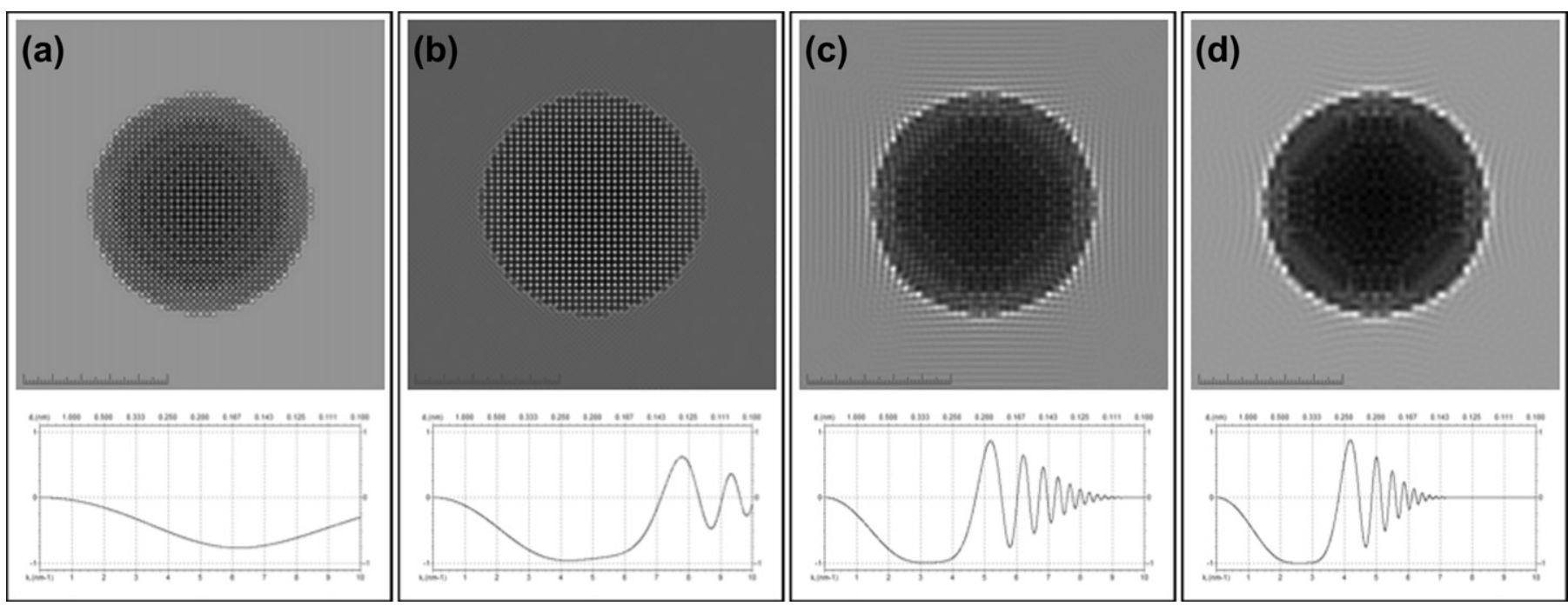

Figure 4.5 Simulated TEM micrographs calculated at several values of $C_{s}$ and their corresponding contrast transfers functions. (a) $0.01 \mathrm{~mm}$; (b) $0.1 \mathrm{~mm}$; (c) $0.5 \mathrm{~mm}$; (d) $1.2 \mathrm{~mm}$. 

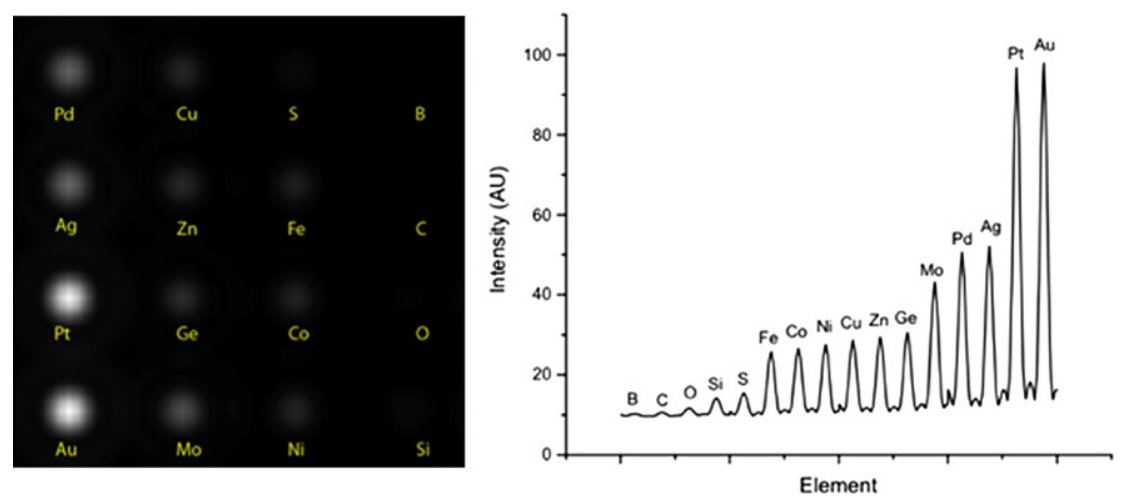

Figure 4.6 Profiles of intensity obtained from a series of HR-TEM simulated images (at a defocus of $-41 \mathrm{~nm}$ ). Note the intensity variation with the different elements. In each case, the columns of elements have the same number of atoms. (For color version of this figure, the reader is referred to the online version of this book).

weakly and nonmonotonically with Z. In HAADF-STEM, the differences among metals are evident. Figure 4.6 shows a Z-contrast STEM simulation of single atoms of different elements arranged in a $4 \times 4$ matrix. The line scan through the center of the atomic positions is shown at the right of the simulated image. The trend follows approximately a $Z^{1.46}$ relation, very close to the dependence expected by Pennycook and Boatner (Pennycook, 1998).

When used to image metal nanoparticles, $200 \mathrm{kV}$ is a common operation voltage in an HAADF-STEM, although lower voltages would allow investigation of the presence of molecules on the nanoparticle surface and a deeper understanding of the structure of the support matrix surrounding the nanoparticle. The HAADF detector is set normally at an inner angle 50 mrad, and the outer angle is set $\sim 100-200 \mathrm{mrad}$.

STEM-HAADF is extremely useful for size determinations in catalysts. This is exemplified in Figure 4.7. Several STEM micrographs of gold nanoparticles were used to generate a histogram of sizes. Figure 4.7a shows one of these micrographs. The particles, well distributed on the substrate, are easily recognized by the software once a threshold value of intensity and a minimum particle size are defined; Figure $4.7 \mathrm{~b}$ shows the histogram. In this example, the statistics gave a mean size of $2.3 \mathrm{~nm}$, with a standard deviation of $0.49 \mathrm{~nm}$. Several factors may affect the confidence on the size measurements, such as out-of-focus regions in the image or a low contrast level. This kind of procedure is extremely useful in catalytic research. 

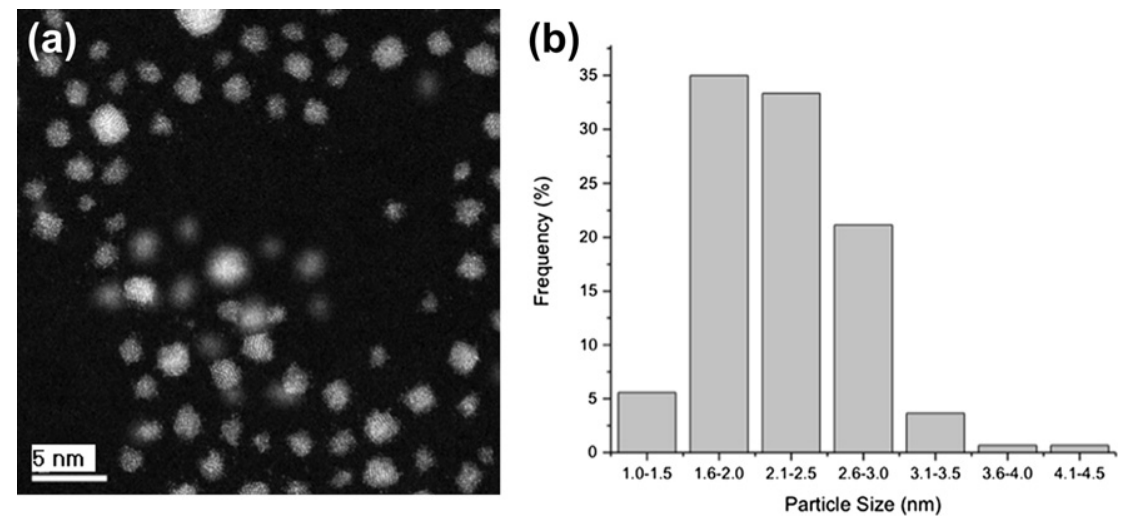

Figure 4.7 Histogram of sizes of gold particles obtained using STEM-HAADF images. Since no delocalization is produced on the image, the size distribution is more accurate.

\subsection{Practical Guide for STEM Imaging}

The basic parameters described in this section are used to register highresolution STEM images. The process must be started in TEM mode for the selection of the field of view and to adjust the crystal orientation, if needed. The eucentric focus must be adjusted with the $z$-control. By following the TEM initial setup, the system can be switched to STEM. The illumination focused on the sample must be adjusted by varying the size of the condensed aperture, the spot size, and the camera length $(L)$. Camera length is the effective distance or magnification between the specimen and the detector plane position. The collection angle depends on microscope camera length. Typical collection angle values for ADF and HAADF detectors are close to 40 and $200 \mathrm{mrad}$, respectively.

The resolution in STEM depends on the spot size of the electron beam. Spot size and the aperture of the condenser lens influence the beam current density in the sample and, as a consequence, on the resolution of the instrument. The beam current density in the sample can be increased either by a large aperture of the condenser lens or by using a lower spot size. Figure 4.8 shows two sketches for the rays under two different settings for STEM. Increasing the spot size (spot number) leads to increased demagnification of the source and a decreased current in the beam. Increasing the spot number leads to a reduction of the beam current.

Changing the size of the condenser lenses CL1 and CL2 also changes the beam current. Figure 4.9 sketches the rays corresponding to two 


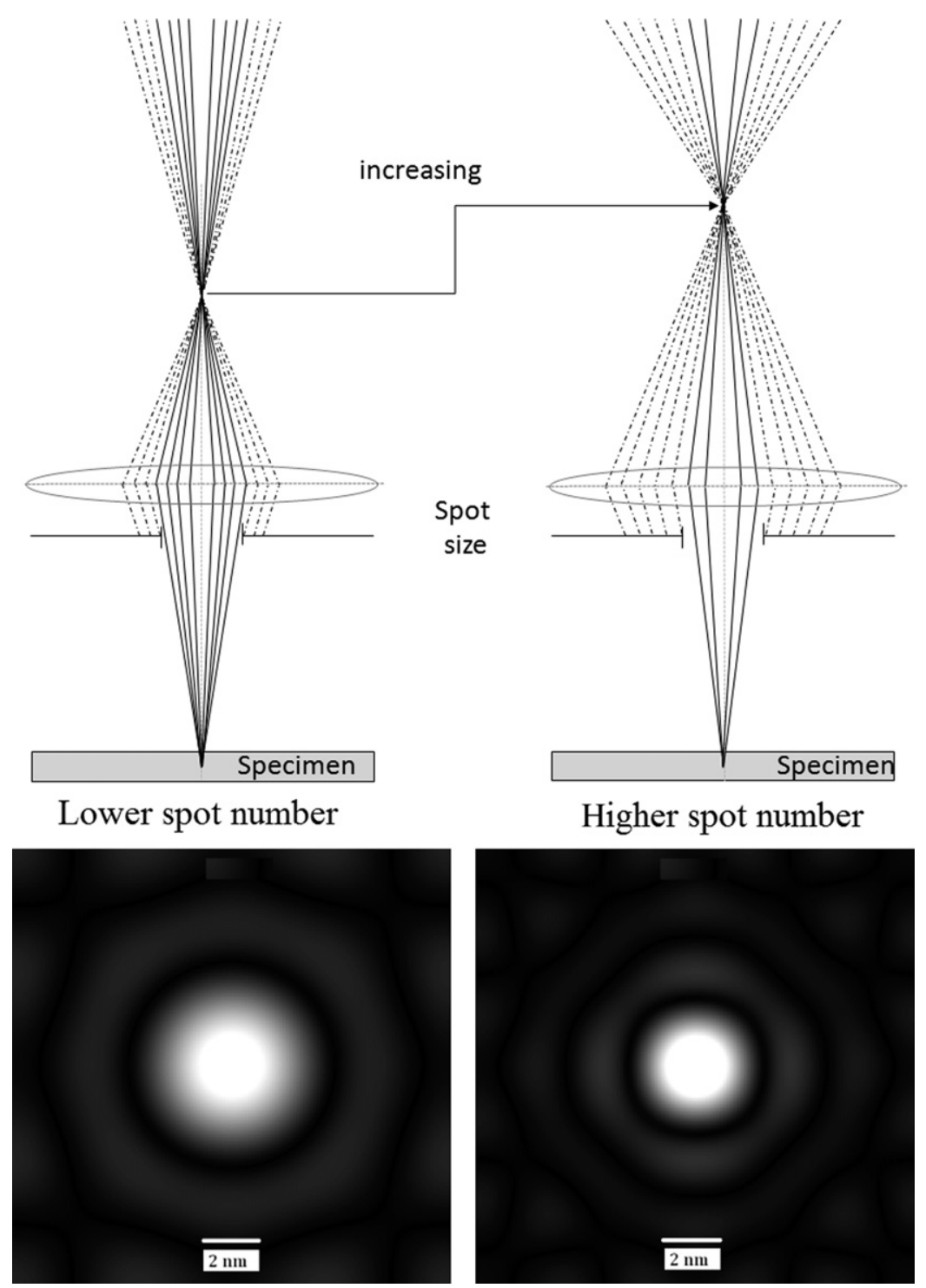

Figure 4.8 Electron beam sketches varying the spot size and the potential projected simulated depending on the spot size.

different sizes of the condenser apertures. The selected aperture must be centered in the ronchigram (described in next section) as shown in Figure 4.9.

Spot size and aperture of the condenser lens determine the resolution of the microscope in STEM mode. The spot size, measured in 

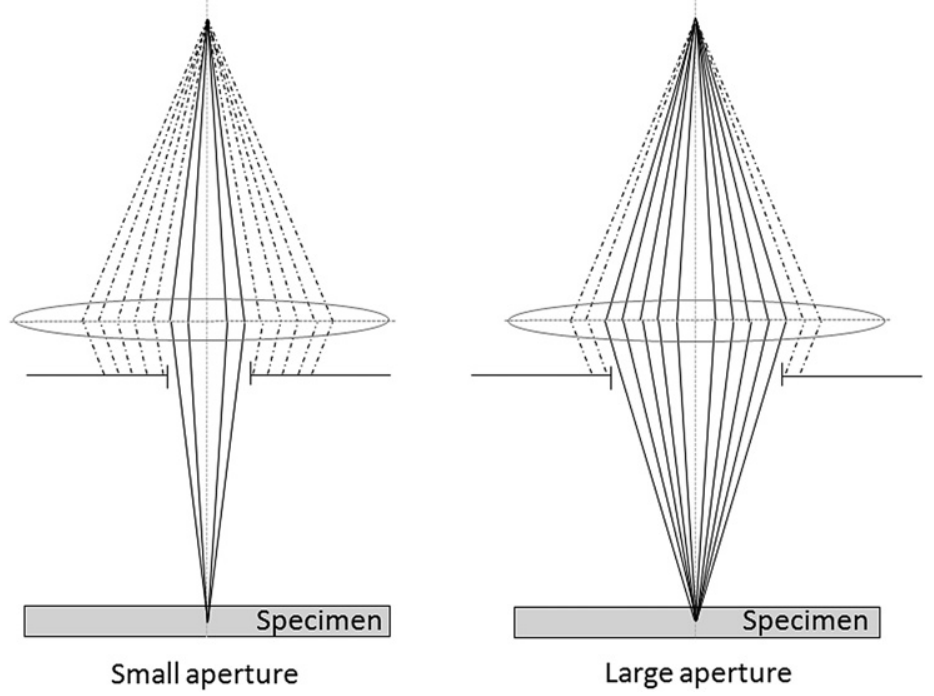

Figure 4.9 Electron beam trajectories by varying the condenser lens apertures.

nanometers, can be simulated as a function of the parameters of the microscope (Zuo, 2008).

A very useful tool is the ronchigram, which is a shadow image formed by a focused and stationary electron probe on amorphous material. The quality and resolution of the STEM images depend directly on the proper alignment of the ronchigram. It is easily observable in FEG microscopes, but it might be difficult to obtain when lanthanum hexaboride $\left(\mathrm{LaB}_{6}\right)$ filament sources are used because the effective probe size is too large; therefore both the spatial resolution and the brightness decrease dramatically even with microscopes with voltages exceeding $200 \mathrm{kV}$. All the parameters that affect contrast and spatial resolution also affect the ronchigram. The ronchigram can be registered in a charge-coupled device camera. The ronchigram is also known as the Gabor hologram, or as the central zero-order disk of the convergent beam electron diffraction (CBED) pattern. The ronchigram from an amorphous region should look as in Figures 4.10a and 4.10c. The shape of the ronchigram can be simulated using the parameters of the microscope (Zuo, 2008).

In the ronchigram, the focus spot must be represented in the center of the shadow image as a region of infinite magnification. Overfocus, in-focus, and underfocus ronchigrams and the beam incident on the sample are illustrated in Figure 4.11. 

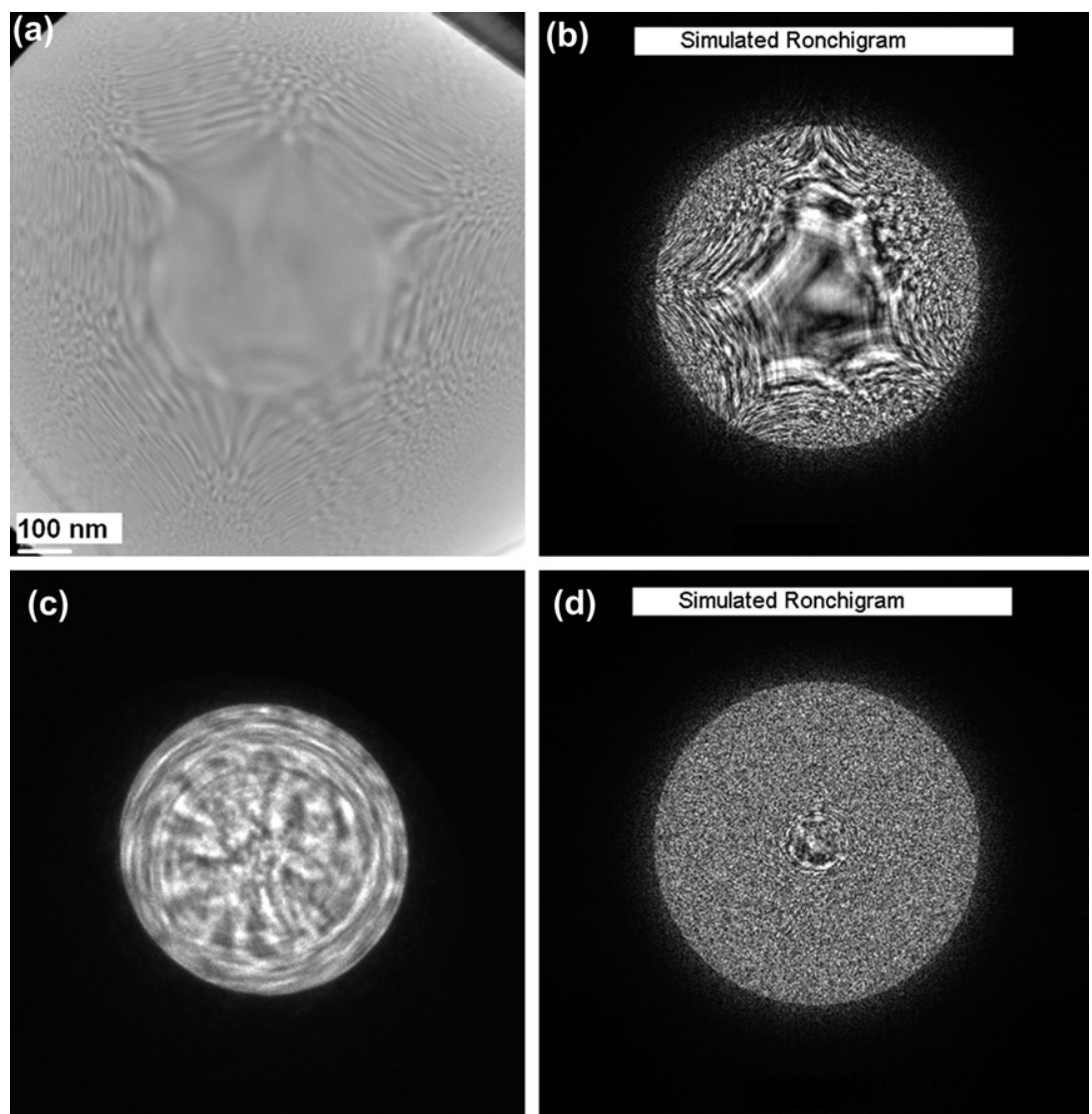

Figure 4.10 Aberration-corrected (a) experimental ronchigram from an amorphous material. (b) Calculated ronchigram. (c) aberration-uncorrected experimental ronchigram from an amorphous material. (d) Calculated ronchigram.

\section{EXAMPLES OF APPLICATIONS TO CATALYST CHARACTERIZATION}

\subsection{Metal Catalysts on a Crystalline Substrate}

For metal catalysts supported on a crystalline substrate, the best technique to observe the nanoparticles is STEM-HAADF, because with this technique the reflections of the metal and the substrate are clearly separated on the reciprocal space and it is straightforward to visualize the nanoparticles. A very common support for metal nanocatalysts is $\mathrm{TiO}_{2}$, which is very easy to grow and has been used extensively (Diebol, 2003). Figure 4.13a shows an example from the laboratory of Dr. Gabriela Diaz at the Institute of 


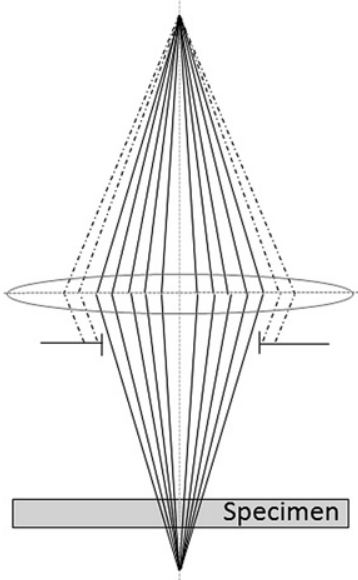

Underfocus

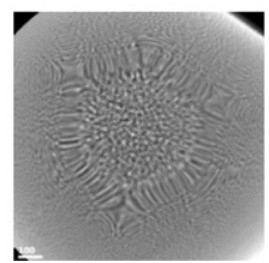

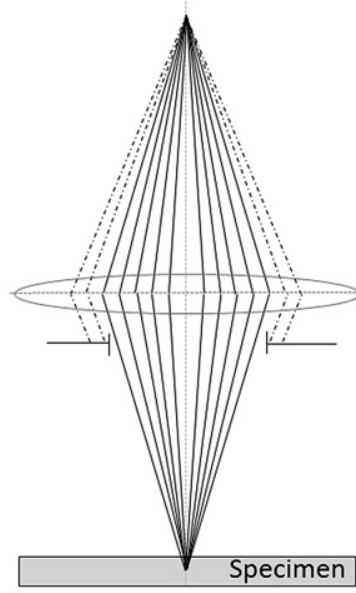

In-focus
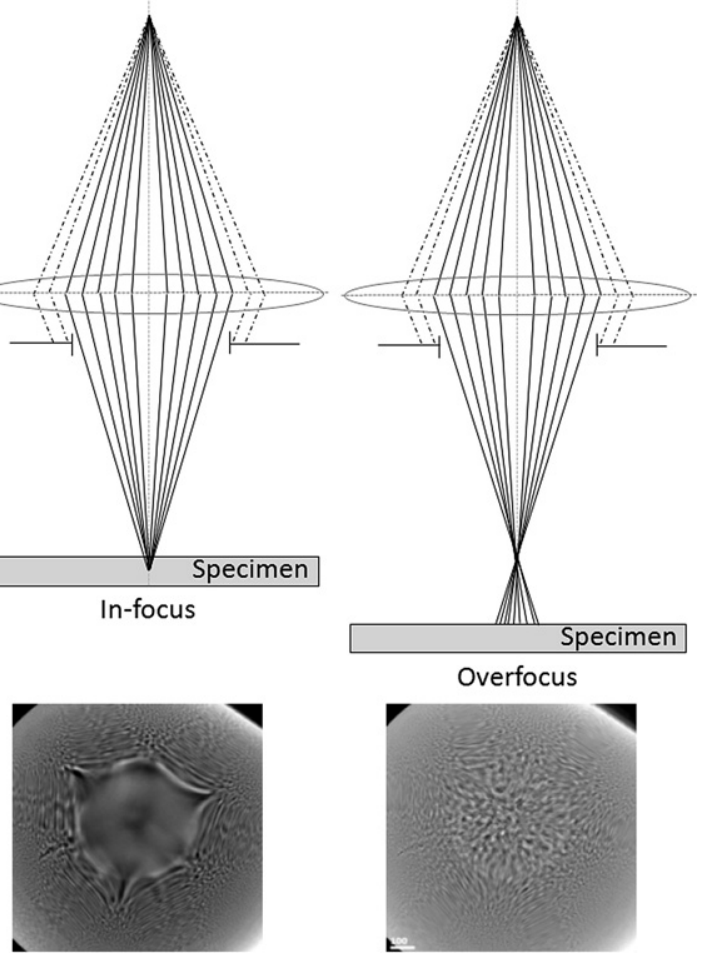

Figure 4.11 Ronchigram for different focus conditions.

Physics at the National Autonomous University of Mexico (UNAM). The micrograph corresponds to an iridium metal supported on a $\mathrm{TiO}_{2}$ (anatase) substrate. The metal clusters are clearly seen as formed white spots, each representing an atomic column. To further demonstrate the potential of the technique we can measure the intensity profile of the spots, and since there is only one metal on the sample, the intensity should be proportional to the number of atoms on the column. Figure $4.12 \mathrm{~b}$ shows the intensity profile for the cluster marked with an arrow in Figure 4.12a. The profile indicates that the four columns have the same number of atoms indicating a flat or "raft" shape (Jang et al., 2009). A further calibration of the intensity is required if the exact number of atoms is to be determined. In this case, we must first filter the substrate contribution and find a suitable region with isolated atoms. In any case, the relevant information for catalysis is that the atoms will expose a (100) facet to the reactant species. A cube-shaped iridium metal (Figure 4.12c) is not expected to be stable by itself but the presence of the crystalline surface makes it stable. 
(a)

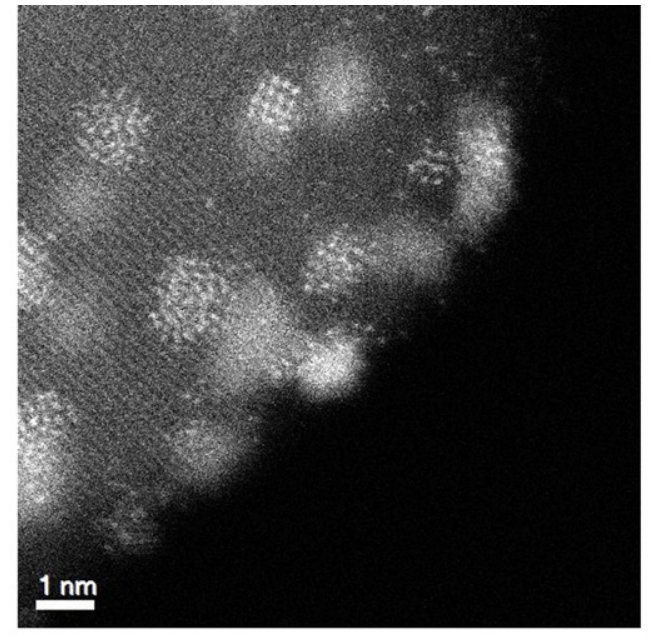

(b)

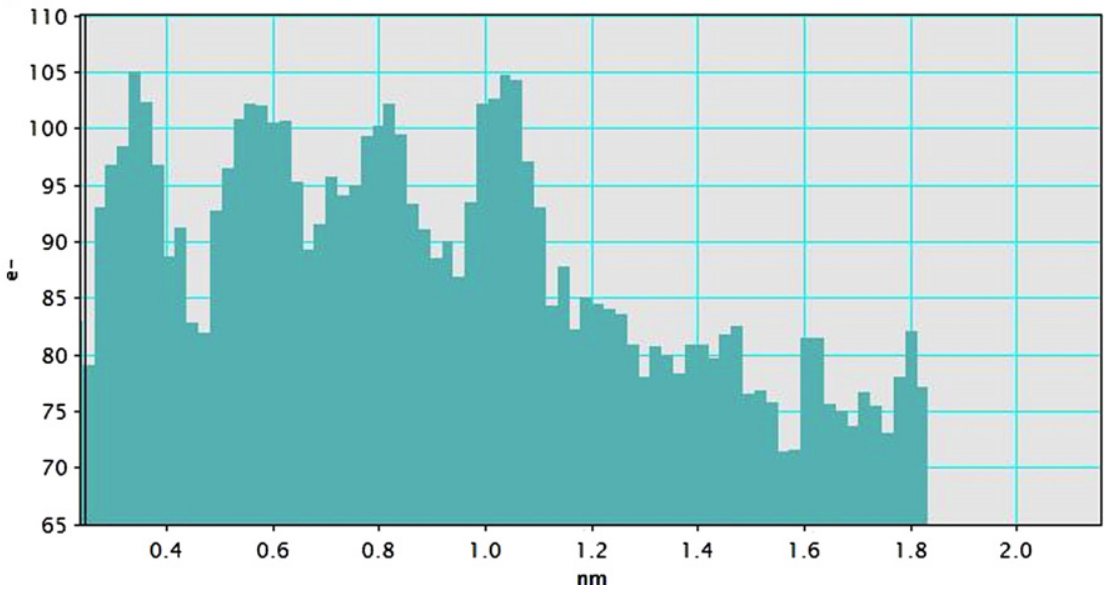

Figure 4.12 (a) STEM-HAADF image of an iridium supported on a $\mathrm{TiO}_{2}$ crystalline substrate. The clusters of metal are apparent. (b) Intensity profile of the atomic columns of the cluster marked with an arrow in (a). (See the color plate).

Figure 4.13 shows the dramatic difference between bright (STEM) and HAADF-STEM for an $\mathrm{Au} / \mathrm{TiO}_{2}$ catalyst. In the BF mode only the crystal structure of the catalyst can be observed. However, in the DF image not only clusters but also single atoms can be observed. Clearly, STEM-HAADF should be used when possible. The question that now arises is: Can some chemical information be obtained about clusters this size? The first point that should be very clear in this chapter is that while X-ray EDS and EELS 

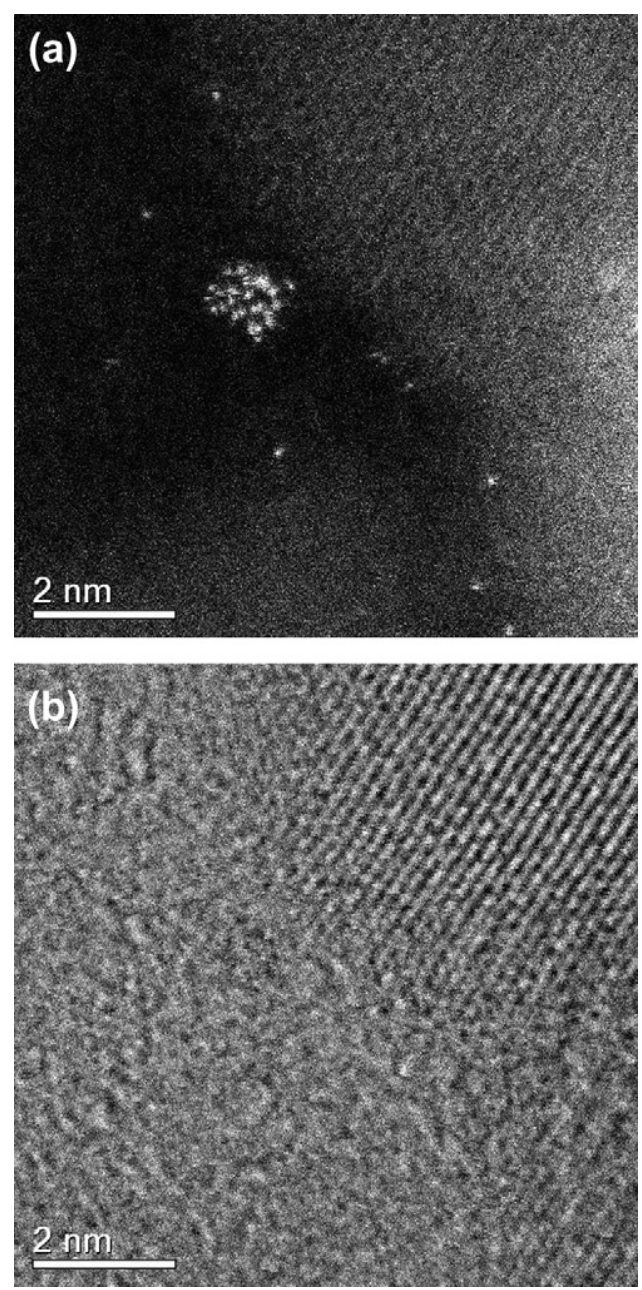

Figure 4.13 Images of an $\mathrm{Au} / \mathrm{TiO}_{2}$ catalyst showing the contrast of the two techniques: (a) HAADF and (b) BF-STEM of the same area.

(Friel and Lyman, 2006; Stroppa et al., 2012) are great techniques to accomplish chemical mapping and can be used in catalysis, as shown in other sections of this work, they are very limited for small clusters. Indeed, in many relevant cases the catalysts will be ultra-dispersed-that is, they will contain a few atoms or their size will be a few nanometers. This allows the catalyst to have a large number of atoms at the surface (or even in an ideal case, all the atoms in the surface). However, the number of $\mathrm{X}$-rays emitted by the clusters during a microanalysis study will be low and the signal-to-noise ratio will be unacceptable. Often a catalyst researcher not fully familiar with the 
TEM technique expects the chemical composition of its clusters to be determined atom by atom. This certainly can be done in larger samples such as thin films or grain boundaries and even in nanoparticles larger than $5 \mathrm{~nm}$. However, it is not possible for particles $<5 \mathrm{~nm}$.

The same situation applies to EELS analysis. Is this the end of the road for chemical identification in very small clusters? The answer is no. STEM-HAADF can yield important information. Figure 4.14 shows an

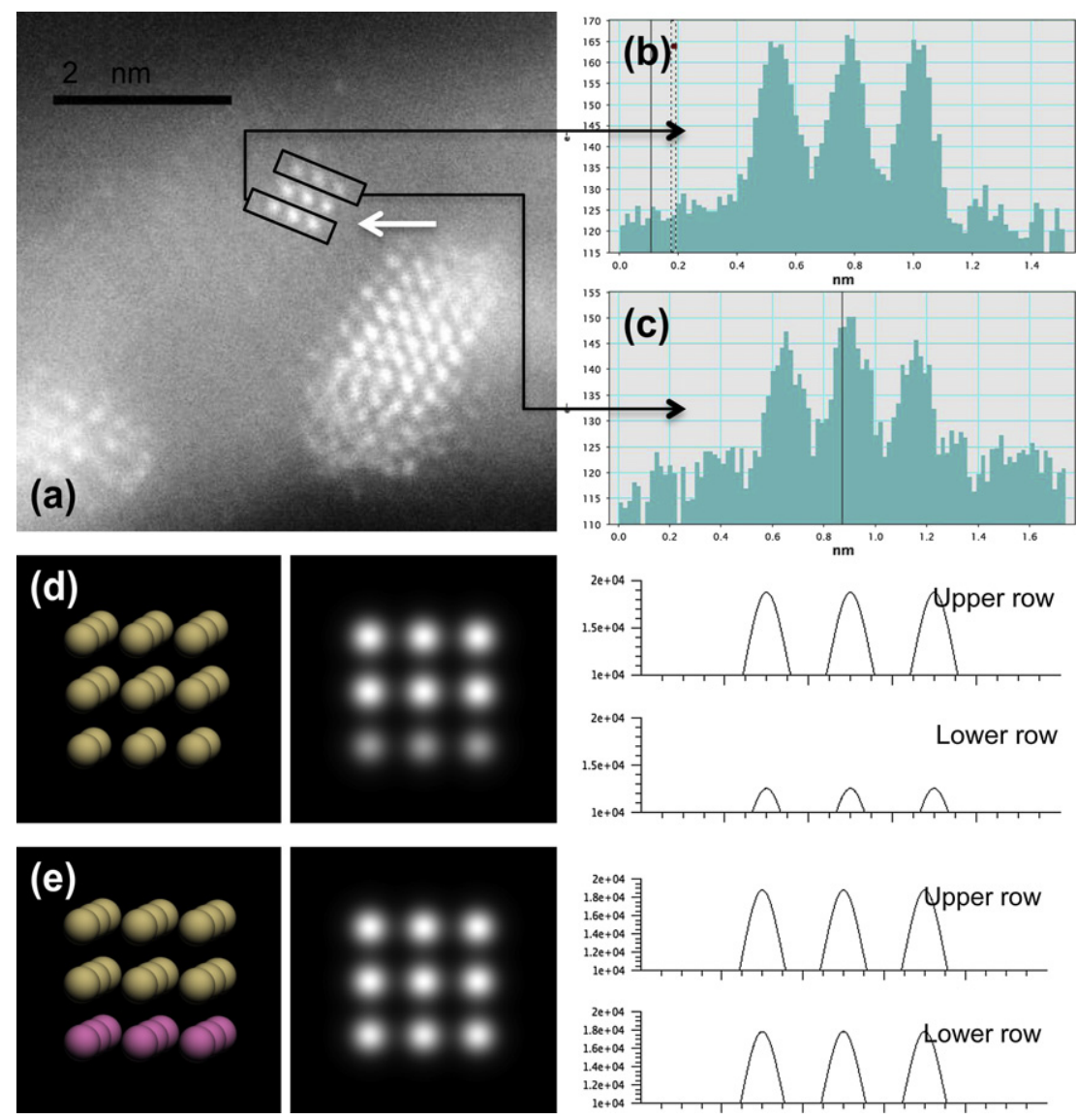

Figure 4.14 (a) STEM-HAADF Image of a Au/lr alloy supported in $\mathrm{TiO}_{2}$. The cluster marked with an arrow formed by $3 \times 3$ columns of atoms is analyzed. (b) Intensity profile of the first (bottom) row. (c) Profile of the third (top) row. Two possible models that are consistent with the data: (d) a stepped structure and (e) the Janus or "eutectic" type of structure. On the right, the simulated STEM micrographs and intensity profiles (arbitrary units) for the models in (d) and (e) are also shown. (See the color plate). 
example of a gold-iridium alloy supported on $\mathrm{TiO}_{2}$. Figure 4.14a shows the HAADF-STEM image of these clusters. If we analyze the rectangular cluster marked by a white arrow, we can obtain the signal intensity for each column of atoms. Figure $4.14 \mathrm{~b}$ shows the profile for the row of atoms at the bottom. The profile for the next row is similar to the one of Figure 4.14b. However, the row at the top has the same profile but with a lower value of the intensity shown in Figure $4.14 \mathrm{c}$. There are two possible explanations. The first is that all the atomic columns are thicker than one monolayer and all atoms have the same atomic number. In that case, the decrease in intensity would have to be explained as a step on the structure (as shown in Figure 4.14d). The other situation can be obtained by noting that the ratio of the intensities is 0.94 and if we assume that the last column is iridium and the first two are gold, then the ratio of the STEM signals according equations described before is 0.94. Therefore, a very plausible model is the one shown in Figure $4.14 \mathrm{e}$ in which one row corresponds to iridium atoms and two rows correspond to gold atoms. This is the Janus-type particle. Of course, this is not a definite proof and additional data are required. A careful calibration of intensities for a more involved model can be made to obtain intensities using the methods described in Kotaka (2010) and Recnik (2005).

\subsection{Metals on a Noncrystalline Substrate}

A second relevant case concerns particles supported in an amorphous or semi-amorphous substrate. In this case, the contrast is more complicated since the substrate also has incoherent electrons scattered at high angles, thus increasing the signal-to-noise ratio. Therefore, a more careful interpretation is needed. A typical case is that of the metals supported on Vulcan carbon. Figure 4.15 shows such an example for iridium. Clearly, the HAADF-STEM image can be used to very accurately determine the particle size in large areas (and the particle size distribution). The fact that no delocalization effect, which is very pronounced on field emission microscopes, is present on this type of images (Carter and Williams, 2009) makes the size determination very accurate.

In some special cases, the Z-contrast can be nicely combined with BF images to complete the picture. Figure 4.16 shows an example in which a platinum nanoparticle is supported in a carbon fullerene onion (CNO). The images, both BF and DF, allow the location of particle on the graphitic structure. It is clear that the particle is located in the interlayer zone of the 

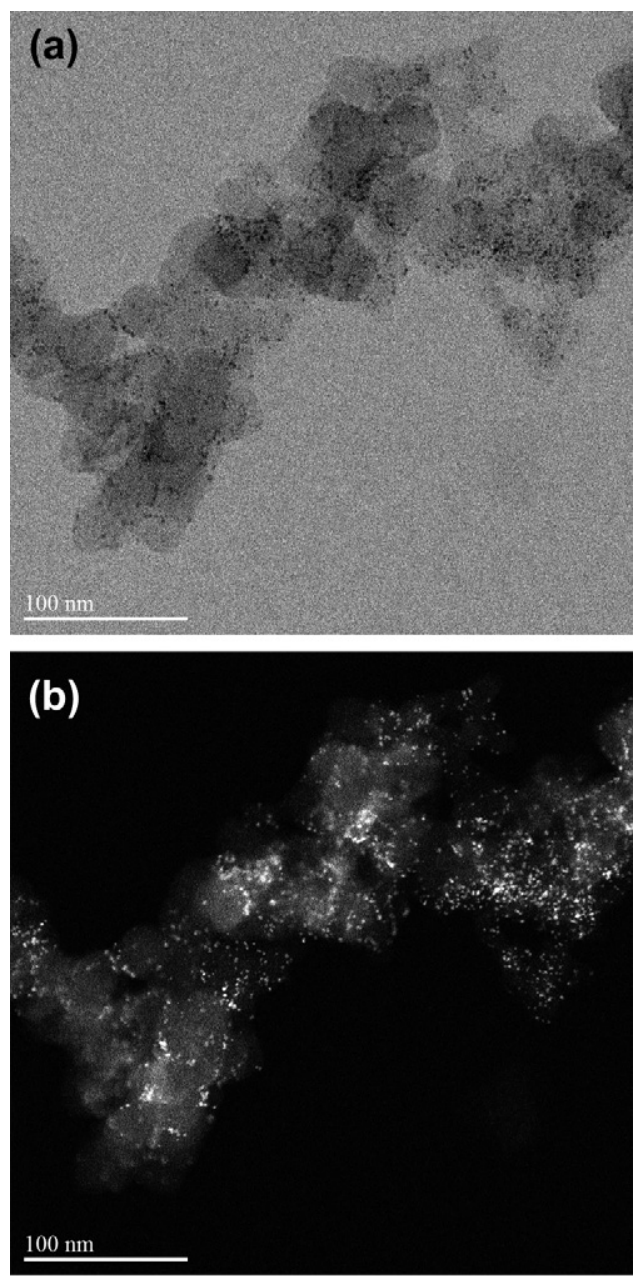

Figure 4.15 Example of contrast of iridium supported on Vulcan carbon. The images of the particles in STEM-HAADF are ideal for size distribution determination. (a) DF- and (b) BF-STEM bright field.

onion (Figure 4.16b). In this case, the background from the substrate acted in favor of a more complete characterization.

In some cases, amorphous carbon produces too much noise on the Z-contrast images, and often it is better to use HREM TEM in the transmission mode. This is more important when the catalysts are ultradispersed and the nanoparticles contain very few atoms. Figure 4.17 shows an example for a palladium catalyst supported on Vulcan carbon. 

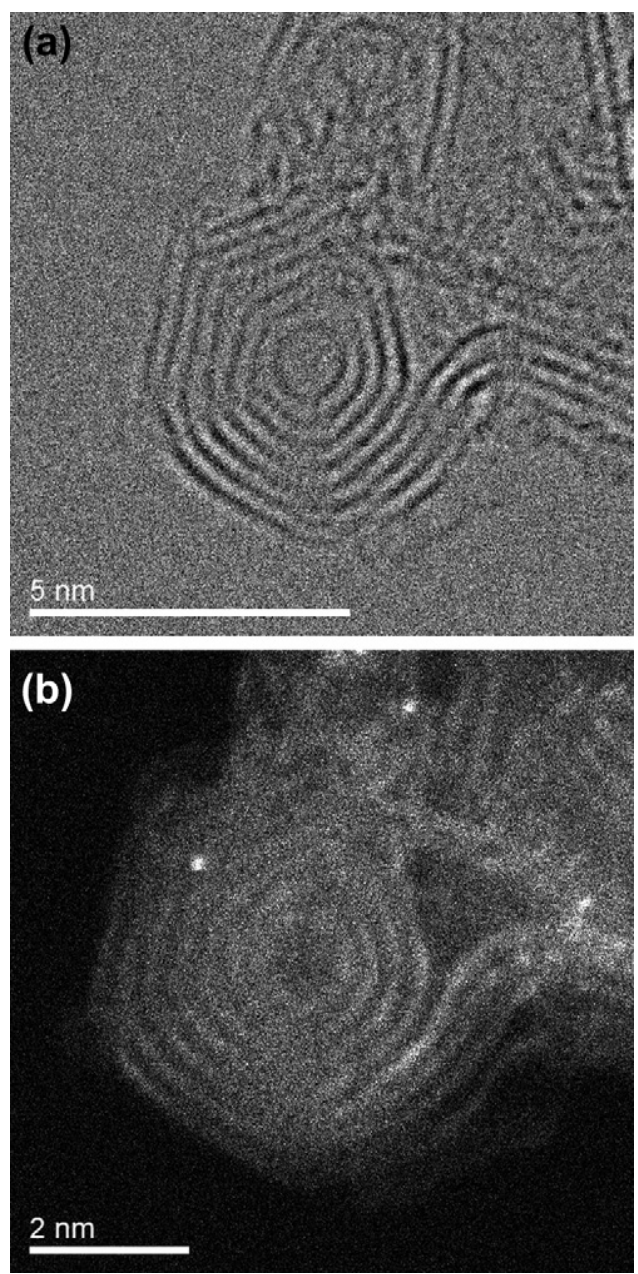

Figure 4.16 Pair of STEM-HAADF images. (a) DF and (b) BF of a CNO onion structure with a platinum particle.

In this case, the aberration-corrected image show clusters consisting of a few atoms. There is no doubt that BF-TEM works much better for this particular case.

\subsection{Metals on $\gamma-\mathrm{Al}_{2} \mathrm{O}_{2}$}

Some of the most important catalysts on petroleum refining are noble metals such as platinum, palladium, ruthenium, rhodium, and recently even gold supported on $\gamma-\mathrm{Al}_{2} \mathrm{O}_{3}$ (Frenkel et al., 2001; Rioux, 2006; Ahmed et al., 2009). The alumina is a porous support with a face-centered cubic (FCC) 


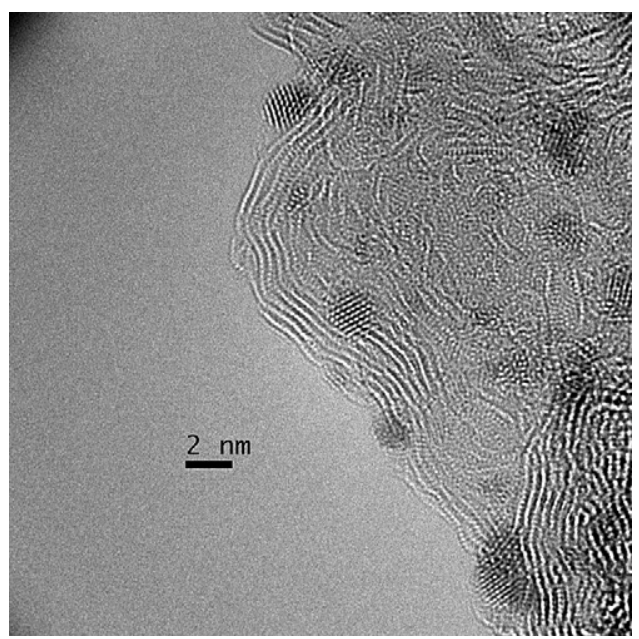

Figure 4.17 HR-TEM image using an aberration corrected TEM of palladium nanoparticles supported on Vulcan carbon. Atomic resolution of the clusters can be clearly observed. In this case, the signal-to-noise ratio is very good to distinguish nanoparticles from the support.

structure $a=7.90 \AA$. The structure of the metal has been the subject of vast investigation (Ahmed et al., 2009). The point is whether the cluster is flat (rafts or 3D in shape). Aberration-corrected STEM-HAADF can offer clues. Figure $4.18 \mathrm{a}$ shows an image of a $\mathrm{Pt} / \gamma-\mathrm{Al}_{2} \mathrm{O}_{3}$ catalyst. The profile of the cluster marked with $\mathrm{A}$ is shown in Figure $4.18 \mathrm{~b}$. The particle is clearly 3D because of the Gaussian shape, and its size is about $1 \mathrm{~nm}$. One structure consistent with this size and shape is the on 13-atom icosahedron. The diffraction from these planes falls in reciprocal space very close to the highangle scattering of the metal. Therefore, it is possible to see single atoms intercalated on the planes. If we calibrate the intensity of a single atom in the same figure and consider the ratio with the maximum intensity of the cluster, we find that $N$ (the number of atoms in the cluster) is 2.8 for the maximum value of intensity; this is again consistent with the icosahedral shape. So it appears that the idea of rafts is not valid in this case, but the catalyst seems to have most of the atoms on the surface. A 13-atom icosahedron is a possibility. However, a new important possibility for metals was recently found by Longo and Gallego (2006), who reported that for platinum a buckled planar structure is more stable than the icosahedron. This structure, which also has 13 atoms, is shown in Figure 18c and will have all its atoms on the surface. This appears quite consistent with the experimental 
(a)

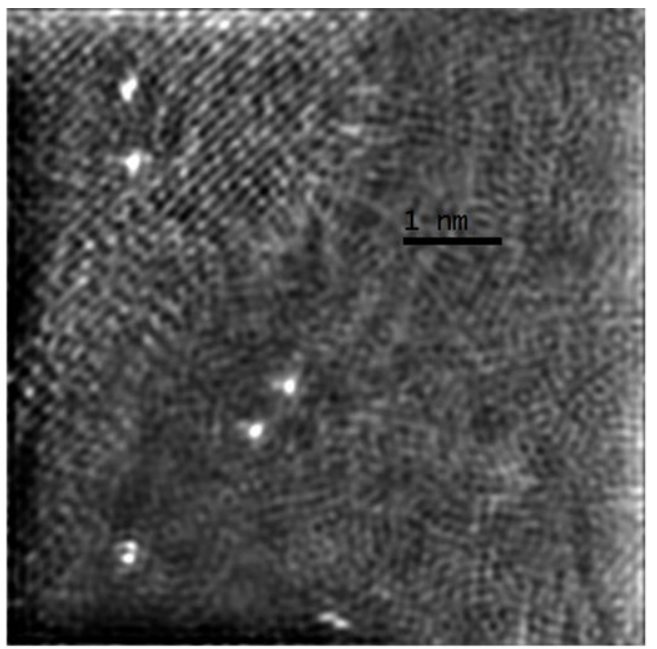

(b)

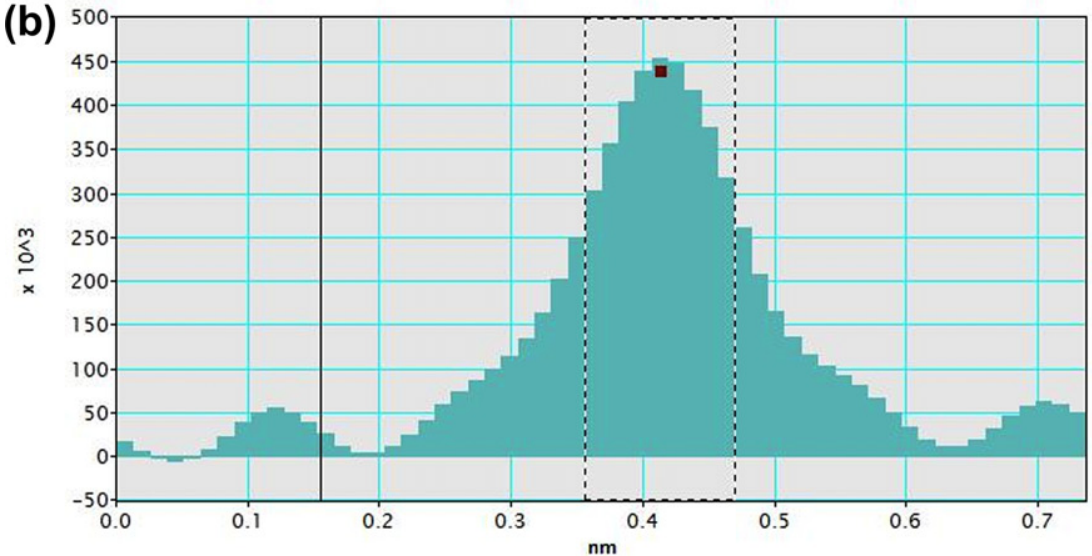

(c)

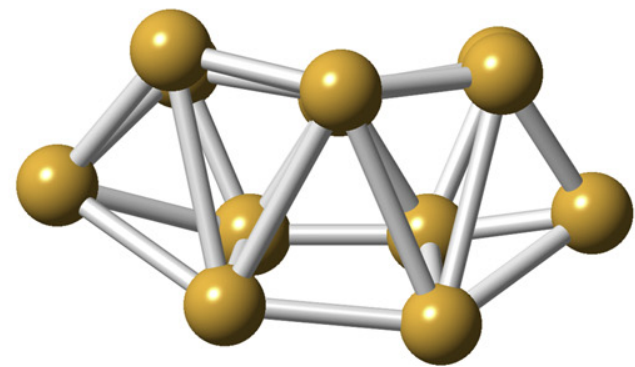

Figure 4.18 (a) TEM-HAADF image of a Pt/ $\gamma-\mathrm{Al}_{2} \mathrm{O}_{3}$ catalyst. The (444) lattice lines of the alumina can be observed. The bright dots correspond to clusters and to single atoms. (b) Intensity profile of the cluster marked with an arrow in a 3D shape is not consistent with the structure of a raft. (c) A 13-atom optimized cluster is shown that might correspond to the shape observed. (For color version of this figure, the reader is referred to the online version of this book). 
observations since the intensity profile will be consistent with two layers. The other platinum metal on the catalysts will be present as individual atoms.

A conclusion from the previous discussion is that flat or raft types of structures seem to be stable only when there is a strong metal-substrate interaction as in the case of metal- $\mathrm{TiO}_{2}$. In the case of weakly interacting substrates such as $\gamma-\mathrm{Al}_{2} \mathrm{O}_{3}$, pseudo-spherical shapes are preferred.

\subsection{Bimetallic Nanoparticles and Clusters}

Bimetallic nanoparticles are a combination of two individual metallic systems that come together to form an alloy. This combination serves as a platform to enhance both their individual and collective properties. Thus, compared with monometallic systems, bimetallic systems have greatly improved properties; a typical case, for example, is their improved reactivity in catalysis for many varied reactions. For bimetallic nanoparticle alloys, the synergy in the hybrid material can presumably be attributed to the electronic coupling of the individual metals and in addition to the geometric effects that may be caused by the different lattice constants (Stamenkovic et al., 2007). The concept of bimetallic nanoparticles goes back to the 1970s where it was used in catalysis of two different metals such as gold and palladium in the same nanoparticle (Sinfelt, 1983). This idea was developed by Toshima's group, (Harada et al., 1993) who used polyvinylpyrrolidone (PVP) to stabilize core-shell bimetallic gold-palladium nanoparticles-for instance, nanoparticles in which the core is gold but palladium atoms are located on the shell $(\mathrm{He}$ et al., 2003). Subsequent to coreduction, this structure is controlled by the order of reduction potentials of both ions and the coordination abilities of both atoms to PVP. The location of gold in the core and palladium on the shell was demonstrated by extended X-ray absorption fine structure, and it was shown that such heterobimetallic gold-core palladium nanoparticles are more active in catalysis than simple PVP-stabilized palladium nanoparticles. Thus, the gold core enhances the catalytic properties of palladium nanoparticles at the palladium nanoparticle surface (Toshima, 1996). Conversely, design strategies can lead to the opposite core-shell structure (palladium core, gold shell), and specific catalytic properties were obtained for methylacrylate hydrogenation (He et al., 2003). Thus, with the observation of a remarkable enhancement in the properties of bimetallic nanoparticles, they have continued to evoke vigorous scientific interest. 


\subsection{Understanding the Structure of Bimetallic Nanoparticles and Clusters Using Advanced Electron Microscopic Techniques}

Direct microscopic studies of various bimetallic nanoparticles have been performed using HR-TEM (Perez-Tijerina et al., 2008; Perez-Tijerinam et al., 2010; Mejia-Rosales et al., 2006). However, this method is limited because of the small difference in the lattice constant involved. TEM can produce chemical contrast due to different electron beam extinction distances. This has been applied successfully to image bimetallic nanoparticles down to $10 \mathrm{~nm}$ in diameter. Since the particles need to be in a low-index zone orientation, this method is not easy to use in practice (Srnová-Šloufová et al., 2000). A novel approach to study this type of particles is based on the use of an HAADF technique in a TEM, which allows the observation of the interfaces between layers of different elements due to differences in atomic number, densities, or the presence of strain fields due to differences in lattice parameters. However, in recent times the study of bimetallic nanoparticles has been greatly improved with the use of aberration-corrected scanning/transmission electron microscopy (S/TEM), which yields a new level of understanding of the behavior of these systems at the atomic scale. A combination of the HAADF imaging mode in an aberration-corrected STEM/TEM reveals a wealth of information about these systems. It is well established that, when recorded under appropriate illumination and collection geometries, incoherent HAADF-STEM images are compositionally sensitive and provide direct information on atomic positions, which is crucial in the differentiation of variation in the atomic compositions while imaging.

The conventional understanding of bimetallic nanoparticles is that they have one of four fundamental structures: (1) alloys, (2) core-shell, (c) onion, or (d) Janus (Figure 4.19).

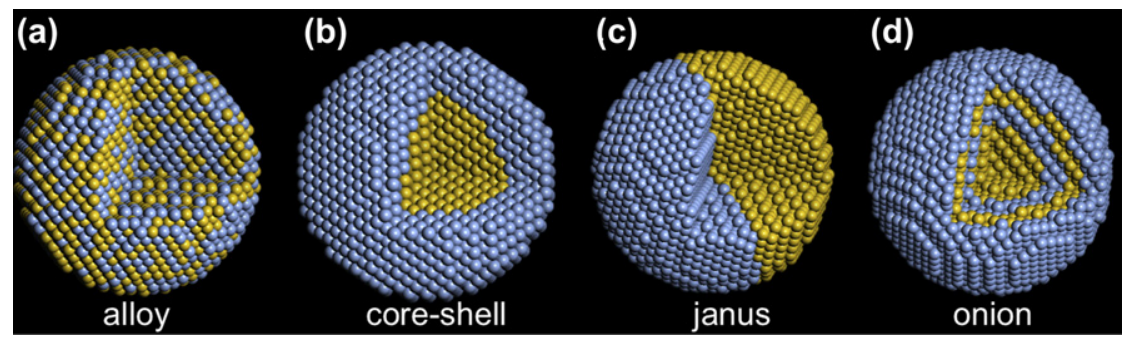

Figure 4.19 The four main types of structures on bimetallic nanoparticles: (a) alloys, (b) core-shell, (c) Janus structure, or (d) onion structure. (See the color plate). 
Several factors play a role in determining which of the four alternatives is more likely to occur. These factors include the solubility of the metals, the relative surface energy, the kinetics of the synthesis, and several others. In the case of gold-palladium nanoparticles, both types of structures can be formed. Gold-palladium is a particularly interesting system because of its applications as hydrogenation catalysts and the two metals are fully soluble in bulk. Hence, both alloy nanoparticles, as well as core-shell gold-palladium nanoparticles, can be obtained depending on how the individual metal ions themselves are reduced (Mizukoshi et al., 2000). Figure 4.20 shows the probe aberration HAADF images of nanoparticles of $\mathrm{AuPd}_{3}$ with the alloyed structure. It is clear that some atomic columns are significantly brighter that adjacent columns, as shown by the contrast of the atom row with arrows in the inset of Figure 4.20b. The top of Figure 4.20c shows intensity traces over two single atoms labeled atom 1 and atom 2 . These are typical of several such traces in Figure $4.20 \mathrm{~b}$ and suggest that the higher-intensity atom is gold and the lower one is palladium. Since the intensity is proportional to $Z^{1.6}$, the relative single-atom intensities should be 791.6/461.6 $=1087 / 458=2.37$. It is very reasonable to assume that the more-intense atoms correspond to gold and the less intense atoms correspond to palladium since the intensity ratio is 1.8 , a reasonable approximation of the expected ratio of 2.37 , given the low sampling and low signal above background. With regard to the bright central column, if it is all palladium, then it represents an unreasonable number (at least three) of the palladium atoms extending above the general
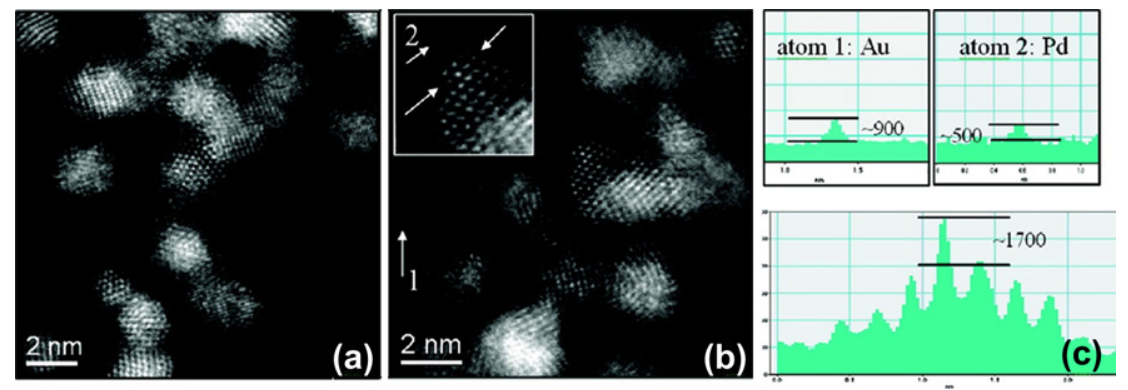

Figure 4.20 (a) and (b) HAADF images of typical $\mathrm{AuPd}_{3}$ nanoparticles. Single-atom traces (top of (c)) suggest a palladium intensity of 500 counts versus a gold intensity of 900 counts. The profile of the atom row indicated on the inset (c, bottom) shows a central column roughly triple the intensity of the adjacent column, presuming all palladium atoms. This suggests the presence of one or more gold atoms in the central column. (Reprinted with permission from Mejia-Rosales et al. (2006). Copyright (2007) American Chemical Society). (See the color plate). 
surface. This is not favored energetically; it is likely therefore that one or more gold atoms contribute to the intensity of this column. The roughness of the surface, due to the presence of extended point defects, can also be seen clearly.

A different situation can be seen for gold-palladium nanoparticles which were obtained by successive reduction of metal ions. In this case it was found that when particles (with different gold and palladium compositions) were synthesized by chemical methods, a much more complex situation arises, in which the nanoparticles show a structure with three alloyed layers and with different compositions (Ferrer et al., 2008). Figure 4.21 shows the ultra-high-resolution HAADF images of the gold-palladium nanoparticles obtained in a probe aberration-corrected STEM. From Figure 4.21a it can be seen that the three layers in the nanoparticle are clearly evident. The diameter of the nanoparticles is $\sim 8 \mathrm{~nm}$. This consists of a palladium core (A $\sim 2 \mathrm{~nm}$ ), gold-rich first shell (B $\sim 3 \mathrm{~nm}$ ), and palladium-rich outer shell $(\mathrm{C} \sim 3 \mathrm{~nm}$ ) (shown from the contrasts in the HAADF images in Figure 4.21a). The HAADF intensity is considered to be proportional to $Z^{1.6}$ and hence the difference in atomic number $(Z)$ that exists between gold and palladium is clearly revealed in the three-layer nanoparticles (gold, $\mathrm{Z}=79$; palladium, $\mathrm{Z}=46$. The shape of this particle corresponds to

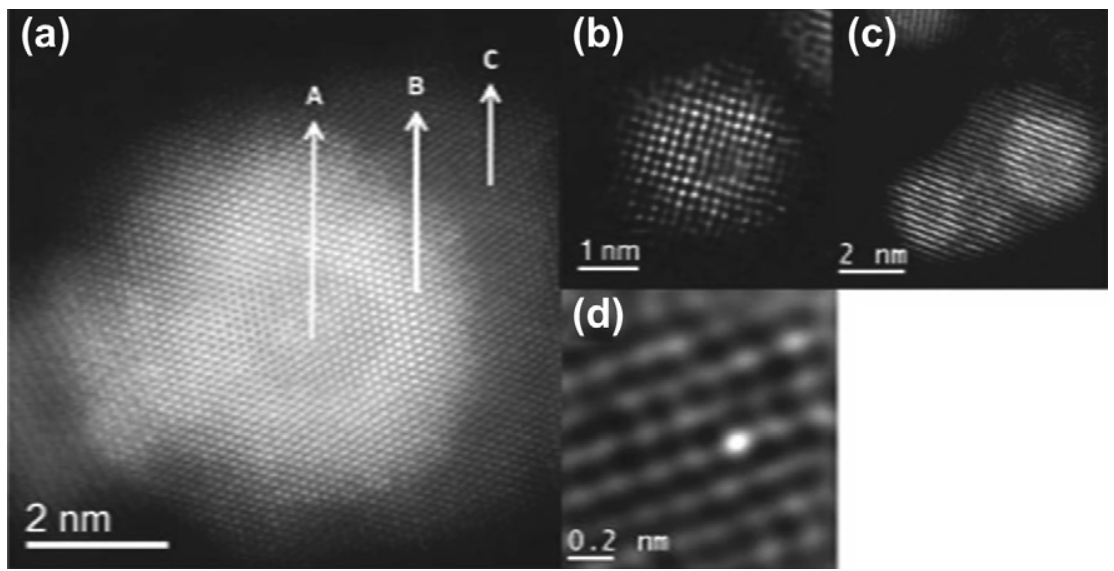

Figure 4.21 Aberration-corrected STEM images of the three-layer Au/Pd nanoparticles. (a) Atomic-resolution HAADF-STEM image of a truncated cuboctahedral Au/Pd nanoparticle. The contrast of the three distinct regions (marked A (palladium), B (gold), and C (palladium)) can be clearly identified. (b) Nanoparticles with rough surface and distortions at the core. (c) and (d) Gold intercalation into the palladium layer. (Reprinted with permission from Deepak et al. (2011a). Copyright (2011) Elsevier). 
a truncated octahedron, which has (111) and (100) facets. Figure 21b shows a three-layer particle with the (100) orientation where roughness on the surface and distortion in the core of the nanoparticle can be seen. In some nanoparticles, it may also be seen that the gold atoms are indeed diffused into the palladium-rich outermost shell as seen from the bright atom contrast in the palladium layer. Figures $21 \mathrm{c}$ and $21 \mathrm{~d}$ show the atom-by-atom intercalation of gold in the palladium layer. This shows how gold can be present in the palladium layers and is not entirely rigid. So the layers indeed are alloys of different concentrations (Deepak et al., 2011a).

The energy dispersive analysis of X-ray (EDAX) for the three-layer goldpalladium-gold nanoparticles reveals the following interesting features (Figure 4.22a). The palladium L-shell and the gold L- and M-shell signals can be clearly be used to identify the individual palladium and gold layers (Figure 4.22b). Figure 4.23 shows the chemical maps of the gold-palladium nanoparticles. It can be seen from the figure that the maps for the palladium ( $\mathrm{K}$ and L shells) and gold ( $\mathrm{L}$ and $\mathrm{M}$ shells) clearly reveal the presence of the individual elemental contrasts in the three-layer core-shell structure of the nanoparticles. With the information obtained from the EDAX line scans and elemental maps of the individual nanoparticles, the presence of the palladium core, gold first shell, and palladium outer shell is clearly confirmed without any ambiguity.

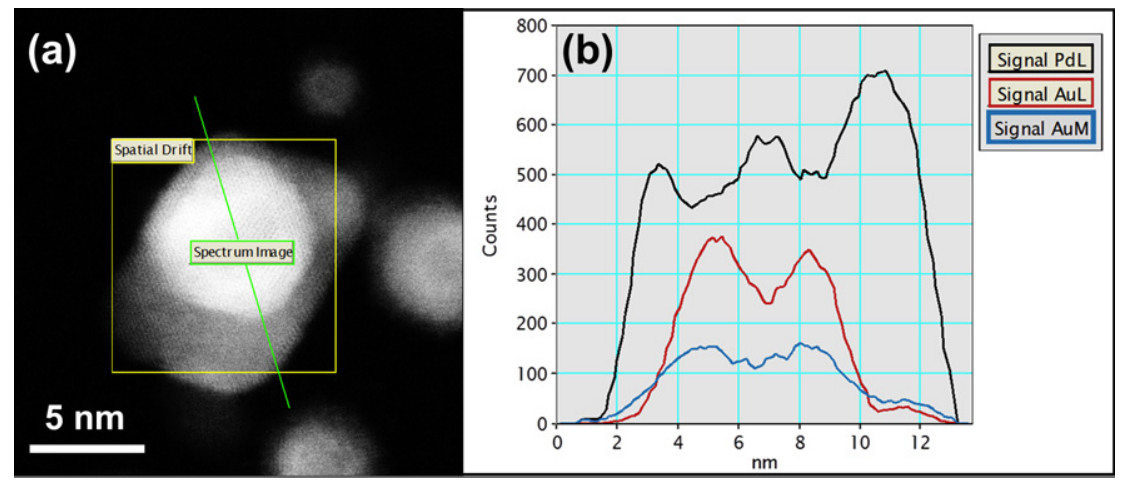

Figure 4.22 (a) and (b) Characterization of the gold and palladium elemental distribution across the nanoparticle by STEM-EDAX line-scanning technique across the individual three-layer nanoparticle. The Pd-L and the Au-L,M line scan signals (b) can be clearly seen varying in intensity along the different regions of the nanoparticles. (a) The analyzed area and the direction of analysis. (Reprinted with permission from Deepak et al. (2011a). Copyright (2011) Elsevier). (See the color plate). 

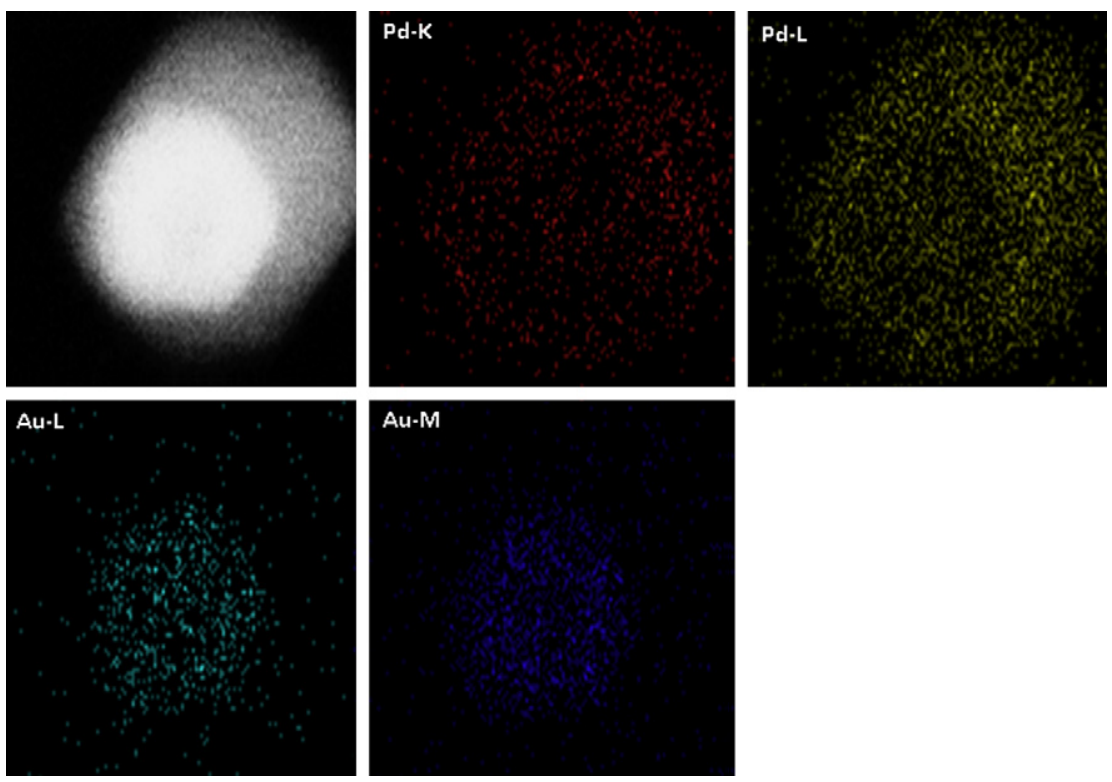

Figure 4.23 Elemental maps of the nanoparticles for Pd-K and Pd-L (top) and Au-L and Au-M (bottom). (Reprinted with permission from Deepak et al. (2011a). Copyright (2011) Elsevier). (See the color plate).

The distribution of the chemical elements of the three-layer nanoparticle (Figure 4.24a) was investigated by EELS; Figure 4.25b shows the result for the EELS line profile of $\mathrm{Pd}-\mathrm{M}_{4,5}$. For purposes of comparison, the figure also shows the HAADF intensity line profile of the same particle (Figure 4.24d). From the EELS profile (Figure 4.24c) it can be noted that the distribution of palladium appears to be in accordance with the presence of palladium in the core and in the outermost shell as is the case for a trilayer particle. However, the EELS Au-M $\mathrm{M}_{4,5}$ signal at $2206 \mathrm{eV}$ turns out to be weak, as expected for a heavy element, but high enough to show the presence of gold in the particle.

From the comparison between the palladium EELS profile and the HAADF profile, it can be inferred that a palladium-rich region exists close to the center of the particle, and another in the outermost shell. The corresponding EELS map of palladium clearly reveals the presence of palladium in the core and the outermost shell of the tri-layered nanoparticles (Figure 4.25).

It is important to note that in case of these nanoparticles a four-layered structure is seen occasionally. Figure 4.26 shows such a four-layer structure 

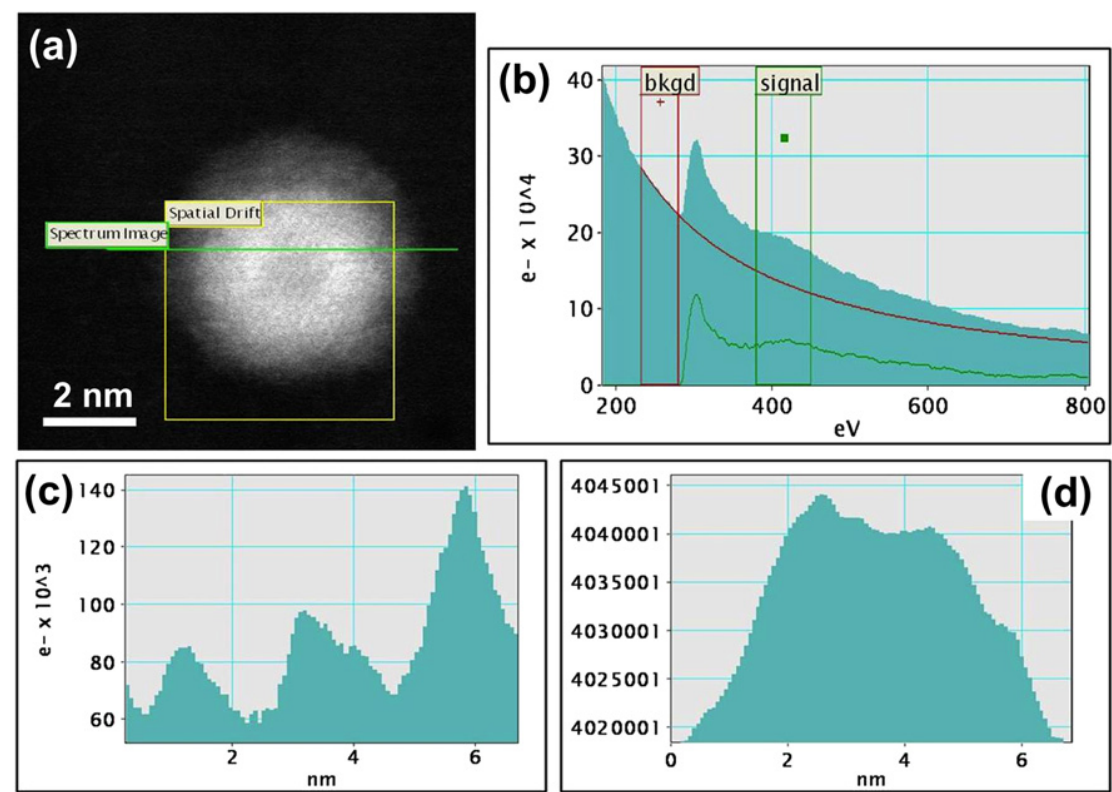

Figure 4.24 The distribution of the chemical elements of the three-layer nanoparticles. (a) Investigated by EELS; (b) the result for the EELS line profile of Pd-M $\mathrm{M}_{4,5}$. (c) Linear palladium EELS profile performed on the particles; (d) its corresponding HAADF intensity profile. (Reprinted with permission from Deepak et al. (2011a). Copyright (2011) Elsevier). (See the color plate).
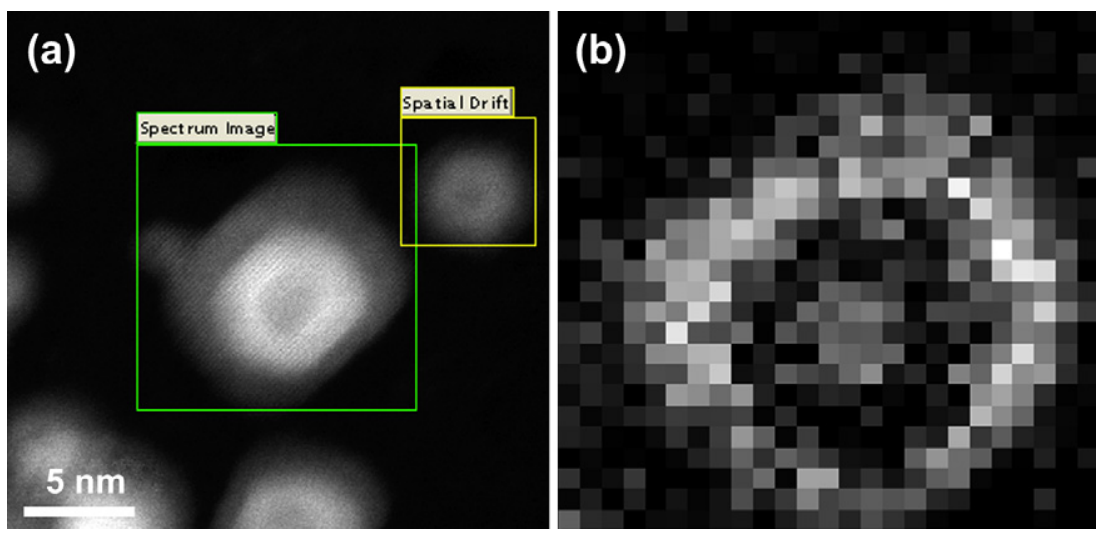

Figure 4.25 EELS map of palladium clearly reveals its presence in the core and in the outermost shell of the trilayered nanoparticles. (Reprinted with permission from Deepak et al. (2011a). Copyright (2011) Elsevier). (For color version of this figure, the reader is referred to the online version of this book). 


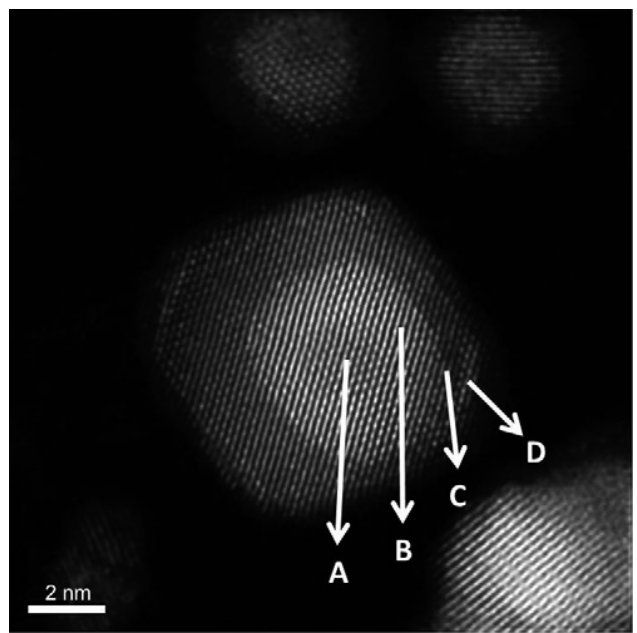

Figure 4.26 Aberration-corrected STEM images of the four-layer Au/Pd nanoparticles. The contrast of the four distinct regions (marked A, B, C, and D) can be clearly seen. (Reprinted with permission from Deepak et al. (2011a). Copyright (2011) Elsevier).

where an extra layer of gold atoms can be seen tnear the surface, which suggests the four layers. This is a different case from the previously mentioned three-layered nanoparticles and has not previously been reported.

The three-layered nanoparticles were investigated individually using nanobeam electron diffraction (NBD). This technique allows investigating the crystal structure in an area down to the nano scale by using a concentrated yet still nearly parallel electron beam with the probe size as small as a few nanometers or less (Ferrer et al., 2009). The analysis of the crystal structure using TEM NBD and a typical NBD pattern from a nanoparticle are shown in Figure 4.27a. This NBD pattern can be indexed using the hexagonal closepacked (HCP) structure. The NBD is oriented in the zone axis [21.1]. The experimentally (and theoretically) calculated values of the diffraction spots along with their respective angles are shown in Tables 1 and 2 . The lattice parameters calculated using the HCP structure are $a=b=0.4105 \mathrm{~nm}$ and $c=0.6647 \mathrm{~nm} \quad(c / a=1.619)$. The palladium-gold-palladium nanoparticles were analyzed by using weak-beam dark-field (WBDF) technique, which allowed obtaining topological information of the nanoparticles. Figure $4.27 \mathrm{~b}$ shows a WBDF image of a palladium-gold-palladium nanoparticle. The width and separation of thickness fringes is directly related to the angle of incidence between the electron beam and the sample. The thickness fringes produced by the stacking fault can be observed, which can be interpreted as a band of HCP stacked material produced at the interface. The sequence of 


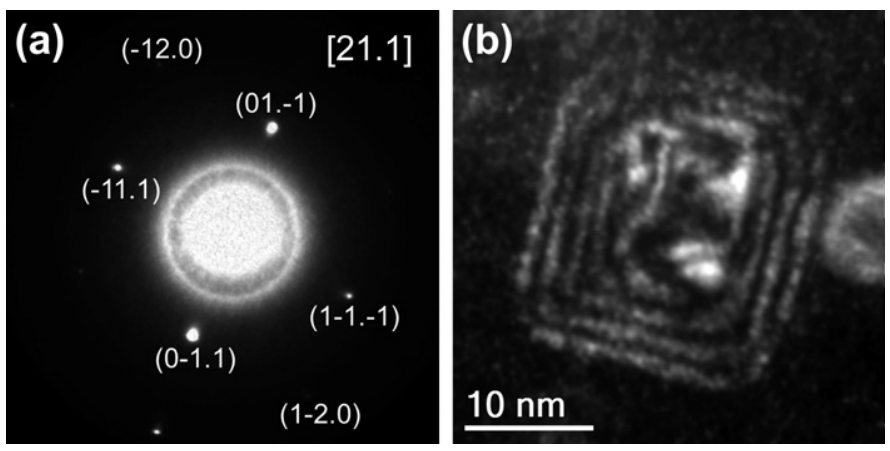

Figure 4.27 (a) Nanodiffraction pattern of an individual Au/Pd nanoparticle. (b) Highresolution weak-beam Dark-Field images of the nanoparticles. (Reprinted with permission from Deepak et al. (2011a). Copyright (2011) Elsevier).

the thickness fringes is not homogeneous, which is due to the presence of lattice defects (Mayoral et al., 2010).

Figures 4.28a and 4.28b show a three-layered gold-palladium particle in its $<110>$ orientation in the HAADF and BF-STEM modes, respectively. Profiles of the first and second layers of the particle are shown in Figures $4.28 \mathrm{c}$ and $4.28 \mathrm{~d}$, respectively. The profiles show a very rough surface; this can only be explained if we consider two factors. First, atoms of gold migrated to the surface, causing an increase in signal in those particular columns. Second, there are incomplete columns of atoms (e.g., steps) on the surface.

The BF-STEM images of the three-layer nanoparticle $\left(\mathrm{Au}_{1} \mathrm{Pd}_{3}\right)$ are shown in Figures 4.29a and 4.29b; the corresponding HAADF images are shown in Figure 4.29c. The incident electron beam direction is along [110]. The side surfaces of the particles can be indexed as $\{111\}$ and $\{100\}$ planes (labeled in Figures 4.29a and 4.29d). The center of the nanoparticles is round, but the exterior surface is square-shaped. The real shape of these particles can be associated with a truncated octahedron. This is extremely interesting since the individual metals themselves will tend to form icosahedral or decahedral shapes. Thus, the structure can be changed with the chemical composition. The nanoparticles have defects at the $\{111\}$ surface in all directions (Figure 4.29a). A higher magnification of the BF-STEM image in Figure $4.29 \mathrm{~b}$ shows the defects clearly, and along with the HAADF image shown in Figure 4.29c, gives a clear indication of the variation in the stacking sequence that leads to the stacking faults. Although the standard stacking sequence is $\mathrm{ABCABC}$ in FCC structures, in the regions marked in the images (see Figures 4.29b and c), this sequence is not followed. Instead, the sequence becomes ABCAB... (FCC-HCP structure, as seen in 

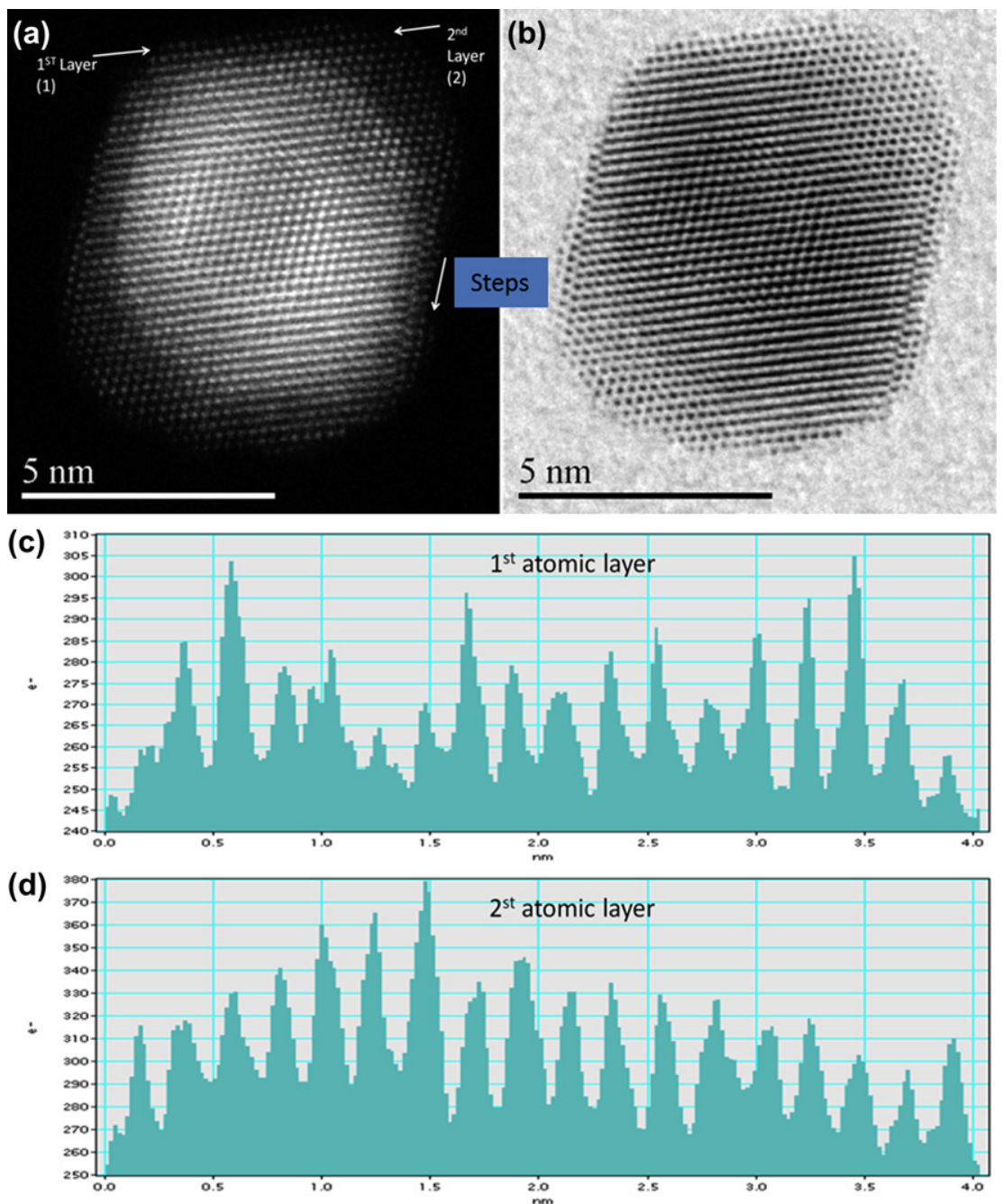

Figure 4.28 (a) HAADF- and (b) BF-STEM images of a $\mathrm{Au}_{1} \mathrm{Pd}_{4}$ nanoparticle; the images in (c) and (d) show the intensity of the first two atomic layers of the nanoparticle. (Reprinted with permission from Deepak et al., (2011a). Copyright (2011) Elsevier). (See the color plate).

Figure 4.29b) and ABCAB... (Figure 4.29c). This corresponds to stacking faults. In addition, the presence of Shockley partial dislocations (SPDs) can be observed (see Figure 4.29a). The formation of stacking faults in goldpallidium core-shell structures has been shown to be linked to SPDs (Ding et al., 2010). A partial dislocation is accompanied by the presence of a stacking fault. It may be noted here that the large strain involved in the 

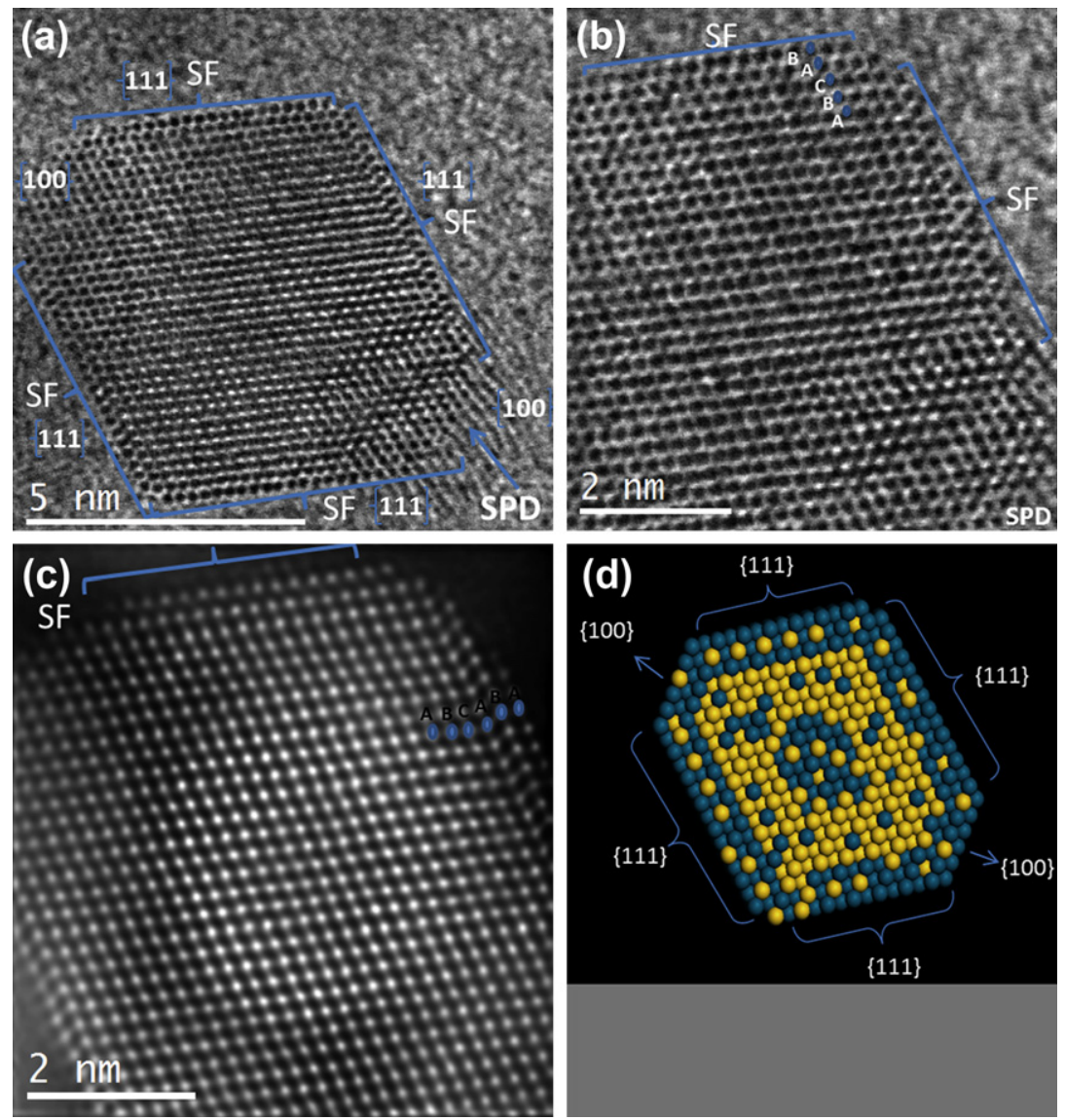

Figure 4.29 The BF-STEM images of the three-layer nanoparticle (a) and (b) and (c) the HAADF image. A higher magnification of the BF-STEM image of (a) is shown in (b) and the HAADF image shown in (c) clearly indicates the variation in the stacking sequence leading to the stacking faults, which is in combination with Shockley partial dislocations. (d) Atomistic model of $\mathrm{Au} / \mathrm{Pd}$ modified cuboctahedron with the three layers and showing the $\{100\}$ and $\{111\}$ facets: a palladium-rich inner core, a gold-rich intermediate shell, and a palladium-rich exterior shell. (Reprinted with permission from Deepak et al. (2011a). Copyright (2011) Elsevier). (See the color plate).

formation of the three-layer structures may be alleviated to some extent by the formation of such stacking faults and related defects. The stacking faults are also evident for the $\mathrm{Au}_{1} \mathrm{Pd}_{4}$ trilayer particles.

Figures $4.30 \mathrm{a}$ and $4.30 \mathrm{~b}$ are the HAADF-STEM and the BF-STEM images, which show a variation in the stacking sequence from the previously mentioned case. Here the stacking sequence of $\mathrm{ABCBA}$ is produced by twinning and layer $\mathrm{C}$ corresponds to the twinning mirror plane. 

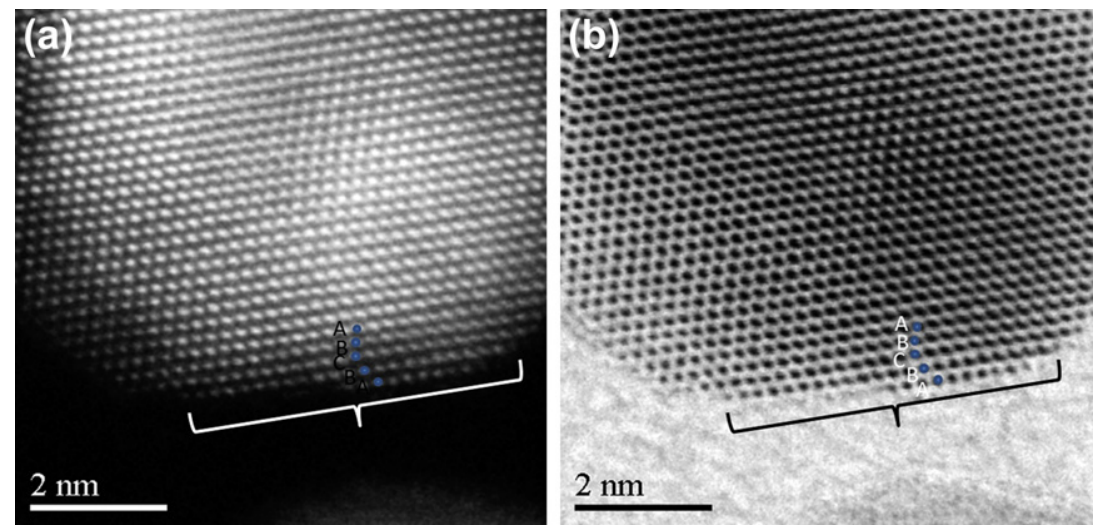

Figure 4.30 HAADF and BF-STEM images of $\mathrm{Au}_{1} \mathrm{Pd}_{4}$ nanoparticle showing the presence of twinning. (Reprinted with permission from Deepak et al. (2011a). Copyright (2011) Elsevier). (For color version of this figure, the reader is referred to the online version of this book).

It is indeed interesting to note that the ultimate resolution of observing individual atoms/small clusters has been achieved using probe aberrationcorrected STEM. Figure 4.31 shows the very small bimetallic clusters of palladium-iridium (inside the circle). The size of the cluster is just about $\sim 2 \mathrm{~nm}$. It is interesting to note that based on the Z-contrast, the two different atoms can be distinguished very clearly (palladium, $Z=46$; iridium $Z=77$ ). In addition, it is also possible to locate individual atoms of iridium

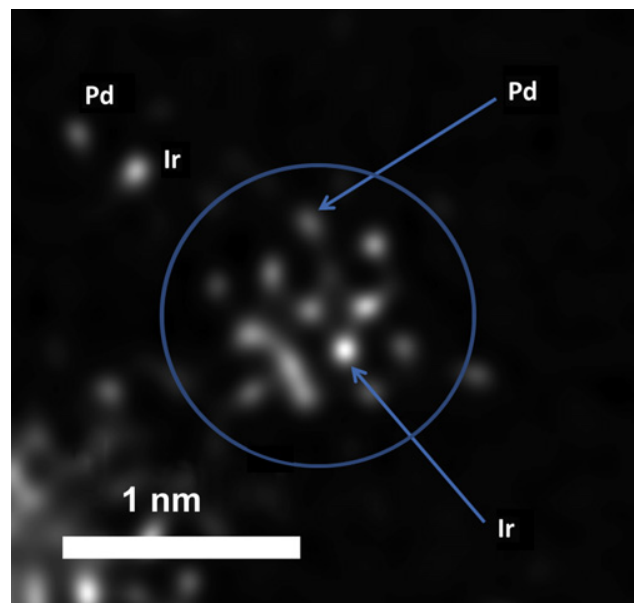

Figure 4.31 Clusters palladium-iridium are shown (inside the circle). It is possible to distinguish each element based on the Z-contrast (palladium $=46$, iridium $=77$ ). Notice also the presence of individual atoms (outside and close to the circle). (For color version of this figure, the reader is referred to the online version of this book). 
and palladium in the vicinity of these small clusters (outside the circle) (Mayoral et al., 2011).

\subsection{In Situ Heating Experiments of Bimetallic Nanoparticles and Clusters}

One of the most exciting experiments performed in an electron microscope uses the in situ heating method. This method provides a great deal of information on the behavior of catalysts and other nanomaterials. With the advent of aberration-corrected electron microscopes that provide subAngstrom image resolution, it is of great interest to study the behavior of materials at high temperatures while maintaining the resolution capabilities of the microscope. The in situ heating experiments help overcome several performance problems usually associated with standard heating stage technologies.

A typical example of the heating stage experiments is shown for the three-layered palladium-gold-palladium nanoparticles. Figure 4.32 shows a series of HAADF and $\mathrm{BF}$ images recorded at magnifications from $500 \mathrm{kx}$ to $8 \mathrm{Mx}$ as temperatures gradually increased from $350{ }^{\circ} \mathrm{C}$ to $900{ }^{\circ} \mathrm{C}$ in steps of $50^{\circ} \mathrm{C}$. Figures $4.32 \mathrm{a}-\mathrm{c}$ show the behavior of a cluster of gold-palladium particles during this heating sequence. The core-shell structure is clear in Figure $4.32 \mathrm{a}$ at $350^{\circ} \mathrm{C}$ but has disappeared in Figure $4.32 \mathrm{~b}$ at a temperature of $550{ }^{\circ} \mathrm{C}$ as the particles homogenize by diffusion. Particle A shows little change in size, whereas particle $\mathrm{B}$ shows a suggestion of growth; however, these effects are small and may simply result from a small rearrangement in shape at this temperature. Two small clusters of gold-pallidium atoms appear at the positions marked with arrows; these are probably a result of the migration of atomically dispersed species not seen at this magnification. Figure $4.32 \mathrm{c}$ shows the particle grouping at $700{ }^{\circ} \mathrm{C}$. Remarkably, the center particle diminishes greatly in size, with the growth of a new grain on the adjacent surface of the larger particle. The two particles did not sinter together, even though they were apparently in contact at the higher temperature. New particle 1 grew also, but new particle 2 did not change appreciably after its initial formation at the lower temperature. The coreshell structure was not apparent in any particle, suggesting full alloying occurred after time at the higher temperature. The set of BF images in Figures $4.32 \mathrm{~d}-\mathrm{f}$, corresponding to the above HAADF set, provides additional information to the characterization of the behavior. Crystal lattice can be resolved in $\mathrm{BF}$ images at orientations somewhat farther away from the precise orientation required for HAADF images. In Figure $4.32 \mathrm{~b}$ the 

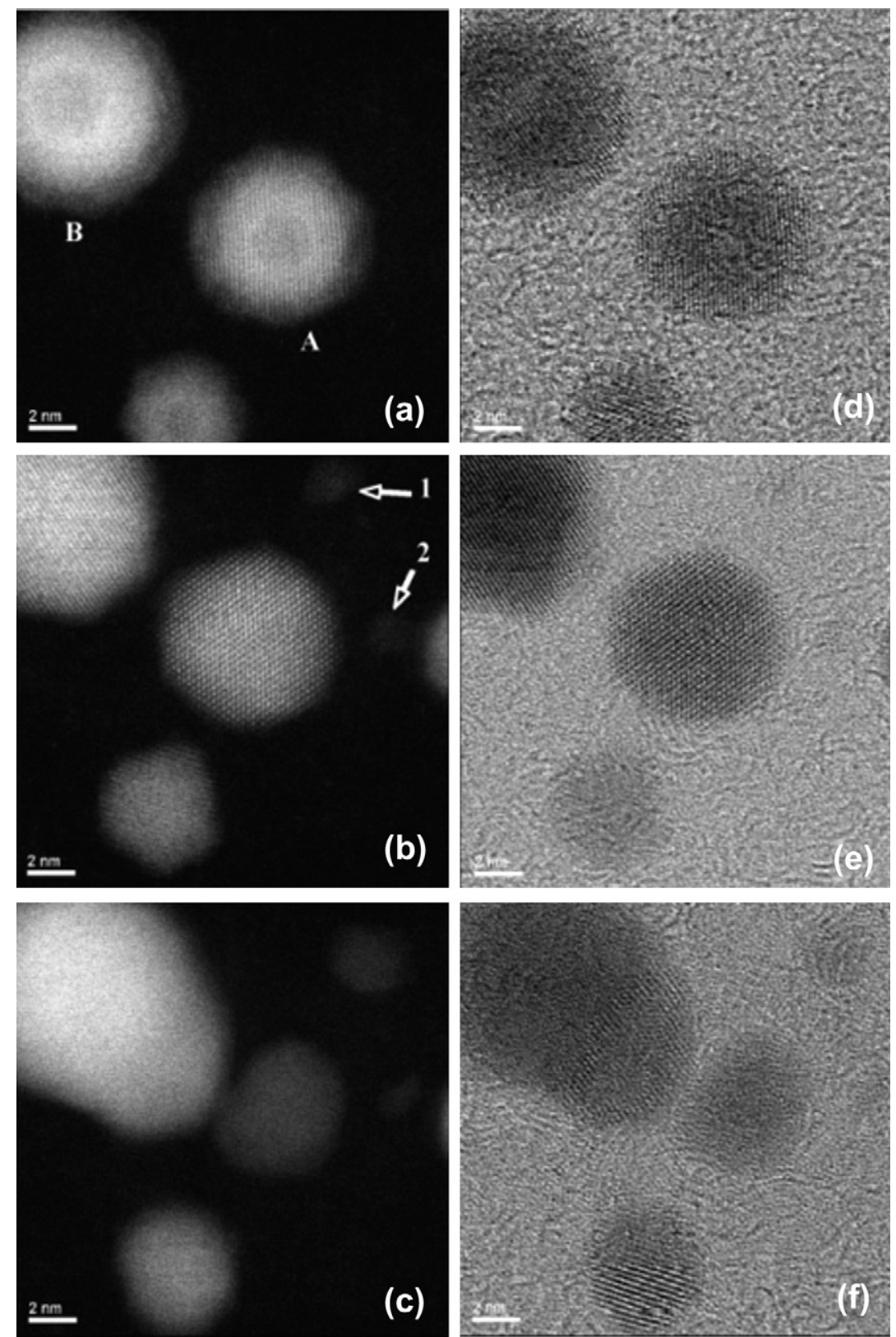

Figure 4.32 Series of HAADF and BF image pairs showing heating effects on threelayer Au-Pd particles at $350{ }^{\circ} \mathrm{C}\left[(\mathrm{a})\right.$ and (d)], $550{ }^{\circ} \mathrm{C}\left[(\mathrm{b})\right.$ and (e)], and $700{ }^{\circ} \mathrm{C}[(\mathrm{c})$ and (f)]. (Reprinted with permission from Allard et al. (2009). Copyright (2009) Wiley). 
beginning of growth of particle $\mathrm{B}$ is apparent, where the new material is not at the same crystal orientation as the original particle, in which the lattice is visible. At $700{ }^{\circ} \mathrm{C}$ (Figure 4.32c), particle B shows crystal lattice fringes and is apparently two separate grains. Changes in lattice structure visible from one image to the next are largely the result of slight shifts in orientation of the particles because of the thermal effects (Allard et al., 2009). In the presented example, the clear homogenization of the three-layer gold-pallidium particles was an expected result, explained by a diffusion mechanism. This suggests that a direct diffusive reconstruction of an alloy particle has been directly observed. The reasons for the unexpected failure of two primary particles to sinter but instead to show growth of one particle at the expense of the other have not been well understood. One possibility is that particles acquire some charge during the observations, thereby affecting the surface energy. The growth behavior is reminiscent of Ostwald ripening effects, although the system involves particle behavior on a uniform surface, whereas Ostwald ripening generally involves particle coarsening in solid or liquid solutions.

\subsection{Multimetallic Systems}

Although bimetallic systems are widely produced and studied for various applications, it may also be possible to form multimetallic nanoparticles under specific experimental conditions by careful control of the experimental parameters. A typical example is the case of multimetallic Pd$\mathrm{Au}-\mathrm{FePt}$. Figure $4.33 \mathrm{a}$ and $4.33 \mathrm{~b}$ show the TEM and HAADF-STEM images of the Pd-Au-FePt nanoparticles. The HAADF-STEM images reveal the darker palladium core and the brighter Au-FePt shells. The thickness of the individual layers themselves are core, pallidium $5 \mathrm{~nm}$; shell, gold $2 \mathrm{~nm}$; and shell FePt $2 \mathrm{~nm}$. EDS line scans reveal the various layers present in the nanoparticle (Figure $4.33 \mathrm{c}$ ). The EDS line scans also reveal that there is no interatomic diffusion between the gold and FePt layers, proving indeed that the structure is multilayered. The catalytic properties of such systems in the case of the oxygen reduction reaction have been studied and have been shown to have increased tremendously compared to the corresponding monometallic systems (Ding et al., 2012).

\subsection{Conclusions}

With the advent of aberration-corrected electron microscopy the elucidation of the structure of bimetallic and even multimetallic nanoparticles down 


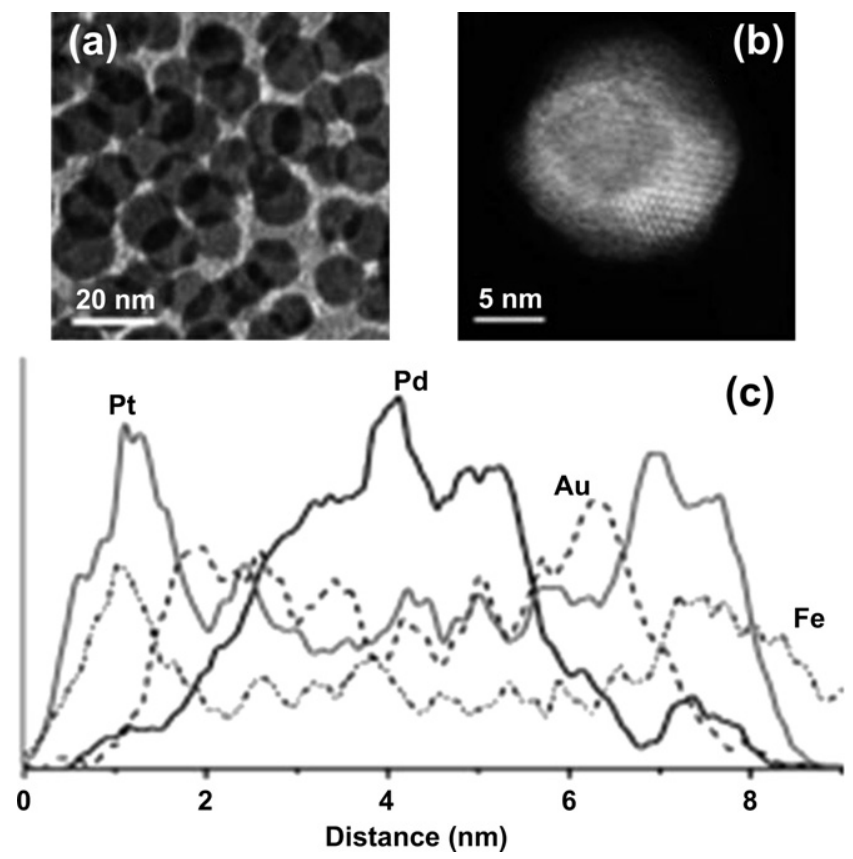

Figure 4.33 (a) TEM and (b) HAADF-STEM image of a single Pd-Au-FePt nanoparticle. (c) High-resolution line scan EDS analysis across the nanoparticle. (Reprinted with permission from Mazumder et al. (2010). Copyright (2010) Wiley).

to a single atom is now possible. Combined with spectroscopic techniques such as EDS and EELS the information obtained on the structure, stoichiometry, chemical composition, and the nature of core-shell/alloy formation of bimetallic nanoparticles is now unprecedented. More recent and exciting trends based on in situ dynamic experiments such as the heating stage studies, definitely promise an exciting future for the case of bimetallic nanoparticles and their enhanced understanding toward various applications including catalysis. It is hoped that with all the intricacies associated with the elucidation of various applications such as catalysis, a realistic progress in this area of research is now truly possible.

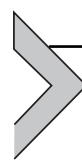

\section{ELECTRON MICROSCOPY OF LAYERED MATERIALS}

\subsection{Introduction}

Transition metal chalcogenides such as $\mathrm{MoS}_{2}$ and $\mathrm{WS}_{2}$ are quasi-2D compounds. Atoms within a layer are bound by strong covalent forces, 
whereas individual layers are held together by van der Waals interactions. Although a large number of polytypes are observed for the transition metal chalcogenides, the most notable experimentally observed polytypes for $\mathrm{MoS}_{2}$ and $\mathrm{WS}_{2}$ based on the stacking sequence of the layers are a hexagonal polymorph with two layers in the unit cell, $2 \mathrm{H}(\mathrm{P} 63 / \mathrm{mmc})$ symmetry; rhombohedral with three molecular layers in the unit cell, 3R ( $\mathrm{R} 3 \mathrm{~m}$ ) symmetry; or the less common trigonal with one layer, $1 \mathrm{~T}$. For instance, $\mathrm{MoS}_{2}$ is a widely used compound with many applications in catalysis and as solid lubricants mainly because of the weak interlayer interaction of the material (Tye and Smith, 2004; Lince, 2004; Wu et al., 2010). More recent applications are hydrogen storage, as host-guest compounds, scanning tunneling microscope tips, and solid-state secondary lithium battery cathodes (Chen et al., 2001; Zak et al., 2002; Remskar et al., 2000; Homyonfer et al., 1997). The most promising and most pursued of all these applications is their use as industrial catalysts for hydrodesulfurization (HDS) of crude oil. Elimination of sulfur from petroleum feedstocks is necessary to meet the severe restrictions on the sulfur concentrations in fuels (Frey et al., 1999; Babich and Moulijn, 2003). The HDS of polyaromatic sulfur compounds or deep HDS is especially difficult for heavy oils containing high concentrations of sulfur (2-3 wt \%). Catalysts based on molybdenum sulfide are widely used in oil refineries for the HDS, hydrodenitrogenation (HDN), and hydrodeoxygenation (HDO) reactions of petroleum-derived feedstocks (Song, 2003; Li and Delmon, 1997; Gates, 1992). Because the stringent environmental legislation sets the sulfur level at $<15 \mathrm{ppm}$, new catalysts with significantly improved catalytic performance are being developed. Sulfur compounds that are known to remain in fuels such as diesel at sulfur levels below $500 \mathrm{ppm}$ include dibenzothiophene (DBT) and alkyl-substituted DBTs such as 4,6-dimethyldibenzothiophene (4,6-DMDBT) (Whitehurst et al., 1998; Turaga et al., 2003) and ongoing efforts using different Mo- and W-based catalysts are directed toward removing sulfur. $\mathrm{MoS}_{2}$ is also combined with other metals, such as cobalt, nickel, and iron, for use in HDS (Byskov et al., 1999).

\section{2. $\mathrm{MoS}_{2}$ Nanowire Catalysts}

One of the challenges in working with catalysts is obtaining direct atomicscale insights to understand their structure better, thereby enabling direct coordination with their activity. For $\mathrm{MoS}_{2}$-based catalysts this has proved elusive so far since the location of the active sites and their chemical affinity to HDS reactions is poorly understood without atomic-resolution imaging 
techniques. A solution to this provided is provided with the use of aberrationcorrected $\left(C_{s}\right)$ STEM-HAADF imaging. HAADF-STEM imaging was performed previously for model $\mathrm{MoS}_{2}$-based catalysts by Brorson et al. (2007). However, the resolution obtained in terms of imaging these catalysts was not sufficient enough (Brorson et al., 2007). Hence probe aberrationcorrected STEM imaging has been performed to image $\mathrm{MoS}_{2}$ nanowirebased catalysts to better understand the atomic structure of these $\mathrm{MoS}_{2}$-based catalysts. This technique allows the identification of direct atomic locations and hence enhances the understanding of the various catalysts, especially identifying the active sites at which catalysis occurs.

Figure 4.34a shows the HAADF-STEM image of the $\mathrm{MoS}_{2}$ nanowire catalysts supported on alumina. The $\mathrm{MoS}_{2}$ nanowire catalysts vary in stacking and range between one and three layers. Also noticeable is that the nanowires appear to be twirled or twisted. The figure shows the filtered image of the $\mathrm{MoS}_{2}$ catalyst, where the bright atom contrast arises from the Mo atoms in the individual layers of the $\mathrm{MoS}_{2}$ nanowires. The presence of the sulfur atoms, which show less contrast compared with the molybdenum atoms can be also inferred from the image. Thus the sulfur-molybdenumsulfur chevron can be identified along the entire length of the nanowire. Measuring the distance between the molybdenum-molybdenum atoms (from the bright atom contrast) shows that they correspond to a distance of $2.7 \AA$ (Figure $4.35 \mathrm{~b}$ shows the line profile along the length of the entire individual $\mathrm{MoS}_{2}$ nanowire).

Figure 4.35 shows the $\mathrm{MoS}_{2}$ nanowires with a helical structure obtained by the twirling or twisting of the individual as well as the double or triple $\mathrm{MoS}_{2}$ layers. Figures 4.35a and 4.35b show the HAADF-STEM image and the BF-STEM images of these helical $\mathrm{MoS}_{2}$ catalysts. Figure $4.36 \mathrm{c}$ shows the filtered HAADF-STEM image of the $\mathrm{MoS}_{2}$ catalyst. Interestingly, the catalytically inert basal planes of the nanowires are folded onto themselves to expose the highly active catalytic edge sites (surface atoms seen along the entire length of the nanowire as viewed along the $c$-axis). Figure $4.35 \mathrm{~d}$ is the line profile corresponding to the interatomic distances of the molybdenummolybdenum atoms (bright atom contrast), with distances of $2.7 \AA$ A between the atoms. The white arrow shows the sulfur atoms that make up the sulfurmolybdenum-sulfur chevron (Deepak et al., 2011b).

Combinated with experimental insights, theoretical simulations have contributed significantly to the fundamental understanding of $\mathrm{MoS}_{2}$-based catalysts. In the past decade, several reports have focused on the detailed edge structure, location of active sites, slab-size effects (width and thickness), 

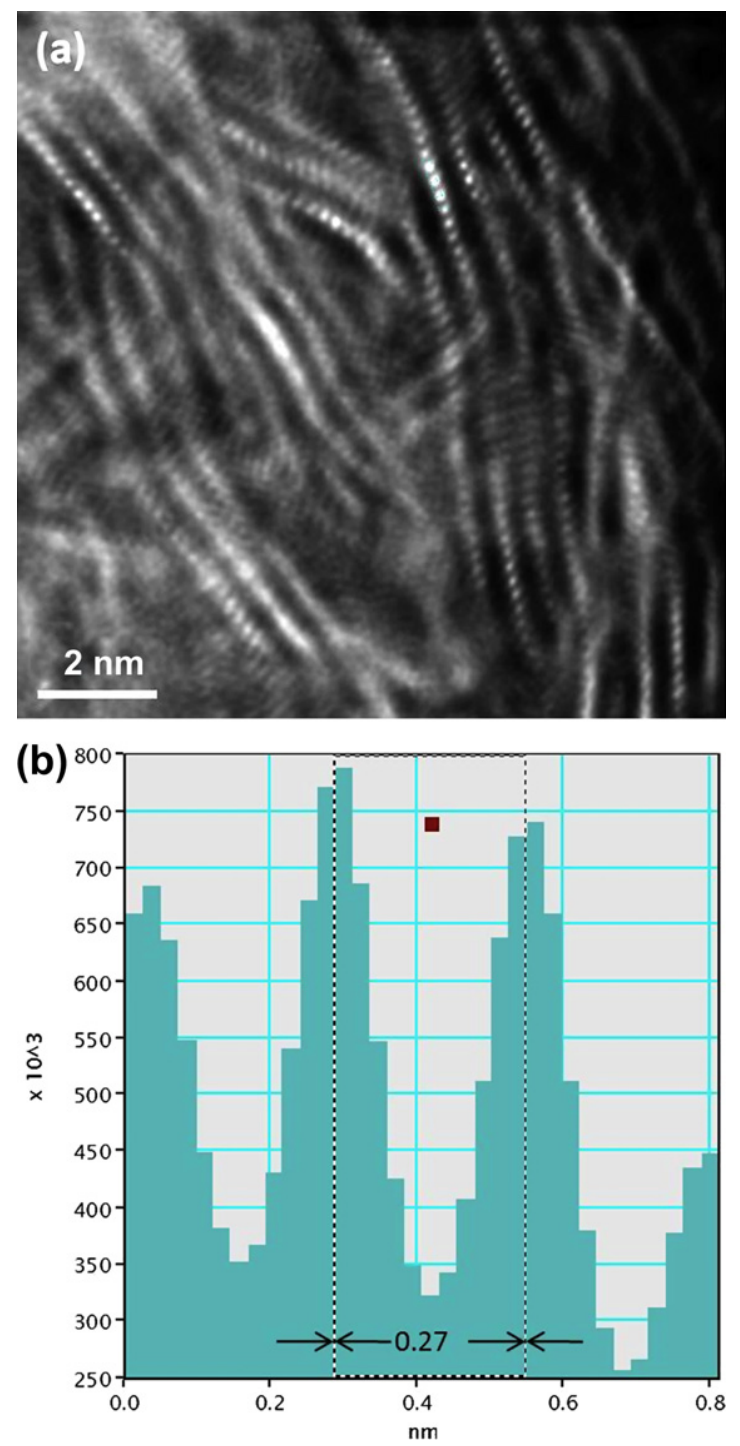

Figure 4.34 $\mathrm{MoS}_{2}$ nanowire catalysts (on $\mathrm{Al}_{2} \mathrm{O}_{3}$ support). (a) Filtered HAADF-STEM image of the $\mathrm{MoS}_{2}$ catalyst. (b) The line profile corresponding to the interatomic distances of the Mo-Mo atoms (bright atom contrast); the distances between the atoms are $2.7 \AA$. The presence of the sulfur atoms with a lesser contrast can also be seen from the image. (Reprinted with permission from Deepak et al. (2011b). Copyright (2011) Springer). (See the color plate). 

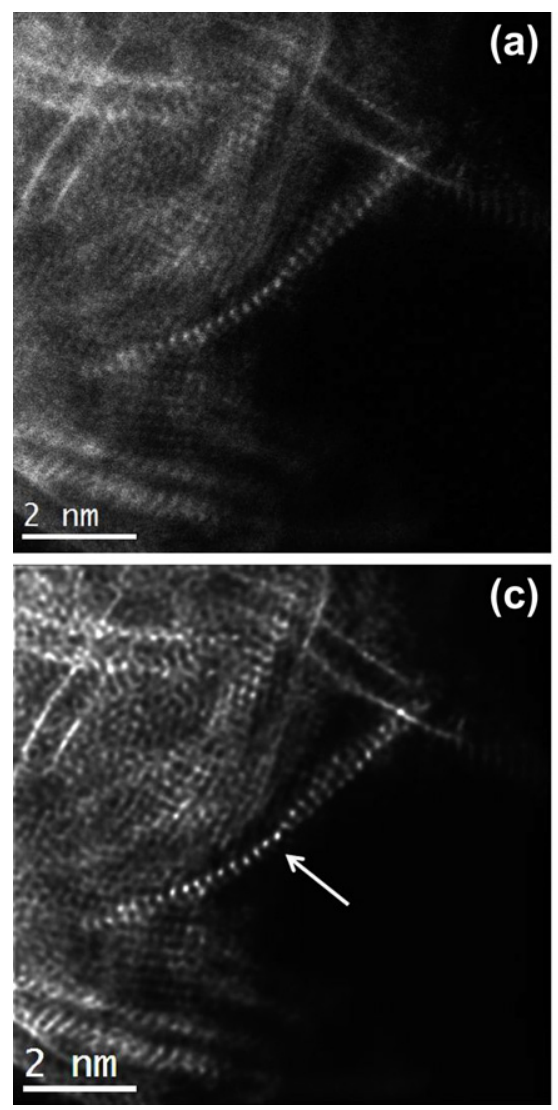

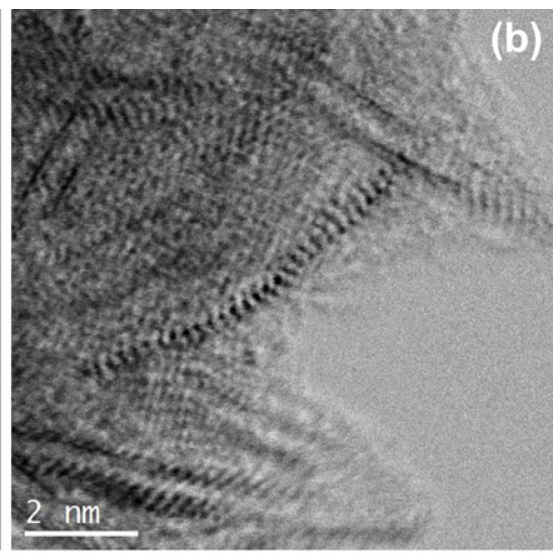

(d)

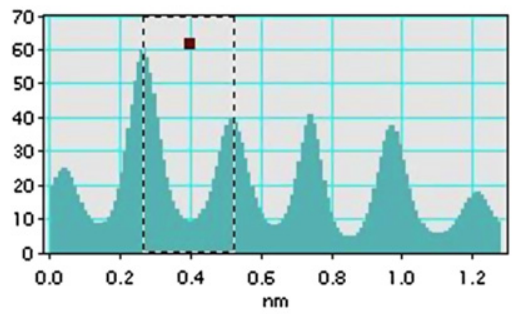

Figure 4.35 $\mathrm{MoS}_{2}$ nanowire catalysts supported on $\mathrm{Al}_{2} \mathrm{O}_{3}$. (a) HAADF-STEM image. (b) BF-STEM image of the $\mathrm{MoS}_{2}$ catalysts. (c) Filtered HAADF-STEM image of the $\mathrm{MoS}_{2}$ catalyst. (d) The line profile corresponding to the interatomic distances of the Mo-Mo atoms (bright atom contrast), the distances between the atoms are $2.7 \AA$. The white arrow shows the sulfur atoms that make up the S-Mo-S chevron. (Reprinted with permission from Deepak et al. (2011b). Copyright (2011) Springer). (See the color plate).

the effect of the hydrogen passivation, the magnetic order edge states, and the vacancies formation (Shidpour and Manteghian, 2010; Li et al., 2008; Ionescu et al., 2003; Iglic et al., 2005). In a majority of these studies, the $\mathrm{MoS}_{2}$ catalyst has been modeled through $\mathrm{MoS}_{2}$ sheets where the molybdenum appears in the forms of ions sandwiched by sulfur atoms in a trigonal prismatic coordination. Disregarding the inactive basal plane (0001), the $(10 \overline{1} 0)$ molybdenum edge (zigzag-terminated morphology) and $(2 \overline{1} \overline{1} 0)$ Mo-S edge (armchair-terminated morphology) are of particular importance because they contain the "active sites" through the existence of sulfur 
(a)

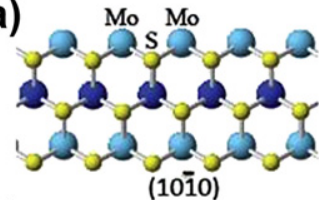

(c) (10īo)

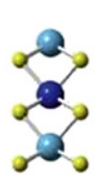

(b)

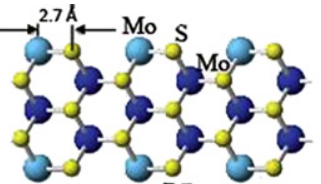

(2īio)

S

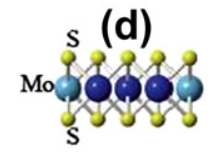

(e)

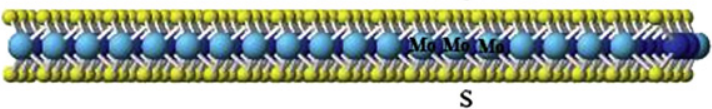

$\mathrm{S}$

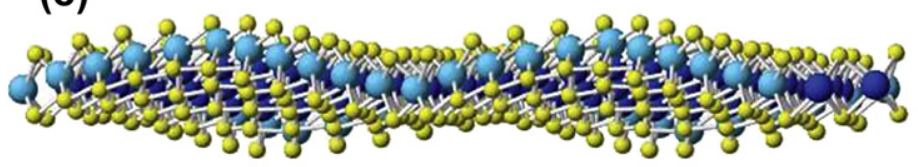

(f)

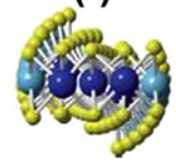

(g)

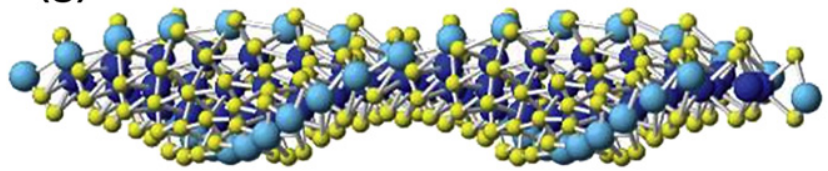

(h)

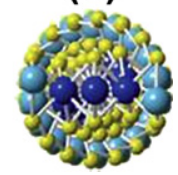

Figure 4.36 (a) and (b) show the side and transversal views of a single-sheet zigzagand armchair-terminated $\mathrm{MoS}_{2}$ nanowire. (c) Perspective top and transversal (d) views of an armchair-terminated $\mathrm{MoS}_{2}$ nanowire similar to (b). (e) and (g) Perspective side views, and ( $f$ ) and ( $h$ ) transversal views of a rippled (helical) armchair nanowire. The Mo-edge atoms appear light-colored in the figure compared with the other Mo-atoms (dark-colored). (Reprinted with permission from Deepak et al. (2011b). Copyright (2011) Springer). (See the color plate).

vacancies. The vacancies on the edges and the intrinsic magnetic order certainly play a key role on the catalytic properties of $\mathrm{MoS}_{2}$ clusters and nanoribbons. Armchair nanoribbons could be metallic and exhibit a magnetic moment. However, when passivated with hydrogen, they become semiconducting. Zigzag nanoribbons, on the other hand, are metallic and exhibit unusual magnetic properties regardless of their passivation (Botello-Mendez et al., 2009; Shidpour and Manteghian, 2010; Li et al., 2008; Ionescu et al., 2003). It has been suggested that the presence of the vacancies plays a much more substantial role on the magnetization creation, while the magnetic properties can be drastically affected by the size of the nanostructures themselves. In this connection, it must also be noted that electronic properties and edge states in stacked $\mathrm{MoS}_{2}$ multilayer nanowires and found them to exhibit metallic behavior (Iglic et al., 2005). State-of-the-art simulations indicate that the existence of the metallic edge 
states seems to be crucial in order to raise the catalytic activity of $\mathrm{MoS}_{2}$ nanostructures. Iglic et al. (2005) have proposed the possibility of $\mathrm{MoS}_{2}$ structures with helical and twisted morphologies but did not study their electronic or magnetic properties or consider them of importance because of its unproven existence in nature so far (Kralj-Iglic et al., 2004). Thus, the presence of rippled and twisted or helical morphologies of $\mathrm{MoS}_{2}$ has not been observed previously (Iglic et al., 2005; Kralj-Iglic et al., 2004; Kisielowski et al., 2011), and advanced electron microscopic techniques have been used until recently to image such catalyst systems with unprecedented structural details (Hansen et al., 2011; Galvan et al., 2011).

Based on the HAADF-STEM images, various atomic models were built to illustrate the different morphologies found in the experimentally observed $\mathrm{MoS}_{2}$ catalysts discussed previously. Figures $4.36 \mathrm{a}$ and $4.36 \mathrm{~b}$ show the side and transversal views of zizzag- and armchair-terminated $\mathrm{MoS}_{2}$ sheets, respectively. It has been shown that the [10 10$]$ edge plane is more stable than the $[2 \overline{1} \overline{1} 0]$ edge plane. However, according to the experimental images, the molybdenum atoms are aligned along the [2 $\overline{1} \overline{1} 0]$ direction, a characteristic that it is possible only for an armchair-terminated slab. For this reason, the first model corresponds to a single-layer armchair-terminated $\mathrm{MoS}_{2}$ nanowire. Figures 4.36 a-d show different views of an armchair nanowire consisting of a five-atomic layer $\mathrm{S}-\mathrm{Mo}-\mathrm{S}$ sandwich. The relative distance between the molybdenum edge atoms in our model is $2.7 \AA$, very similar to the experimental value. The experimentally observed nanowires are found mostly in bundles with thicknesses ranging from two to eight single-sheet $\mathrm{MoS}_{2}$ layers, a varying width ranging from two to six atomic layers, and a length ranging from 20 to $50 \mathrm{~nm}$. The slightly distorted quasi-planar nanowire structures appear to be stabilized by the neighboring layers and are configured almost as in the bulk, with a distance between the singlesheet layers ranging from 5.3 to $6.2 \AA$. In some bundles the bending/tilting of some nanowires is observed, a fact that leads to a second model. Figures 4.36e and 36f show a perspective and transversal view of a rippled nanowire. These nanostructures are indeed considerably elongated and can also be found in bundles or isolated (Figure 4.36a). In contrast with the previous model, the distances between the edges of two neighboring nanowires is affected by the tilting and therefore are not uniform. Interestingly, the distance between the axes of two neighboring rippled nanowires is nearly the same as for the flat nanowires. When the bending of the nanowires is more pronounced, we observed the irregular twisting of the $\mathrm{MoS}_{2}$ nanostructures. Figures $4.36 \mathrm{~g}$ and $4.36 \mathrm{~h}$ show a perspective and 
transversal view of the third experimentally observed morphology that corresponds to a helical or twisted nanowire. Indeed, some of the distorted nanowires are a mixture between the rippled and the helical ones. DFT calculations of the electronic structure of the $\mathrm{MoS}_{2}$ spirals suggest that the twisting produces a transition from semiconductor to semimetal behavior. This effect could be attributed to a distortion produced by the rotation applied to the nanowire; due to this the $\mathrm{MoS}_{2}$ spirals should have improved catalytic activity. The existence of this new atomic structure changes completely the landscape of the location of the catalytically active sites, opening up the possibility for new $\mathrm{MoS}_{2}$-based nanocatalysts (Galvan et al., 2011).

\subsection{Co-Doped $\mathrm{MoS}_{2} / \mathrm{WS}_{2}$ Nanowire Catalysts}

While unpromoted $\mathrm{MoS}_{2}\left(\mathrm{WS}_{2}\right)$ catalysts do not possess high catalytic activity, a very significant increase in their activity can be achieved by adding cobalt or nickel. The use of metal nanoparticles in HDS reactions has been studied by incorporating a variety of transition metals, such as molybdenum, nickel, $\mathrm{Ni} / \mathrm{Fe}, \mathrm{Ni} / \mathrm{W}, \mathrm{Mo} / \mathrm{W}$, and $\mathrm{Mo} / \mathrm{Co}$, which are able to hydrodesulfurize thiophene and DBT in good yields (Yoosuk et al., 2008; Senevirathne et al., 2007; Escalona et al., 2006; Olivas et al., 2006; Dhas and Suslick, 2005; Farraghher, 1979). Of particular interest is the case of Co-promoted $\mathrm{MoS}_{2} / \mathrm{WS}_{2}$-supported catalysts. Several investigations have been directed toward understanding the nature and function of the Copromoter atoms in $\mathrm{MoS}_{2} / \mathrm{WS}_{2}$-based catalysts. The difficulty for many years was to understand in detail the catalytic properties of the different types of cobalt-molybdenum-sulfur structures since direct atom-resolved insight was not available previously. Techniques such as extended X-ray absorption fine structure and X-ray photoelectron spectroscopy have been used to obtain information on the promoter atoms (example: $\mathrm{Ni} / \mathrm{Co}$ ) incorporated within the catalysts previously (example: $\mathrm{MoS}_{2} / \mathrm{WS}_{2}$ on $\mathrm{Al}_{2} \mathrm{O}_{3}$ ). Only recently has such insight provided by scanning tunneling microscopy along with density functional theory; this has contributed greatly to improve fundamental knowledge of the $\mathrm{MoS}_{2}$ and cobaltmolybdenum-sulfur catalyst structures (Topsøe, 2007). A direct solution to this is now provided using aberration-corrected HAADF-STEM imaging, which can be used to image individual cobalt atoms present in the lattice of $\mathrm{MoS}_{2} / \mathrm{WS}_{2}$ nanowire catalysts directly. The identification is also supported by performing atomic-resolution energy dispersive X-ray analysis (STEM-EDAX), which can provide reliable and precise chemical 

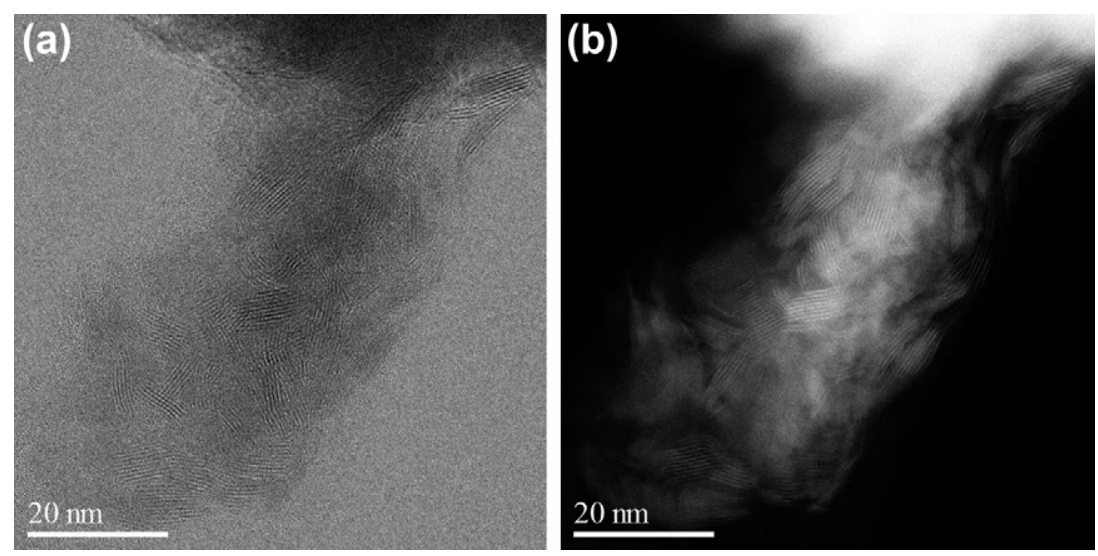

Figure 4.37 (a) and (b) show the low-magnification BF-STEM and HAADF-STEM images of the Co-doped $\mathrm{MoS}_{2}$ nanowire catalysts. (Reprinted with permission from Deepak et al. (2011b). Copyright (2011) Springer).

information to distinguish the presence of the individual promoter/dopant atoms in the lattice of the host (Deepak et al., 2011c). Figures 4.37a and 4.37b show the low-magnification BF-STEM and HAADF-STEM images of the Co-doped $\mathrm{MoS}_{2}$ nanowire catalysts.

A closer look at the nanowires is provided by the high-resolution STEM images in Figure 4.38. Figures 4.38a and 4.38b show the BF-STEM and HAADF-STEM images of the Co-doped $\mathrm{MoS}_{2}$ nanowires. Interesting variations in the atomic spacing are revealed from the image (Figure 4.39b) and the line profile (Figure 4.38c) drawn along the row of the bright atomic columns. The spacing between the atoms varies from $2.34 \AA( \pm 0.1 \AA)$ to $2.7 \AA$ ( $\pm 0.1 \AA$ ) (atoms A and B vs. B and C) (bright atom contrast revealing the molybdenum-cobalt-molybdenum interatomic distances). The final molybdenum-molybdenum interatomic distance is $2.70 \AA( \pm 0.1 \AA)$ (atoms $\mathrm{C}$ and $\mathrm{D})$. This is indeed interesting and can be explained in terms of the substitution of cobalt into the lattice of molybdenum, occupying the molybdenum sites. In addition, the difference in the atom contrast (between molybdenum and cobalt, Figure 4.39b), which decreases upon going from the $\mathrm{Mo} \rightarrow \mathrm{Co}$ and subsequently increases from $\mathrm{Co} \rightarrow \mathrm{Mo}$, is also notable. The interlayer distance as measured from the line profile of the individual layers is $5.84 \AA$. The variations on the HAADF contrast cannot be explained on the basis of thickness changes. Therefore, the most important contributions should originate from compositional variations, revealed by the difference in atomic number $(Z)$ that exists among molybdenum (atomic number $=42$ ) and cobalt (atomic number $=27$ ) (HAADF intensity is 

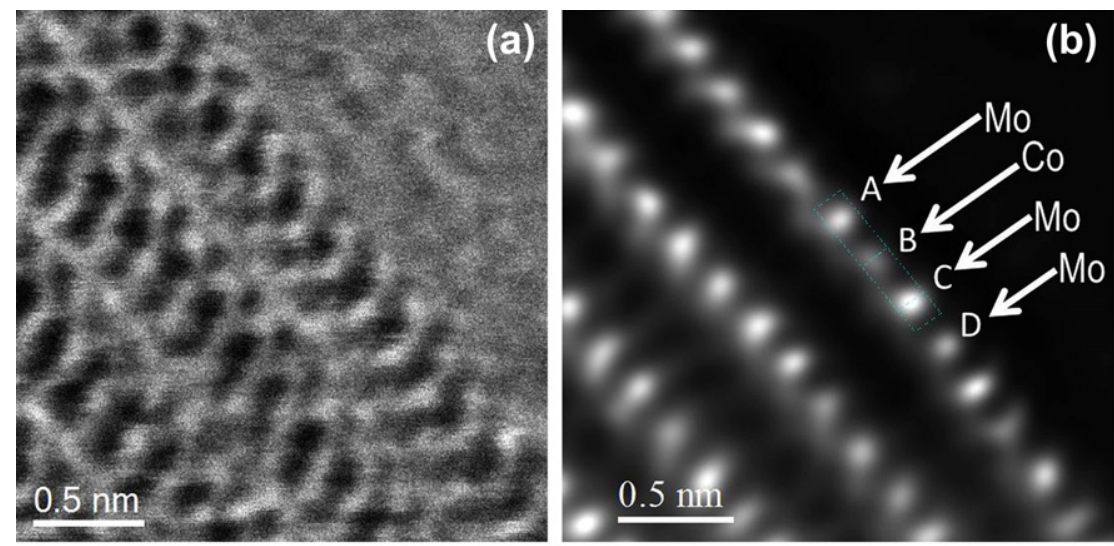

(c)

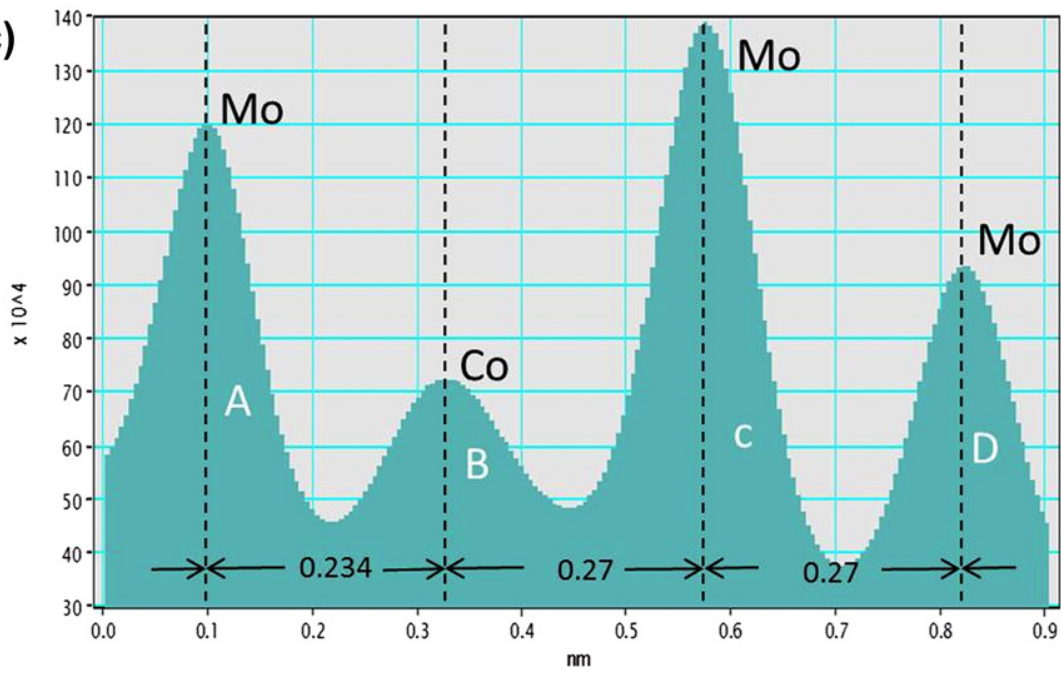

Figure 4.38 (a) and (b) BF-STEM and HAADF-STEM images of the Co-doped $\mathrm{MoS}_{2}$ nanowire catalysts (unsupported catalyst). (c) Corresponding line profiles. The spacing between the $\mathrm{Mo} \rightarrow \mathrm{Co} \rightarrow \mathrm{Mo} \rightarrow \mathrm{Mo}$ atoms as revealed by the line profile is $2.34 \AA \pm 0.1 \AA$ (Mo-Co); $2.7 \AA \pm 0.1 \AA$ (Co-Mo); and $2.7 \AA \pm 0.1 \AA$ (Mo-Mo) (atoms A-B-C-D). The cobalt atoms have a lesser intensity compared the molybdenum atoms as a consequence of the difference in their respective atomic numbers. (Reprinted with permission from Deepak et al. (2011b). Copyright (2011) Springer). (See the color plate).

considered to be proportional to $Z^{1.7}$ ). Finally, it should be noted that in between the layers, the presence of the sulfur atoms that make up the sulfurmolybdenum-sulfur structure can be inferred from their lighter contrast compared with the brighter Mo and Co atoms. Figures 39a and 39b shows the low-magnification BF-STEM and HAADF-STEM images of the 

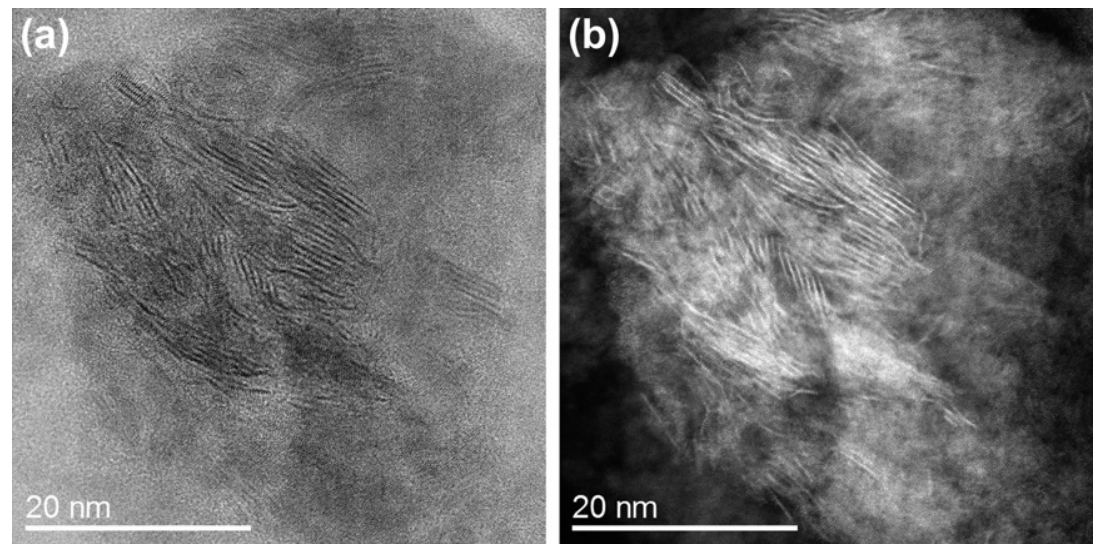

Figure 4.39 (a) and (b) Low-magnification BF-STEM and HAADF-STEM images of the Co-doped $\mathrm{WS}_{2}$ nanowire catalysts supported on alumina. (Reprinted with permission from Deepak et al. (2011b). Copyright (2011) Springer).

Co-doped $\mathrm{WS}_{2}$ nanowire catalysts supported on alumina. Large amounts of the nanowires can be clearly seen in the images.

Figure 4.40 shows the high-resolution BF-STEM and HAADF-STEM images of the Co-doped $\mathrm{WS}_{2}$ nanowire catalyst. Variations in the interatomic spacing are observed from the image (Figure 4.40b) and the line profile (Figure 4.40c) drawn along the row of the bright atomic columns. The spacing between the atoms varies as follows: $2.24 \AA( \pm 0.1 \AA$ for spacing A-B), $-2.72 \AA( \pm 0.1 \AA)$ for spacing B-C, $-2.72 \AA( \pm 0.1 \AA)$ for spacing C-D, and $-2.24 \AA$ ( $( \pm 0.1 \AA)$ for spacing D-E.

This variation in the interatomic distances can be explained in terms of the substitution of cobalt in the lattice of tungsten (akin to the case of cobalt atoms substituting molybdenum as explained above). In addition, the difference in the atom contrast (between tungsten and cobalt (Figure 4.41b) atoms $\mathrm{W}-\mathrm{Co}-\mathrm{W}-\mathrm{Co}-\mathrm{W}$ [A-B-C-D-E]) can be noted. The variations are due to the difference in atomic number $(Z)$ between tungsten (atomic number $=74$ ) and cobalt (atomic number $=27$ ). Also, the intensity variations in the HAADF line scans is dependent on the thickness of the atomic layers themselves (whether it is one, two, or three layers). This is also the reason that the $\mathrm{Mo} / \mathrm{W}$ bright atom contrast appears elongated (two or more layers are twirled and hence produce this type of effects; see for example Figure 4.40b [for the Co: $\mathrm{WS}_{2}$ case] and Figure 4.40b [for the Co: $\mathrm{MoS}_{2}$ case], where this entanglement of the $\mathrm{WS}_{2} / \mathrm{MoS}_{2}$ layers can be clearly seen). 

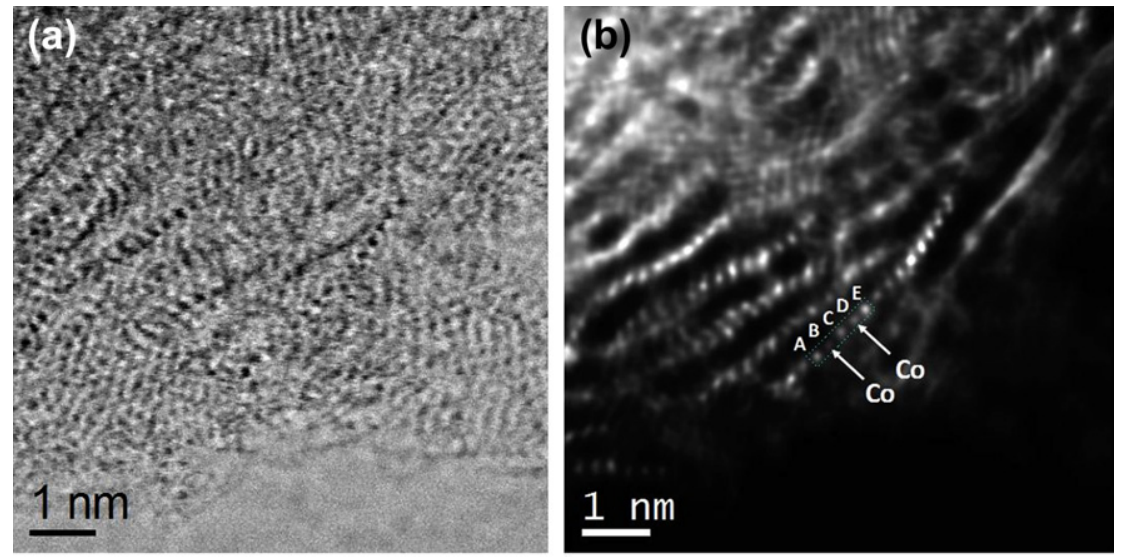

(c)

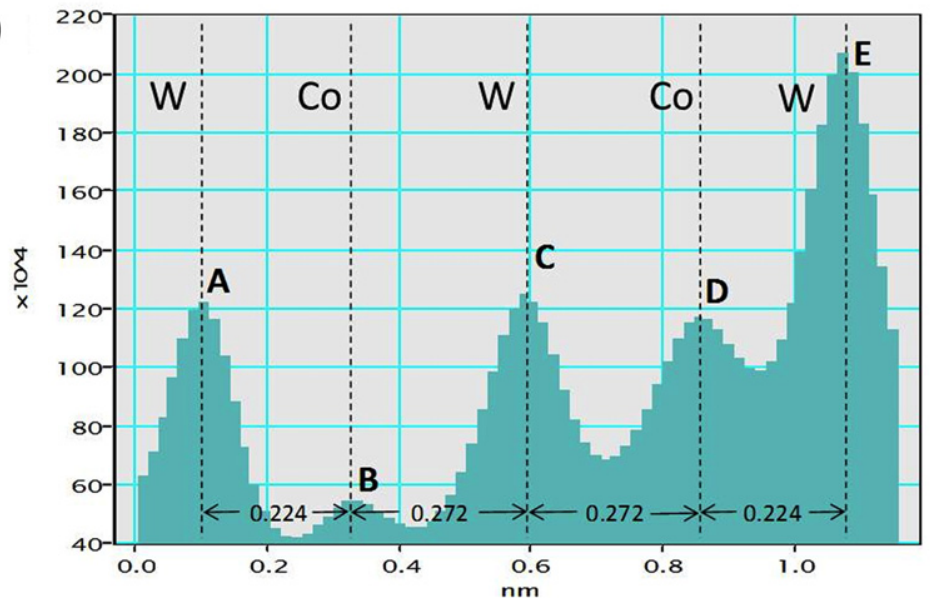

Figure 4.40 (a) and (b), BF-STEM and HAADF-STEM images of the Co-doped $\mathrm{WS}_{2}$ nanowire catalysts (supported on $\mathrm{Al}_{2} \mathrm{O}_{3}$ ) and (c) corresponding line profiles. The spacing between the W-Co-W-Co-W atoms as revealed by the line profile is $2.24 \pm 0.1 \AA$ $(\mathrm{W}-\mathrm{Co}), \quad 2.72 \pm 0.1 \AA$ (Co-W), $2.72 \pm 0.1 \AA$ (W-Co), $2.24 \pm 0.1 \AA$ (Co-W), (atoms $A \rightarrow B \rightarrow C \rightarrow D \rightarrow E$ ). The Co atoms have a lesser intensity in comparison with the $W$ atoms because of the difference in their respective atomic numbers. (Reprinted with permission from Deepak et al. (2011b). Copyright (2011) Springer). (See the color plate).

Figure 4.41b shows the EDAX line scan for one of the individual Codoped $\mathrm{WS}_{2}$ nanowires (the image is shown in Figure 4.41a). The characteristic $\mathrm{W}(\mathrm{M})$ and the Co $(\mathrm{K})$ signals are obtained from the line scans indicating the presence of tungsten and cobalt in the individual $\mathrm{WS}_{2}$ nanowire (cobalt incorporated at the tungsten sites). The EDAX spectra thus support the previous HAADF-STEM observations that cobalt is indeed present in the lattice of $\mathrm{WS}_{2}$. The identification of cobalt present uniformly 

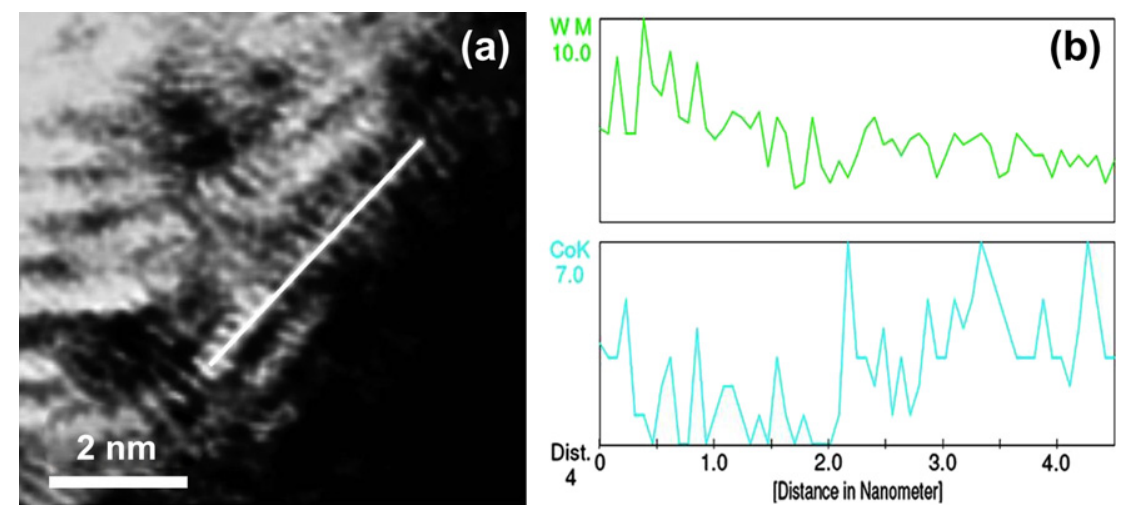

Figure 4.41 EDAX line scans carried out on an individual Co-doped $\mathrm{WS}_{2}$ nanowire catalyst. The presence of the characteristic and distinct signals of $W(M)$ and Co (K) have been identified from the line scan (Co present at the W-sites). (Reprinted with permission from Deepak et al. (2011b). Copyright (2011) Springer). (See the color plate).

throughout the $\mathrm{WS}_{2}$ catalysts was ascertained by EDAX spectra and line scans over the entire catalyst in order to eliminate $\mathrm{CoS}_{2}$ as a possible secondary phase and conclude that cobalt is indeed present only as a dopant in the $\mathrm{MoS}_{2} / \mathrm{WS}_{2}$ systems studied here.

$\mathrm{MoS}_{2}$ is a layered material consisting of weakly bounded sulfurmolybdenum-sulfur sheets and a single layer can be terminated by two different edges: the molybdenum-terminated edge (1010) (Mo-edge) and the sulfur-terminated (1010) edge (S-edge). The Mo-edge exposes a row of molybdenum atoms with a coordination of only four sulfur atoms. The S-edge corresponds to an edge where the Mo atoms are fully saturated with sulfur atoms. Density functional theory calculations concentrate on the adsorption of sulfur on the molybdenum-terminated edges and the formation of vacancies on the sulfur-terminated edges to investigate the nature of the catalytic properties of $\mathrm{MoS}_{2}$ (Byskov et al., 1997; Raybaud, 2000). In the past two decades, numerous configurations for the sulfulradsorption and the cobalt-promoter distribution at the molybdenum and sulfur active edges of $\mathrm{MoS}_{2}$ have been intensively investigated by different theoretical and experimental groups. One of the most relevant density functional theory simulation results shows that, for typical HDS conditions, the energy of the S-edge with 100\% cobalt is lower than the Mo-edge with 100\% cobalt (Krebs et al., 2009; Schweiger et al., 2002; Byskov et al., 2000; Sun et al., 2004a). However, in addition to the affinity of cobalt for the S-edge under such reaction conditions, a 50\% cobalt-promoter decoration of the Mo-edge reduces considerably the edge energy of the Mo-edge to 
a value close to the S-edge value. Such a new feature had important consequences on the morphologies considered to date and opened a new landscape of possibilities on the location and nature of active sites at the edges of the $\mathrm{Co} / \mathrm{Mo} / \mathrm{S}$ phase. In particular, density functional theory calculations showed that the $50 \%$ cobalt partial occupation with the Mo-edge covered by $25 \%$ sulfur is stable. Furthermore, the simulations also indicated the existence of two configurations with such sulfur saturation that are close in energy - namely, the alternate configuration Mo-Co-Mo-Co- and the paired configuration Mo-Mo-Co-Co. Figures 4.42a and 4.42b show a perspective view of these configurations together with their respective top views of the Mo-edge. In both cases, the Mo-edge structure exhibits mixed

(a)

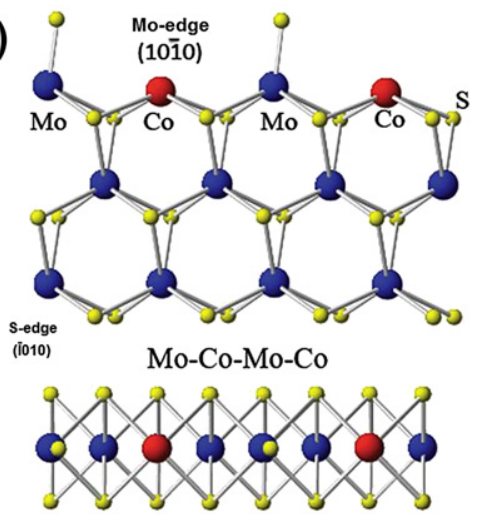

(c)

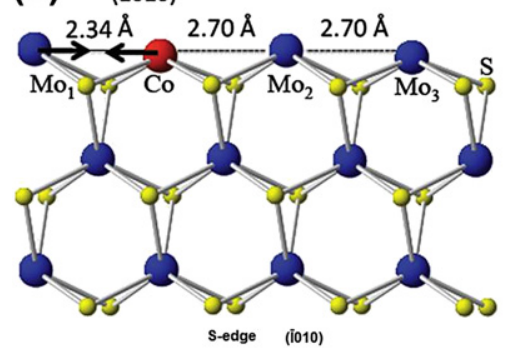

(b)

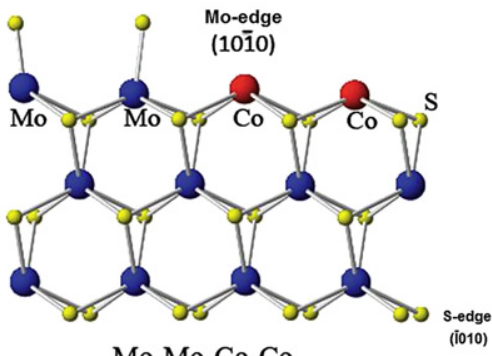

Mo-Mo-Co-Co

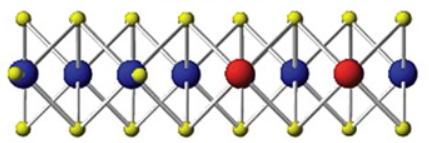

Mo-edge

(d) $\begin{gathered}\text { W.edge } \\ (10 \overline{1} 0)\end{gathered}$

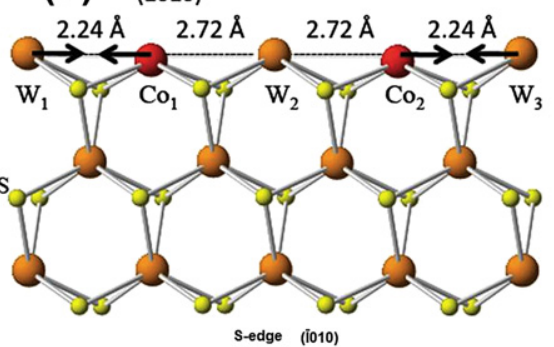

Figure 4.42 Perspective and top views of (a) Mo-edge with $50 \%$ cobalt in an alternate position and $25 \%$ sulfur. (b) Mo-edge with $50 \%$ cobalt in a pairing configuration and $25 \%$ sulfur. (c) and (d) Perspective views of CoMoS (CoWS) structure for the experimentally observed Mo-Co-Mo-Mo (W-Co-W-Co-W) sequence at the Mo/W-edge. Molybdenum atoms are in blue, tungsten ornaments are in orange, and sulfur atoms are in yellow. (Reprinted with permission from Deepak et al. (2011b). Copyright (2011) Springer). (See the color plate). 

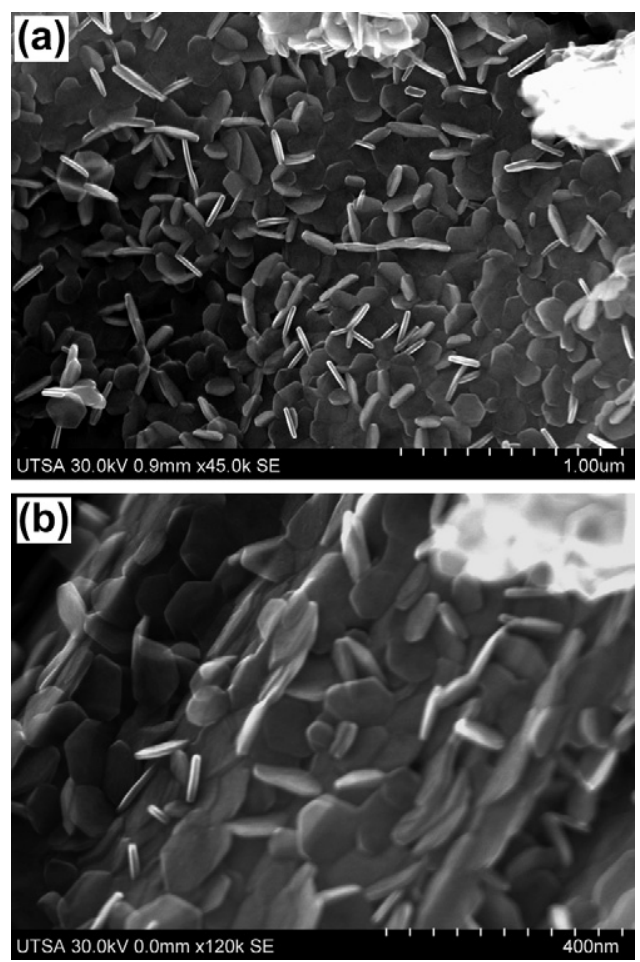

Figure 4.43 SEM micrographs of the $\mathrm{MoS}_{2}$ plates. (Reprinted with permission from Castro-Gerrero et al. (2011). Copyright (2011) Royal Society of Chemistry).

Co-Mo sites while the sulfur atom is located at the top of the molybdenum atoms. Nevertheless, it was also found that sulfur atoms might also be located in bridging positions, which indicated that the mobility of sulfur atoms on the Mo-edge is high (Krebs et al., 2009; Schweiger et al., 2002). To illustrate the experimentally observed morphologies, two models for the cobaltmolybdenum-sulfur and cobalt-tungsten-sulfur catalysts were built (see Figures $4.42 \mathrm{c}$ and $4.42 \mathrm{~d}$ ). Figure $4.42 \mathrm{c}$ shows the atomic model for the $\mathrm{MoS}_{2}$ doped with cobalt for the experimentally found -Mo-Co-Mo-Mosequence indicated previously in Figure 4.39b. In contrast to the simulations, where the optimized local cobalt-molybdenum distances are comprised between $2.74 \AA$ and $2.84 \AA$, the pairing of the Mon- and $\mathrm{Co}_{1}$-edge atoms $(2.34 \AA)$ is observed, followed by a $2.70-\AA$ separation for both $\mathrm{Co}_{1}-\mathrm{Mo}_{2}$ and $\mathrm{Mo}_{2}-\mathrm{Mo}_{3}$ atoms. Interestingly, this structure appears to be a combination of the alternate and paired configuration, which are both very close in energy. The $100 \%$ cobalt $-50 \%$ sulfur and $100 \%$ cobalt $-62.5 \%$ sulfur configuration at 
the S-edge (not shown) were not experimentally observed. Figure $4.42 \mathrm{~d}$ shows the atomic model for the cobalt-tungsten-sulfur catalysts for the $\mathrm{W}-\mathrm{Co}-\mathrm{W}-\mathrm{Co}-\mathrm{W}$ sequence presented in Figure 4.40. This model supports the idea that the cobalt-promoter atoms incorporate into the $\mathrm{MoS}_{2}$ structure by substituting Mo-edge atoms in an alternating configuration as previously mentioned. Previous density functional theory calculations indicate that under reaction conditions, $50 \% \mathrm{~S}$ coverage on the promoted $\mathrm{W}$-edge and S-edge are the most energetically stable configurations. In contrast to the CoMoS model, the sulfur atoms are located at the bridging positions according to Sun et al. (2004b). Additional density functional theory calculations are desirable to reveal in detail the sulfur-saturation configuration at the $\mathrm{W}$-edge. In general, the exact location of the sulfur atoms, and as a consequence the location of the active edges, remains an issue at the experimental level since it is not yet possible to distinguish between $25 \%$ or $50 \%$ of sulfur saturation at the Mo-edge when sulfur atoms are located on top of the Mo-edge atoms.

\section{4. $\mathrm{MoS}_{2}$ Nanoplates}

Of the various morphologies of $\mathrm{MoS}_{2}$ explored for its catalytic applications, an interesting morphology is the platelike structure. Nanohexagonal plates are particularly exciting because of the hexagonal shape and nanometric scale, which make them an ideal material in catalysis due to their high surface area. Figure 4.44 shows the SEM micrographs of the $\mathrm{MoS}_{2}$ plates synthesized at $700{ }^{\circ} \mathrm{C}$ and further annealed at $900{ }^{\circ} \mathrm{C}$ and $1000^{\circ} \mathrm{C}$. Both images show the presence of hexagonal plates with nanometric size in the sample. The size of the plates is approximately $30-35 \mathrm{~nm}$ in diameter. The agglomerates consist of individual plates stacked together.

Figure 4.44a presents an ultra-high-resolution BF-STEM image of a single $\mathrm{MoS}_{2}$ nanoplate. The Mo-Mo distances in the $\mathrm{a}_{1}, \mathrm{a}_{2}$, and $\mathrm{a}_{3}$ directions are measured as $2.76 \AA, 2.8 \AA$, and $2.87 \AA$ (the line profile is shown in the inset); these distances correspond to the Mo-Mo interatomic distances (bright atom contrast arising from the molybdenum atoms). Figure $4.44 \mathrm{~b}$ shows the electron diffraction pattern revealing the $\mathrm{a}_{1}$, $\mathrm{a}_{2}$, and $\mathrm{a}_{3}$ directions as well as the hexagonal structure of the $\mathrm{MoS}_{2}$.

Figure 4.45 shows a moire pattern formed by the superimposition of the individual plates of $\mathrm{MoS}_{2}$. The upper inset in the figure shows an HR-TEM micrograph wherein a moiré pattern of a moiré lattice is observed. The lower inset shows the fast Fourier transform (FFT) of the plates: Three sets of hexagonal spots can be seen, corresponding to the three individual plates. 

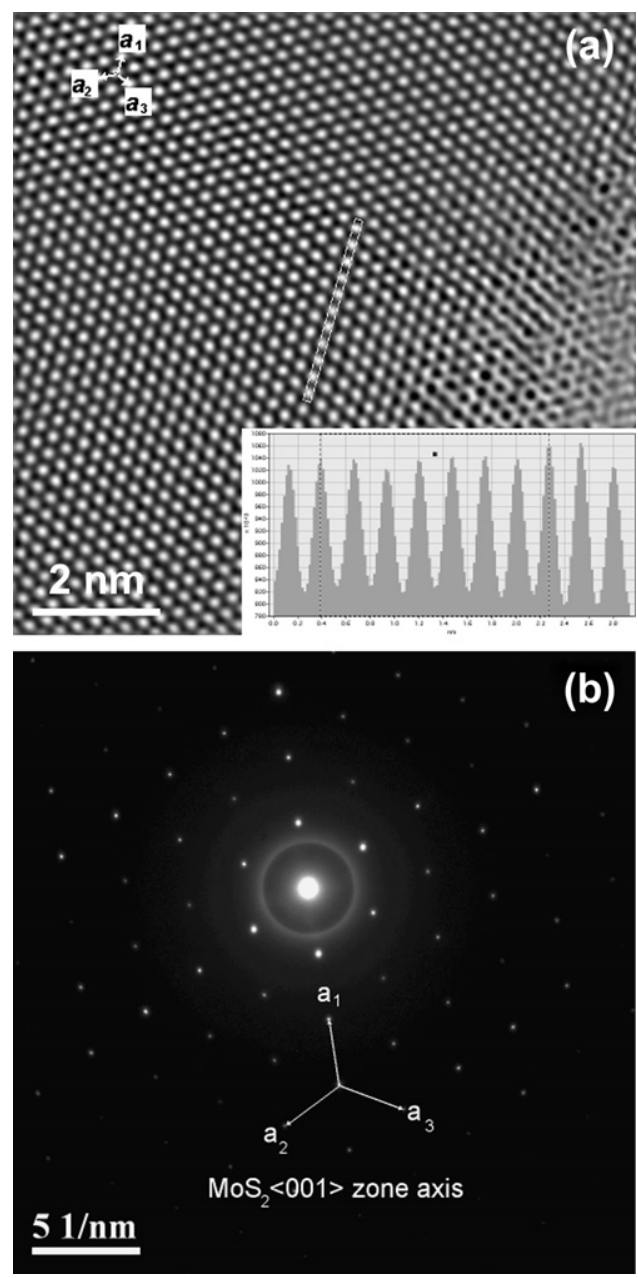

Figure 4.44 (a) BF-STEM image of a $\mathrm{MoS}_{2}$ plate (bright atom contrast shows the molybdenum atoms). The insets show the $a_{1}, a_{2}$, and $a_{3}$ directions in the sixfold zone axis as well as the measurements of the Mo-Mo interatomic distances. (b) Selected area electron diffraction pattern of the $\mathrm{MoS}_{2}$ plates showing the hexagonal symmetry in the $<001>$ direction. (Reprinted with permission from Castro-Gerrero et al. (2011). Copyright (2011) Royal Society of Chemistry).

The angles measured between the plates correspond to $19^{\circ}$ between the first two plates and $16^{\circ}$ between the next two plates; these are the rotational angles of the plates. A moire pattern is observed with the superimposition of the three plates. Moire patterns of three plates (see the boxed area, plate 3) and two plates (plate 2) are seen in the figure. 


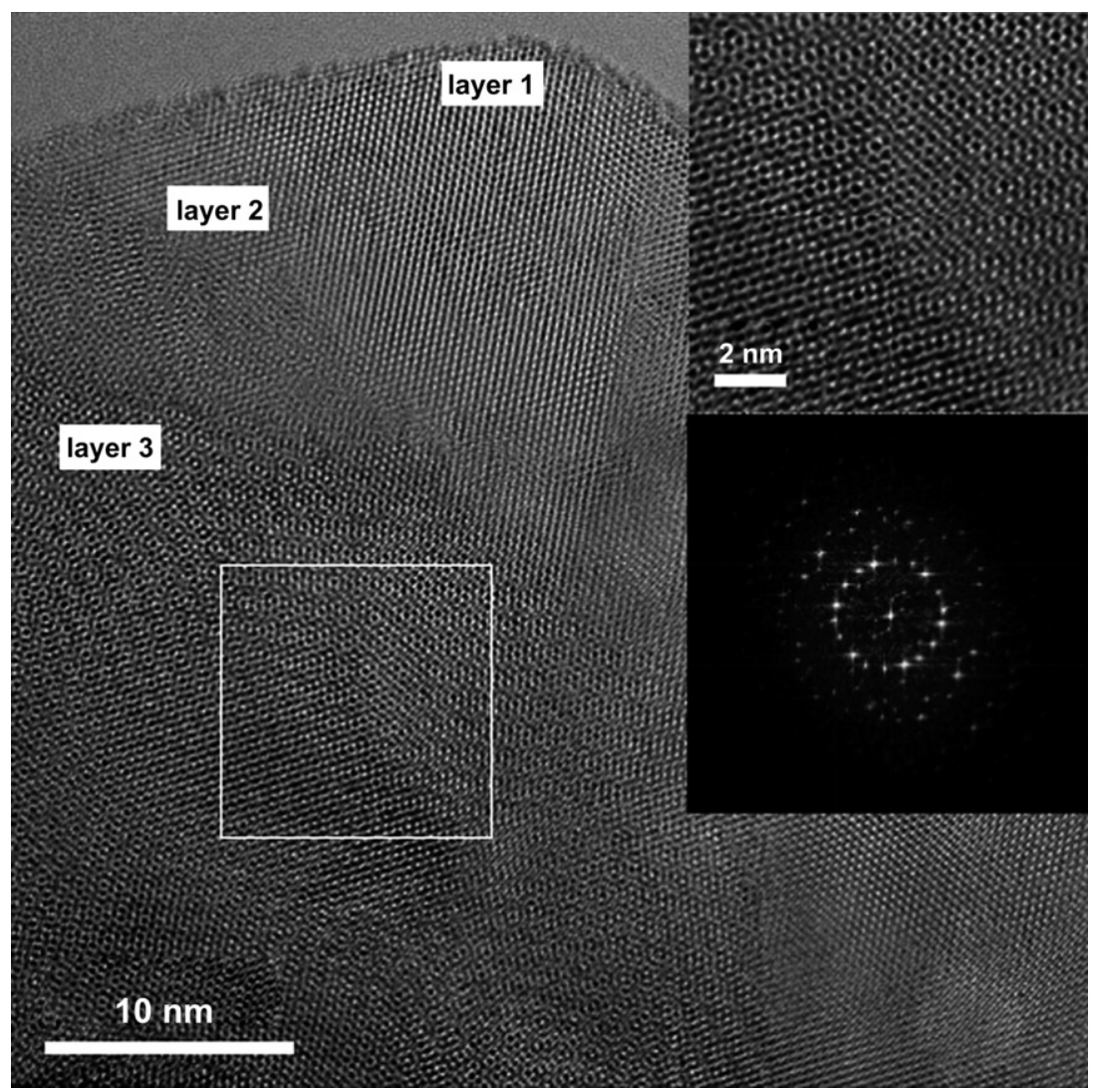

Figure 4.45 Image showing the superimposition of three plates of $\mathrm{MoS}_{2}$ and the formation of a moiré pattern. The upper inset shows moiré lattice. The lower inset shows the fast Fouriter transform pattern with three sets of spots corresponding to the three plates, the angles between the plates being $19^{\circ}$ and $16^{\circ}$. (Reprinted with permission from Castro-Gerrero et al. (2011). Copyright (2011) Royal Society of Chemistry).

Figure 4.46 shows an HR-TEM micrograph of a $\mathrm{MoS}_{2}$ plate showing a lateral view. The left inset shows the diffraction pattern revealing the direction of the crystal, which is $[000 n]$, where $n$ is even. The right inset shows an amplification of the boxed area in the main image. It Several bands of different sizes can be observed; the broad lines are molybdenum (as it has a high atomic number and a high atomic weight), whereas the thin lines are sulfur. The lines are regular and consist of alternating sulfur-molybdenumsulfur layers forming a sandwich. These sulfur-molybdenum-sulfur layers form the unit cell in $\mathrm{MoS}_{2}$ (seen in the inset). The distance measured 


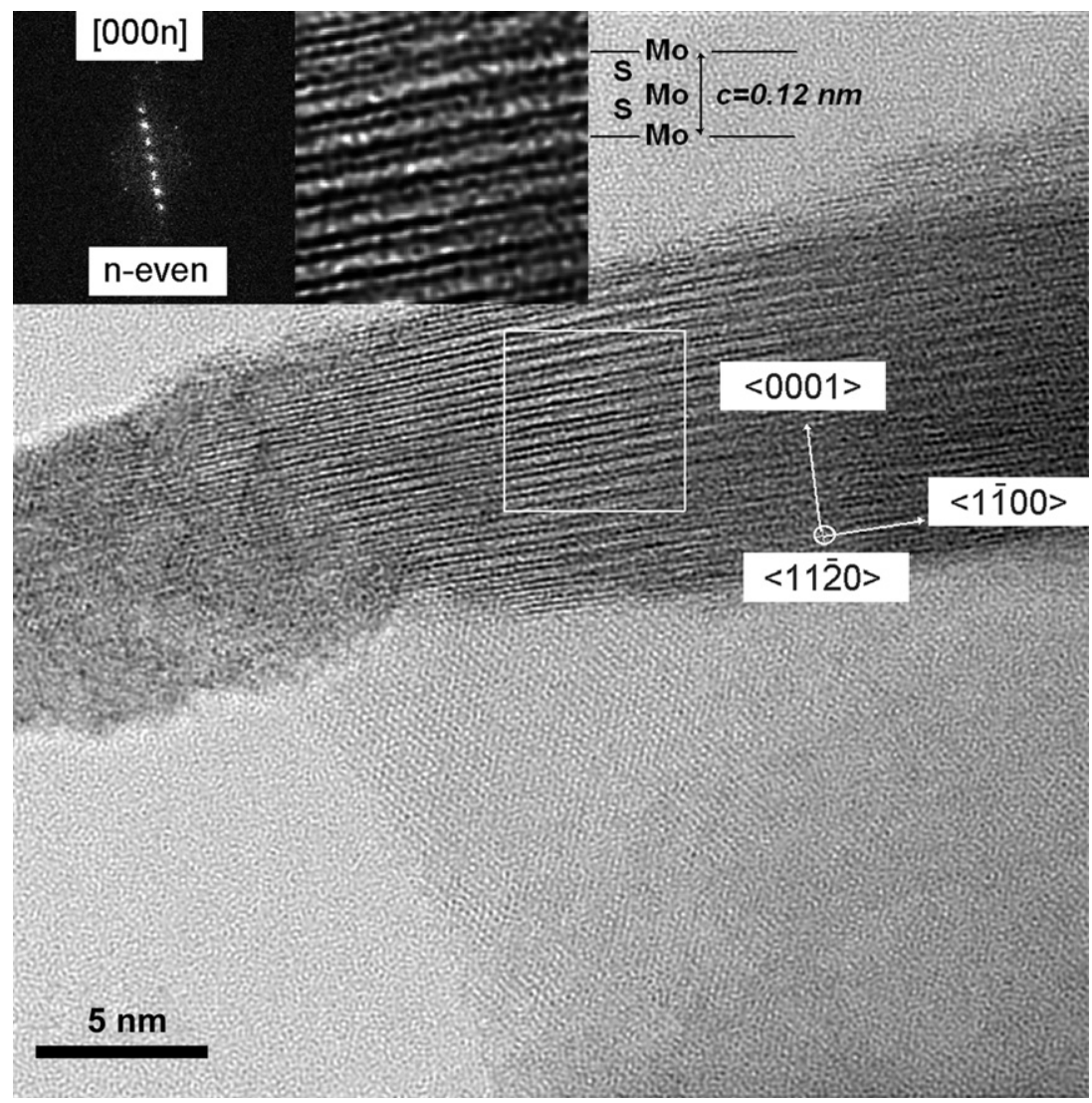

Figure 4.46 BF-STEM image of a plate showing the individual molybdenum and sulfur layers in $\mathrm{MoS}_{2}$. The left inset shows the fast Fourier transform in the $<11-20>$ zone axis, whereas the right inset shows a magnified view of the unit cell $(c=1.2 \AA)$. The individual stripes are the stacks of molybdenum and sulfur. (Reprinted with permission from Castro-Gerrero et al. (2011). Copyright (2011) Royal Society of Chemistry).

between three atoms of molybdenum forming the cell unit shows $c=1.2 \AA$, which agrees with the values obtained with $\mathrm{X}$-ray diffraction (reported value of $\mathrm{c} / 2=6.53 \AA$ ).

\subsection{Conclusions}

Elucidation of the structure and properties associated with a truly industrial catalyst such as $\mathrm{MoS}_{2}$ based on the recent advances in advanced electron microscopy promises to open new avenues in this ever-expanding area of catalysis of layered materials. Having overcome the shortcomings of the lack of understanding of structure and catalytic active sites associated with such 
catalysts before such techniques were available, the future seems quite promising in this area. Indeed, the challenges associated with solving environmental issues based on catalysis are more realistic and closer than ever before.

\section{ACKNOWLEDGMENTS}

This work was supported by grants from the National Center for Research Resources (grant No. 5 G12RR013646-12) and the National Institute on Minority Health and Health Disparities from the National Institutes of Health (grant No. G12MD007591). The authors would also like to acknowledge National Science Foundation support (DMR grant No. 1103730, "Alloys at the Nanoscale: The Case of Nanoparticles, Second Phase" and PREM grant No. 0934218, "Oxide and Metal Nanoparticles-The Interface Between Life Sciences and Physical Sciences". In addition, the authors would like to acknowledge the support of the Welch Foundation (grant No. AX-1615, "Controlling the Shape and Particles Using Wet Chemistry Methods: The Case of Bimetallic Nanoparticles", an international collaboration grant from University of California Institute for Mexico and the United States (UC MEXUS), and the Consejo Nacional de Ciencia y Tecnología (CONACYT, grant CIAM 148967). In addition, authors want to thank to Dr. Gabriela Diaz from Institute of PhysicsUNAM for provide the samples $\mathrm{Au} / \mathrm{Ir} / \mathrm{TiO} 2$ included in the present Chapter.

\section{REFERENCES}

Ahmed, F., Alam, M. K., Suzuki, A., Koyama, M., Tsuboi, H., Hatakeyama, N., Endou, A., Takaba, H., Del Carpio, C. A., Kubo, M., et al. (2009). Dynamics of hydrogen spillover on $\mathrm{Pt} / \gamma-\mathrm{Al}_{2} \mathrm{O}_{3}$ catalyst surface: a quantum chemical molecular dynamics study. Journal of Physical Chemistry C, 113(35), 15676-15683.

Allard, L. F., Bigelow, W. C., Jose-Yacaman, M., et al. (2009). A new MEMS-based system for ultra-high-resolution imaging at elevated temperatures. Microscopy Research and Technique, 72, 208-215.

Babich, I. V., \& Moulijn, J. A. (2003). Science and technology of novel processes for deep desulfurization of oil refinery streams: a review. Fuel, 82(6), 607-631.

Botello-Mendez, A. R., Lopez-Urias, F., Terrones, M., et al. (2009). Metallic and ferromagnetic edges in molybdenum disulfide nanoribbons. Nanotechnology, 20(32), 1-6.

Brorson, M., Carlsson, A., \& Topsoe, H. (2007). The morphology of $\mathrm{MoS}_{2}, \mathrm{WS}_{2}, \mathrm{Co}-$ Mo-S, Ni-Mo-S and Ni-W-S nanoclusters in hydrodesulfurization catalysts revealed by HAADF-STEM. Catalysis Today, 123(1-4), 31-36.

Byskov, L. S., Hammer, B., Nørskov, J. L., et al. (1997). Sulfur bonding in $\mathrm{MoS}_{2}$ and CoMo-S structures. Catalysis Letters, 47(3-4), 177-182.

Byskov, L. S., Nørskov, J. K., Clausen, B. S., et al. (1999). DFT calculations of unpromoted and promoted $\mathrm{MoS}_{2}$-based hydrodesulfurization catalysts. Journal of Catalysis, 187(1), 109-122.

Byskov, L. S., Nørskov, J. K., Clausen, B. S., et al. (2000). Edge termination of $\mathrm{MoS}_{2}$ and CoMoS catalyst particles. Catalysis Letters, 64(2-4), 95-99.

Carter, C. B., \& Williams, D. B. (2009). Transmission Electron Microscopy: A Textbook for Materials Science. New York: Springer.

Castro-Guerrero, C. F., Deepak, F. L., Ponce, A., et al. (2011). Structure and catalytic properties of hexagonal molybdenum disulfide nanoplates. Catalysis Science \& Technology, 1, 1024-1031. 
Chen, J., Kuriyama, N., Yuan, H., et al. (2001). Electrochemical hydrogen storage in $\mathrm{MoS}_{2}$ nanotubes. Journal of the American Chemical Society, 123(47), 11813-11814.

Corthey, G., Giovanetti, L. J., Ramallo-López, J. M., et al. (2010). Synthesis and characterization of gold at gold(i)-thiomalate core at shell nanoparticles. ACS Nano, 4, 3413-3421.

Deepak, F. L., Casillas-Garcia, G., Esparza, R., et al. (2011a). New insights into the structure of Pd-Au nanoparticles as revealed by aberration-corrected STEM. Journal of Crystal Growth, 325(1), 60-67.

Deepak, F. L., Esparza, R., \& Borges, B. (2011b). Rippled and helical $\mathrm{MoS}_{2}$ nanowire catalysts: an aberration corrected STEM study. Catalysis Letters, 141(4), 518-524.

Deepak, F. L., Esparza, R., Borges, B., et al. (2011c). Direct imaging and identification of individual dopant atoms in $\mathrm{MoS}_{2}$ and $\mathrm{WS}_{2}$ catalysts by aberration corrected. Scanning transmission electron microscopy. ACS Catalysis, 1(5), 537-543.

Dhas, N. A., \& Suslick, K. S. (2005). Sonochemical preparation of hollow nanospheres and hollow nanocrystals. Journal of American Chemical Society, 127(8), 2368-2369.

Diebol, U. (2003). The surface science of titanium dioxide. Surface Science Reports, 48(5), 53-229.

Ding, Y., Fan, F., Tian, Z., et al. (2010). Atomic structure of Au-Pd bimetallic alloyed nanoparticles. Journal of American Chemical Society, 132(35), 12480-12486.

Ding, Y., Sun, X., Wang, Z. L., et al. (2012). Misfit dislocations in multimetallic coreshelled nanoparticles. Applied Physics Letters, 100(11), 111603.

Escalona, E. E., Pereira-Almao, P. R., Castillo, J., et al. (2006). Nanometric bimetallic sulfides prepared via thermal decomposition of $\mathrm{Ni}$ and $\mathrm{Fe}$ heteropolymolybdate emulsions. Catalysis Letters, 112(3-4), 227-230.

Farraghher, A. L. (1979). Surface vacancies in close packed crystal structures. Advances in Colloid and Interface Science, 11(1), 3-41.

Ferrer, D., Blom, D. A., Allard, L. F., et al. (2008). Atomic structure of three-layer Au/Pd nanoparticles revealed by aberration-corrected scanning transmission electron microscopy. Journal of Materials Chemistry, 18, 2442-2446.

Ferrer, D. A., Diaz-Torres, L. A., Wu, S., et al. (2009). Crystalline order of silvergold nanocatalysts with hollow-core and alloyed-shell. Catalysis Today, 147(3), 211-216.

Frenkel, A. I., Hills, C. W., \& Nuzzo, R. G. (2001). A view from the inside: complexity in the atomic scale ordering of supported metal nanoparticles. Journal of Physical Chemistry B, 105(51). 15689-12703.

Frey, G. L., Tenne, R., Matthews, M. J., et al. (1999). Raman and resonance Raman investigation of $\mathrm{MoS}_{2}$ nanoparticles. Physical Review B, 60, 2883-2892.

Friel, J. J., \& Lyman, C. E. (2006). Tutorial review: X-ray mapping in electron-beam instruments. Microscopy and Microanalysis, 12(1), 2-25.

Galvan, D. H., Deepak, F. L., Esparza, R., et al. (2011). Experimental and theoretical properties of S-Mo-Co-S clusters. Applied Catalysis A: General, 397(1), 46-53.

Gates, B. C. (1992). Catalytic Chemistry. New York: Wiley.

Hansen, L. P., Ramasse, Q. M., Kisielowski, C., et al. (2011). Atomic-scale edge structures on industrial-style $\operatorname{mos}_{2}$ nanocatalysts. Angewandte Chemie International Edition, 50(43), 10153-10156.

Harada, H., Asakura, K., \& Toshima, N. (1993). Catalytic Activity and Structural Analysis of Polymer-Protected $\mathrm{Au} / \mathrm{Pd}$ Bimetallic Clusters Prepared by the Successive Reduction of HAuC14 and PdCl2. Journal of Physical Chemistry, 97(19), 5103-5114.

He, J., Ichinose, I., Kunitake, T., et al. (2003). Facile fabrication of Ag-Pd bimetallic nanoparticles in ultrathin $\mathrm{TiO}_{2}$-gel films: nanoparticle morphology and catalytic activity. Journal of the American Chemical Society, 125(36), 11034-11040. 
Homyonfer, M., Alperson, B., Rosenberg, Y., et al. (1997). Intercalation of inorganic fullerene-like structures yields photosensitive films and new tips for scanning probe microscopy. Journal of American Chemical Society, 119(11), 2693-2698.

Iglic, A., Tzaphlidou, M., Remskar, M., et al. (2005). Stable shapes of thin anisotropic nanostrips. Fullerenes Nanotubes Carbon Nanostructures, 13(3), 183-192.

Ionescu, A., Allouche, A., Aycard, J. P., et al. (2003). Study of $\gamma$-alumina-supported hydrotreating catalyst: I. Adsorption of bare $\mathrm{MoS}_{2}$ sheets on $\gamma$-alumina surfaces. Journal of Physical Chemistry B, 107(33), 8490-8497.

Jang, Y. B., Kim, T. H., Sun, M. H., et al. (2009). Preparation of iridium catalyst and its catalytic activity over hydrazine hydrate decomposition for hydrogen production and storage. Catalysis Today, 146, 196-201.

Kisielowski, C., Ramasse, Q. M., Hansen, L. P., et al. (2011). Imaging $\mathrm{MoS}_{2}$ nanocatalysts with single-atom sensitivity. Angewandte Chemie International Edition, 49(15), 27082710.

Koch, C. T. (2002). Determination of core structure periodicity and point defect density along dislocations. Pro Quest Dissertations and Theses; Ph.D. thesis. Arizona State University.

Kotaka, Y. (2010). Essential experimental parameters for quantitative structure analysis using spherical aberration-corrected HAADF-STEM. Ultramicroscopy, 110(5), 555-562.

Kralj-Iglic, V., Remskar, M., \& Iglic, A. (2004). Horizons in World Physics. New York: Nova Science Publishers. 111-156.

Krebs, E., Daudin, A., \& Raybaud, P. (2009). A DFT study of CoMoS and NiMoS catalysts: from nano-crystallite morphology to selective hydrodesulfurization. Oil \& Gas Science and Technology, 64(6), 707-718.

Li, Y., Zhou, Z., Zhang, S., et al. (2008). $\mathrm{MoS}_{2}$ Nanoribbons: High Stability and Unusual Electronic and Magnetic Properties. Journal of the American Chemical Society, 130, 1673916744.

Li, Y. W., \& Delmon, B. (1997). Modelling of hydrotreating catalysis based on the remote control: HYD and HDS. Journal of Molecular Catalysis A: Chemical, 127(1-3), 163-190.

Lince, J. R. (2004). Tribology of Co-sputtered nanocomposite $\mathrm{Au} / \mathrm{MoS}_{2}$ solid lubricant films over a wide contact stress range. Tribology Letters, 17(3), 419-428.

Longo, R. C., \& Gallego, L. J. (2006). Structures of 13-atom clusters of fcc transition metals by ab initio and semiempirical calculation. Physical Review B, 74, 193409.

Mayoral, A., Barron, H., Estrada-Salas, R., et al. (2010). Nanoparticle stability from the nano to the meso interval. Nanoscale, 2, 335-342.

Mayoral, A., Esparza, R., Deepak, F. L., et al. (2011). Study of nanoparticles at UTSA: one year of using the first JEM-ARM200F installed in USA. JEOL News, 46, 1-5.

Mazumder, V., Chi, M., More, K. L., et al. (2010). Synthesis and characterization of multimetallic $\mathrm{Pd} / \mathrm{Au}$ and $\mathrm{Pd} / \mathrm{Au} / \mathrm{FePt}$ core/shell nanoparticles. Angewandte Chemie International Edition, 49(49), 9368-9372.

Mejia-Rosales, S., Fernandez-Navarro, C., Perez-Tijerina, E., et al. (2006). Molecular dynamics runs to simulate the melting transition of bimetallic cuboctahedral nanoparticles of gold palladium. Journal of Physical Chemistry B, 110(26), 12884-12889.

Mizukoshi, Y., Fujimoto, T., Nagata, Y., et al. (2000). Characterization and catalytic activity of core-shell structured gold/palladium bimetallic nanoparticles synthesized by the sonochemical method. Journal of Physical Chemistry B, 104(25), 6028-6032.

Olivas, A., Alonso, G., \& Fuentes, S. (2006). The catalytic activity of Ni/W bimetallic sulfide nanostructured catalysts in the hydrodesulfurization of dibenzothiophene. Topics in Catalysis, 39(3-4), 175-179.

Pennycook, S. (1989). Z-contrast STEM for materials science. Ultramicroscopy, 30, 58-69.

Pennycook, S. J., \& Boatner, L. A. (1998). Chemically sensitive structure-imaging with a scanning transmission electron microscope. Nature, 336, 565-567. 
Perez-Tijerina, E., Mejia-Rosales, S., Inada, H., et al. (2010). Effect of temperature on AuPd nanoparticles produced by inert gas condensation. Journal of Physical Chemistry C, 114(15), 6999-7003.

Perez-Tijerina, E., Pinilla, G. M., Mejia-Rosales, S., et al. (2008). Highly size-controlled synthesis of $\mathrm{Au} / \mathrm{Pd}$ nanoparticles by inert-gas condensation. Faraday Discussions, 138, 353-362.

Raybaud, P., Hafner, J., Kresse, G., et al. (2000). Structure, energetics, and electronic properties of the surface of a promoted $\mathrm{MoS}_{2}$ catalyst: an ab initio local density functional study. Journal of Catalysis, 190(1), 128-143.

Recnik, A., Möbus, G., \& Sturm, S. (2005). IMAGE-WARP: a real-space restoration method for high-resolution STEM images using quantitative HRTEM analysis. Ultramicroscopy, 103(4), 285-301.

Reimer, L., \& Kohl, H. (2008). Transmission Electron Microscopy: Physics of Image Formation. New York: Springer.

Remskar, M., Skraba, Z., Stadelmann, P., et al. (2000). Structural stabilization of new compounds: $\mathrm{MoS}_{2}$ and $\mathrm{WS}_{2}$ micro- and nanotubes alloyed with gold and silver. Advanced Materials, 12(11), 814-818.

Rioux, R. M., Song, H., Grass, M., et al. (2006). Monodisperse platinum nanoparticles of well-defined shape: synthesis, characterization, catalytic properties and future prospects. Topics in Catalysis, 39(3), 167-174.

Rose, H. (1990). Outline of a spherically corrected semiaplanatic medium-voltage transmission electron microscope. Optik, 85, 19-24.

Schweiger, H., Raybaud, P., \& Toulhoat, H. (2002). Promoter sensitive shapes of Co(Ni) MoS nanocatalysts in sulfo-reductive conditions. Journal of Catalysis, 212(1), 33-38.

Senevirathne, K., Burns, A. W., Bussell, M. E., et al. (2007). Synthesis and characterization of discrete nickel phosphide nanoparticles: effect of surface ligation chemistry on catalytic hydrodesulfurization of thiophene. Advanced Functional Materials, 17(18), 3933-3939.

Shidpour, R., \& Manteghian, M. (2010). A density functional study of strong local magnetism creation on $\mathrm{MoS}_{2}$ nanoribbon by sulfur vacancy. Nanoscale, 2(8), 1429-1435.

Sinfelt, J. H. (1983). Bimetallic Catalyst: Discoveries, Concepts and Applications. New York: Wiley.

Song, C. (2003). An overview of new approaches to deep desulfurization for ultra-clean gasoline, diesel fuel and jet fuel. Catalysis Today, 86(1-4), 211-263.

Srnová-Šloufová, I., Lednický, F., Gemperle, A., \& Gemperlová, J. (2000). Core-shell (Ag) $\mathrm{Au}$ bimetallic nanoparticles: analysis of transmission electron microscopy images. Langmuir, 16(25), 9928-9935.

Stamenkovic, V. R., Fowler, B., Mun, B. S., et al. (2007). Improved oxygen reduction activity on $\mathrm{Pt} 3 \mathrm{Ni}(111)$ via increased surface site availability. Science, 315(5811), 493-497.

Stroppa, D. G., Zagonel, L. F., Montoro, L. A., et al. (2012). High-resolution scanning transmission electron microscopy (HRSTEM) techniques: high-resolution imaging and spectroscopy side by side. ChemPhysChem, 13(2), 437-443.

Sun, M., Nelson, A. E., \& Adjaye, J. (2004a). On the incorporation of nickel and cobalt into $\mathrm{MoS}_{2}$-edge structures. Journal of Catalysis, 226(1), 32-40.

Sun, M., Nelson, A. E., \& Adjaye, J., J. (2004b). A DFT study of $\mathrm{WS}_{2}$, NiWS, and CoWS hydrotreating catalysts: energetics and surface structures. Journal of Catalysis, 226(1), 41-53.

Topsøe, H. (2007). The role of $\mathrm{Co}-\mathrm{Mo}-\mathrm{S}$ type structures in hydrotreating catalysts. Applied Catalysis A, General, 322, 3-8.

Toshima, N. (1996). Fine Particles Science and Technology: From Micro to Nanoparticles. In E. Pellizzetti (Ed.), Colloidal Dispersion of Bimetallic Nanoparticles: Preparation, Structure and Catalysis (pp. 371-383). Dordrecht: Kluwer. 
Turaga, U. T., Ma, X., \& Song, C. S. (2003). Influence of nitrogen compounds on deep hydrodesulfurization of 4,6-dimethyldibenzothiophene over $\mathrm{Al}_{2} \mathrm{O}_{3}$ - and $\mathrm{MCM}-$ 41-supported Co-Mo sulfide catalysts. Catalysis Today, 86(1-4), 265-275.

Tye, C. T., \& Smith, K. J. (2004). Catalytic activity of exfoliated $\mathrm{MoS}_{2}$ in hydrodesulfurization, hydrodenitrogenation and hydrogenation reactions. Topics in Catalysis, 37(2-4), 129-135.

Wall, J., Langmore, J., Isaacson, M., et al. (1974). Scanning transmission electron microscopy at high resolution. Proceedings of the National Academy of Sciences, 71, 1-5.

Whitehurst, D. D., Isoda, T., \& Mochida, I. (1998). Present state of the art and future challenges in the hydrodesulfurization of polyaromatic sulfur compounds. Advances in Catalysis, 42, 345-471.

Wu, Z., Wang, D., Liang, X., et al. (2010). Novel hexagonal $\mathrm{MoS}_{2}$ nanoplates formed by solid-state assembly of nanosheets. Journal of Crystal Growth, 312(12-13), 1973-1976.

Yoosuk, B., Kim, J. H., Song, C., et al. (2008). Highly active $\mathrm{MoS}_{2}, \mathrm{CoMoS}_{2}$ and $\mathrm{NiMoS}_{2}$ unsupported catalysts prepared by hydrothermal synthesis for hydrodesulfurization of 4,6-dimethyldibenzothiophene. Catalysis Today, 130(1), 14-23.

Zak, Z., Feldman, Y., Lyakhovitskaya, V., et al. (2002). Alkali metal intercalated fullerenelike $\mathrm{MS}_{2}(\mathrm{M}=\mathrm{W}, \mathrm{Mo})$ nanoparticles and their properties. Journal of the American Chemical Society, 124(17), 4747-4758.

Zuo, J. M. (2008). JProbe. Available at. http://cbed.matse.illinois.edu/. 


\section{INDEX}

Note: Page numbers followed by " $\mathrm{f}$ " and " $\mathrm{t}$ " indicate figures and tables, respectively

\section{A}

Aberrations, 195-196, 287-290 contrast transfer function (CTF), 287 optical aberrations, 196-197

Absolute convergence, 63-64

Accelerator system, 262

ADF. See Annular dark-field

Adjoint dilation, 92

Adjoint erosion, 92

Adjoint signal dilation, 128

Adjoint signal erosion, 127

Adjunction, 52-53, 92

flat erosion, 94

Minkowski flat dilation, 94

Minkowski set dilation, 93

Minkowski set erosion, 94

Minkowski weighted erosion, 95

properties, 93

real-valued functions, 94

supremal convolution, 95

Algebraic decomposition, 72

algebraic complement, 72

linear space, 72

Algebraic isomorphism, 71

Alternating sequential filter (ASF), 108-109

Amoeba filters, 97

Annular dark-field (ADF), 283, 285-286

Anti-isomorphism. See Dual isomorphism

Antitone. See Decreasing operators

Area filters

area opening, 104-105

attribute openings, 105-106

ASF. See Alternating sequential filter

Atom, 82

B

Banach space, 63

Bas $(\psi), 157$

Benzécri algorithm, 17. See also

Two-dimensional algorithm

(2D algorithm) image size, 18

Pseudo-code, $18 \mathrm{f}$

Benzécri HAC. See Benzécri hierarchical ascendant classification

Benzécri hierarchical ascendant classification (Benzécri HAC), 14-15

hierarchy

dissimilarity index, 16

parts and levels, 15

property, 15

using remarks, 16

segmentations, 15

stratified hierarchy, 16

image segmentation, 15

using 2D algorithm, 15

2D images, 17

ultrametrics, 16

circle's diameter, 17

properties, 16-17

ultra metric, 17

ultrametrical space, 17

BF images. See Bright-field images

Bimetallic nanoparticles, 304

using advanced electron microscopic techniques

aberration-corrected STEM images, $307 \mathrm{f}$

$\mathrm{Au}_{1} \mathrm{Pd}_{4}$ nanoparticle, $312,313 \mathrm{f}$

BF-STEM images, 312-314, 314f

characterization, $308,308 \mathrm{f}$

chemical maps, $309 \mathrm{f}$

clusters of opalladium-iridium,

315-316, $315 f$

EDAX, 307-308

EELS map, 309, $310 \mathrm{f}$

factors, 306-307

four-layer $\mathrm{Au} / \mathrm{Pd}$ nanoparticles,

309-311, 311f

gold-palladium, 306-307

HAADF and BF-STEM images, 314, $315 f$

HAADF images, $306 f$ 
Bimetallic nanoparticles (Continued)

using HR-TEM, 305

NBD, 311-312, 312f

palladium core, 307-308

structures, 305, 305f

three-layer nanoparticle, 309, $310 \mathrm{f}$

in situ heating experiments, 316

HAADF and BF image pairs, $317 \mathrm{f}$

Ostwald ripening effects, 316-318

palladium-gold-palladium nanoparticles, 316-318

Boolean algebra. See Boolean lattices

Boolean filter, 162-163

Boolean functions

binary filter, 163

binary indicator function, 162-163

binary signal, 163-164

increasing functions, 162

minimal true and maximal false vector, 162, 164

representation theory, 161-162

stack filters, 164

Boolean lattices, 83

Boolean algebra, 83

Boolean functions, 84

field of sets, 83

finite, 83

n-dimensional Boolean vectors, 83-84

Boolean operator. See Boolean filter

Bounded linear operators space, 69-70

Bright-field images (BF images), 283

British Thomson Houston Co. (BTH), 191

\section{C}

Cambridge high-voltage microscope project accelerator system, 262

acceptance tests and delivery, 263-264

bulky item cases, 264

forming process, 265-266

high-voltage set, 267

Minkner and Bach, 266-267

Reinhold report, 264

Cavendish, 245-246

Cockcroft-Walton generator, 247-248

construction work, 247

Cosslett's plan, 247

departure from Cavendish
EM group, 270-271

HVEM, 269-270

Professor Ratcliffe, 268-269

ramifications, 269

reminiscences, 271

William Du Bois Duddell Medal, 271f

electron lenses, 263

first test report, 267-268

micrographs, $269 f$

using forklift trolley, 252-253

freighter aircraft, 248

Haefely equipment, 250

high-voltage generator, 247

high-voltage room

aluminum panels, 259

annual garden party, 262

finished and fully operational, $265 \mathrm{f}$

Millpool, 261-262

normal tea-room hum conversation, 261

Ray Dolby, 259-261

Ron Pryor and Richard Donoghay polishing, 260f

injector system, 262

microscope complex, $254 \mathrm{f}$

operating an electron microscope, 246

Paul Instrument Fund Committee, 249

radiation protection, 253

repairs to Whitegates, 250-251

CEGB, 251

high-voltage set, $251 \mathrm{f}$

after removing cable and rethatching, $252 \mathrm{f}$

showing electricity cable strung, $252 \mathrm{f}$

structural alterations

emergency exit, $256 \mathrm{f}$

establishment, 258-259

face-to-face meeting, 255

high-voltage power transmission, 256-257

installing earthing rods, $255 \mathrm{f}$

magnetic shielding, 257

sightseeing, 257-258

university estate management service, 254-255

vibration-isolating block, 253-254

vibration transmission, 253 
X-ray microscope, 249-250

Cambridge Western, 274

Carbon fullerene onion (CNO), 299

Catalyst characterization

bimetallic nanoparticles, 304

using advanced electron microscopic techniques, 305-316

structure, 305-316

clusters, 304

metal catalysts

on $\gamma-\mathrm{Al}_{2} \mathrm{O}_{2}, 301-304$

on crystalline substrate, 294-299

on noncrystalline substrate, 299-301

multimetallic systems, 318-319

in situ heating experiments

bimetallic nanoparticles, 316

clusters, 316-318

Catalytic systems, 280

Cauchy sequence, 63

Cauchy-Schwarz inequality, 64

CBED. See Convergent beam electronic diffraction

Chain, 78, 81

Chapman, A. H., 213

Charles Oatley, EM at Cambridge University. See also Ellis Cosslett, EM at Cambridge University

applications, 219-220

etched germanium surface, $220 \mathrm{f}$

plastic scintillator, 221

PPRIC, 222

sailing, 223-224

silver azide crystals, 222-223

silver azide needle, $223 \mathrm{f}$

spruce wood fiber, $222 \mathrm{f}$

TEM, 220-221

informal contacts

conversation, 203

sanatron, 204

personal affairs, 205

at Cambridge, 205-206

fairwell to crew, 206-207

Sheila, motorcycling, $205 \mathrm{f}$

time for music, 208-209

personal interlude, 230-231

Alvis Speed 20, $230 \mathrm{f}$

announcement, 232
Cambridge in one day's driving,

236-237

journey, 231

magical experience, 234

old fisherman posing, 235f

Peggy McLean, 234-236, $235 f$

Pru Woodman, 233, 233f

stomach-churning experience, 231

SU carburetors, 231f

problems with microscope, 209

electron beam, 210-211

elementary error cost, 211

frustrating experience, 210

Oliver Wells and author confer, 211f

SEM, 214

SEM3, 213-214

stigmator, 210

wireless receivers, 212

scanning electron microscope

detector system, 197

electron optical projects, 200-201

electron spot, 196

electron trajectory tracer, 203

Ellis Cosslett's electron microscopy, $202 \mathrm{f}$

improvement in efficiency, 199

limitation, 194-195

magnification, 196

McMullan's original incarnation, $198 \mathrm{f}$ practical electronics work, 198

principles, 195-196

proceedings, 202

schematic diagram, $195 \mathrm{f}$

Sheila Smith at controls, 201f

technical problems, 199

TEM invention, 193-194

transmission microscope, 193

visual acuity, 202

SEM1, 224-225, 225f

SEM2, 225

SEM3, 226, 228f

AEI, 226

construction, 228-230

cross section, $229 \mathrm{f}$

electromagnetic lenses, 226

EM4, 226-227

Everhart-Thornley detector, 227-228 
Charles Oatley, EM at Cambridge

University (Continued)

for export to Canada, 237

"KCA passed this way," $238 \mathrm{f}$

preparation and manipulation, 227

sumptuous first-class fare, 237-239

specimen chamber, 214-215, $216 f$

applications, 216

Glasgow conference, 217

Oliver in diving gear, $218 \mathrm{f}$

point-contact rectifiers, 215

publication, 216

SEM, 217

testing, 215-216

weather, 219

Clodum, 56-57, 110

bounded lattice-ordered group, 113-114

dual addition, 112

dual multiplication, 112

lattice arithmetic algebraic structure,

112-113

nonlinear spaces, 114-119

complete weighted lattices of function, 118-119

general algebraic structure, 114-116

sup/inf span, independence, basis, dimension, 116-117

self-conjugate element, 114

Clusters, 304

in situ heating experiments, 316

HAADF and BF image pairs, $317 \mathrm{f}$

Ostwald ripening effects, 316-318

palladium-gold-palladium nanoparticles, 316-318

of opalladium-iridium, 315-316, 315f

CNO. See Carbon fullerene onion

CNR. See French National Centre for Scientific Research

Co-doped $\mathrm{MoS}_{2} / \mathrm{WS}_{2}$ nanowire catalysts, 326-327

BF-STEM and HAADF-STEM images, 327f, $328 \mathrm{f}$

density functional theory calculations, 331-334

EDAX line scans, 330-331, 331f

high-resolution, 329, 330f

low-magnification, 327-329, $329 \mathrm{f}$ perspective and top views, $332 \mathrm{f}$

SEM micrographs, $333 \mathrm{f}$

Cockcroft-Walton generator, 247-248

Complete weighted lattice space (CWL space), 114-115

image operators on, 119

signal translations, 123

Complex linear space. See Hilbert space;

Real linear space

Computational theory, 49

Condenser system, 194

Connected filters, 104

Continuous functions, 67

Contrast transfer function (CTF), 287

Conventional electron microscope, 194

Conventional transmission electron microscopy (CTEM), 283

\section{Convergence}

continuity on topological image spaces, 98

hit-miss topology, 98

image signal space, 99

set sequence, 98-99

u.s.c., 99

order convergence

continuity on lattices, 99

erosions and dilations, 101

limsup and liminf combinations, 100

minimax inequality, 100

monotonic convergence, 100

order continuity, 100

sequence, 63

Convergent beam electronic diffraction

(CBED), 293

Convex analysis and optimization, 54

digital geometry, 54

Legendre-Fenchel conjugate transform, 54

Convolution adjunctions, scalar dilations, 127

Cosslett, Ellis

AEI EM7 lenses, 273

EM7, 272

Institute of Electron Optics, 272-273

John Catto's contribution, 273-274

start-up companies, 274

Cambridge Western, 274 
Granta Electronics, 274

SDI, 275

synoptics origins, 275

Whitegates again, 276

Coventry City boundary, 191

CTEM. See Conventional transmission electron microscopy

CTF. See Contrast transfer function

CWL space. See Complete weighted lattice space

\section{D}

Dark-field mode (DF mode), 283

DBT. See Dibenzothiophene

Decreasing operators, 90

class, 92

anti-dilations, 92

anti-erosions, 92

dilations, 92

erosions, 92

complete lattices, 91

dual homomorphisms, 90

dual isomorphism, 91

grey-level images, 91

signal operators, 91

thresholding operators, 91

DEDS. See Discrete-event systems control

Definite matrix, 144

Detector system, 197

DF mode. See Dark-field mode

DFT. See Discrete Fourier transform

Dibenzothiophene (DBT), 319-320

Digital geometry, 54

Digital image processing, 49

Dilation translation-invariant system (DTI system), 126

Dilation V-translation invariant operator (DVI operator), 122

Dilations, 48

4,6-Dimethyldibenzothiophene (4, 6-DMDBT), 319-320

Discrete Fourier transform (DFT), 298-299

Discrete linear filter, 167

Discrete space. See Euclidean space

Discrete version. See Euclidean domain

Discrete-event systems control (DEDS), 55

Dissimilarity index, 16
Distance transform, 54

Distributive lattice, $79-80$

4,6-DMDBT. See 4,6-

Dimethyldibenzothiophene

DTI system. See Dilation translationinvariant system

Dual atoms, 82

Dual automorphism, 79

Dual isomorphism, 91

Dual poset, 78

Dual semi-atoms, 82

Duality principle, 78

DVI operator. See Dilation V-translation invariant operator

\section{E}

ED. See Electron diffraction

EDAX. See Energy dispersive analysis of $\mathrm{X}$-ray

EDS. See Energy-dispersive spectroscopy

EDX spectroscopy. See Energy-dispersive $\mathrm{X}$-ray spectroscopy

EELS. See Electron energy-loss spectroscopy

Eigennode, 144

Eigenvalue, 144

Eigenvectors, 144-145

Electron diffraction (ED), 283

Electron energy-loss spectroscopy (EELS), 283

Electron lenses, 263

Electron microscope 3 (EM3), 213-214

Electron microscope 7 (EM7), 272

Electron trajectory tracer, 203

Ellis Cosslett, EM at Cambridge University with

Cambridge high-voltage microscope project, 245-246 acceptance tests and delivery, 263-268 departure from Cavendish, 268-271 design concepts, 252-253 electron lenses, 263 first test report, 267-268 high-voltage room, 259-262 injector and accelerator system, 262 repairs to Whitegates, 250-251 structural alterations, 253-259 post in Cavendish laboratory, 239 
Ellis Cosslett, EM at Cambridge University with (Continued)

arrangements, 239-240

British Airways Vickers Viscount turboprop, 239

cash transaction, 242

commercial side, 245

convenience and flexibility, 240

double garage, 241

encyclopedia, 244

entrance to Whitegates, 240-241

image intensifiers, 243

interim measure, 244

modern microscopy, 243

Tiplady's, 245

EM3. See Electron microscope 3

EM7. See Electron microscope 7

Emile Haefely \& Co., 255-256

Energy dispersive analysis of X-ray (EDAX), 307-308

Energy-dispersive spectroscopy (EDS), 285-286

Energy-dispersive X-ray spectroscopy (EDX spectroscopy), 283

Erosion translation-invariant system (ETI system), 126

Erosions, 48

ETI system. See Erosion translationinvariant system

Euclidean distance, 19

Euclidean domain, 59

Euclidean metric, classical, 33

Euclidean morphological operators. See Minkowski operators

Euclidean space, 60

Euclidean topology, 99

Everhart-Thornley detector, 227-228

\section{$\mathbf{F}$}

Fast Fourier transform (FFT), 334-335

FEG. See Field emission gun

FFT. See Fast Fourier transform

Field emission gun (FEG), 282-283

Finite impulse response filters (FIR filters), 156, 165-166

Finite-dimensional image spaces continuous domain, 66-67

discrete domain, 65-66
Finite-dimensional linear spaces, 57, 62, 68

Finite-dimensional nonlinear vector spaces, 119

Finite-dimensional spaces, 65

Finite-dimensional vector spaces, 62

linear operators on

matrix representation of, 74-75

spectral representation of, 76-78

weighted minimax spaces, 139-142

FIR filters. See Finite impulse response filters

Flat operators

grey-level median, 161

set generator, 161

signal operator, 159-160

stack filters, 161

Forming process, 265-266

French National Centre for Scientific

Research (CNR), 247-248

Function lattice, 84-85

pointwise partial ordering, 84

power lattice, $84-85$

signal lattices, 85

vector lattices, 85

Fuzzy dilation, 134-135

Fuzzy norms, 131-132

dual-translated dual impulses, 133-134

fuzzy convolutions, 133

fuzzy operations, 136, $137 \mathrm{f}$

image analysis, 138-139

intersection norm, 133

lattice image processing, 131-132

lattice-fuzzy openings and closings, 135-136

mathematical morphology, 132

morphological flat dilation and erosion, 136-138, $138 \mathrm{f}$

t-conorms, 132

$t$-norms, 132, 135

union norm, 133

G

Gold

nanoparticles, STEM micrographs, $291 \mathrm{f}$ nanostructure, HR-TEM simulations, $288 \mathrm{f}$

Gold-iridium alloy, 298-299

Gold-palladium nanoparticles, 304, 306-308 
core-shell structures, 312-314

energy dispersive analysis, 308

intercalation, $307 \mathrm{f}$

Gold-palladium-gold nanoparticles, 311-312, 316-318

orientation, 312

Granta Electronics, 274

Graphispot chart recorder, 264-265

Gravitational clustering, 25

adaptation

aggregation, 26-27

gravitational interaction, $25 \mathrm{f}$

grey-level image, 26

histogram, 26

to image processing, 25-26

LIP framework, 26

implementation

algorithm, 27

grey-level axis, $27 \mathrm{f}$

heart, 28-30

initialization, $27-28$

SM algorithm, 27

metrics selection

applications, 34

classical Euclidean metric, 33

logarithmic metric, 33

SM and OA algorithms, 33

notations, 25

principle, 25

results, 34, 35f, 37f

RGB components, 36-37

Grey-level area opening, 104-105, $105 f$

Grey-level images, 50-52

Grey-level median, 161

\section{$\mathrm{H}$}

HAADF detector. See High-angle annular dark-field detector

HAADF-STEM. See High-angle annular dark-field scanning transmission electron microscopy

HAC. See Hierarchical ascendant classification

Hamel basis, 62, 117

HCP structure. See Hexagonal close-packed structure

HDN. See Hydrodenitrogenation
HDO. See Hydrodeoxygenation

HDS. See Hydrodesulfurization

Heijman's monograph, 57

Hexagonal close-packed structure (HCP structure), 311-312

Hierarchical ascendant classification (HAC), 2-3

Benzécri algorithm, 17-18

Benzécri HAC, 14-15

hierarchy, 15-16

ultrametrics, 16-17

building steps

with Euclidean metric, 20f

with logarithmic metric, $20 \mathrm{f}$

classical quad tree decomposition, $24 \mathrm{f}$

color, 22f, $23 \mathrm{f}$

comparison, $23 \mathrm{f}$

dendrogram representation, $20 \mathrm{f}$

grey-level, 21f, $22 \mathrm{f}$

initial image values, $19 \mathrm{f}$

intermetric comparison, $23 \mathrm{f}$

random color LUT, 22f, $24 \mathrm{f}$

results and examples, 21-24

specific approach, 19-21

2D images, 17

High-angle annular dark-field detector (HAADF detector), 280-281, 285-286

High-angle annular dark-field scanning transmission electron microscopy (HAADF-STEM), 285-286

High resolution electron microscope (HREM), 272-273

High-resolution transmission electron microscopy (HR-TEM), 281

High-voltage electron microscope (HVEM), 245-246

High-voltage power transmission, 256-257

Hilbert space, 64, 73

Hit-miss transformation, 90, 155

Hölder inequality, 66

Homomorphisms, 88-89

HR-TEM. See High-resolution transmission electron microscopy

HREM. See High Resolution Electron Microscope 
HVEM. See High-voltage electron microscope

Hydrodenitrogenation (HDN), 319-320

Hydrodeoxygenation (HDO), 319-320

Hydrodesulfurization (HDS), 319-320

\section{I}

ILTI operator. See Increasing linear translation-invariant operator

Image algebra, 54-55

Image function model, 65

Image lattices

distributive lattices, 85-86

grey-level image signals, 86

1.s.c. functions, 86

set lattice, 85-86

shapes and images, 85

spatiotemporal image signals, 85

u.s.c. functions, 86

vector-valued images, 86

Image operators, 119

on CLW, 119

image signals, 119

matrix representations

basis vectors, 140

CWL, 139

dual vector representation, 140-141

finite-dimensional function lattice, 139

linear vector space, 141

min-`matrix product, $₫, 141$

pulse vectors, 140

V-translations, 139

semi-atoms, 119-120

V-translations, 120

Increasing linear translation-invariant

operator (ILTI operator), 166

Increasing operators, 88-89

automorphisms, 89

hit-miss transformation, 90

homomorphisms, 88-89

lattice-based image analysis, 89

structuring element, 90

Infimal convolution, 54

Infimum, 78

Infinite-dimensional image spaces

continuous-domain, 66-67

discrete-domain, 65-66
Infinite-dimensional linear spaces, 57, 62, 68

Injector system, 262

Invariance domain, 102

Isometry isomorphism, 71

Isomorphic linear spaces, 68

Isomorphism, 71. See also Dual automorphism

algebraic isomorphism, 71

isometry isomorphism, 71

topological isomorphism, 71

unitary operator, 71

Isotone. See Increasing operators

\section{J}

Janus structure, $305 \mathrm{f}$

Jet propulsion Laboratory, 275

Jolly Miller pub, 261-262

K

K-means algorithm. See also

Multithresholding; Region growing

method

comments, 6-7

Köhler's approach, 4

La Rochelle port, $6 \mathrm{f}$

Nuées dynamiques, 4

principle, 4-6

Kernel, adaptive, 97

Kernel elements

translation invariant operators, 146

Bas $(\psi), 157$

denoising, 157-158

dual set operator, 147

Hamel basis, 62, 158-159

increasing set operators, 146

kernel property, 146

minimal kernel elements, 147-148

minimal subcollection, 149

morphological basis, 159

non-flat, 159

nondegenerate operators, 146

set dilation, 146

set erosion operator, 146

set operator bases, 150

signal operator, 156-157

TI filters, 158

u.s.c. function, $148-149$ 
weighted erosions and dilations, 157

Kernel operators, 145-146

Kernel representation theory, 145, 156

Köhler's approach, 3-4

kth rank set operator, 152

closed formula, 153

dual set operator, 153

5-pixel discrete rhombus, 152-153, $153 \mathrm{f}$

\section{$\mathbf{L}$}

1.s.c. function. See Lower semi-continuous function

Lattice, 78-79

adjunctions, 57-58

atomic, 82

chain, 81

distributive, $79-80$

isomorphism, 88-89

linear space, 81

modular, 80

morphology, 52

operations, $79 \mathrm{t}$

operators, $57-58$

power set, 81

projections, 57-58

ring of sets, 81

Lattice automorphism.

See Lattice-isomorphism

Lattice spaces, 78

adjoint dilation, 92

adjoint erosion, 92

adjunction, 92

Boolean lattices, 83

convergence, 98

duality in lattices, 79

duality principle, 78

function lattices, 84

image lattices, 85

isomorphism, 79

monotone lattice operators, 88

order projections, 101

partial ordering, 78

properties, 79

semilattices, 80

shift-varying operators, 95

signal operators, 87

sublattices, 80 sup-generators, 82

Layered material electron microscopy, 319320

co-doped $\mathrm{MoS}_{2} / \mathrm{WS}_{2}$ nanowire catalysts, 326-327

BF-STEM images, 327f, $328 \mathrm{f}$

density functional theory calculations, 331-334

EDAX line scans, 330-331, 331f

HAADF-STEM images, 327f, $328 \mathrm{f}$

high-resolution, 329, 330f

low-magnification, 327-329, $329 \mathrm{f}$

perspective and top views, $332 \mathrm{f}$

SEM micrographs, $333 \mathrm{f}$

$\mathrm{MoS}_{2}$ nanoplates

FFT plates, 334-335

HR-TEM micrograph, 336-337, 337f

nanohexagonal plates, 334

superimposition, $336 \mathrm{f}$

ultra-high-resolution BF-STEM

image, 334, 335f

$\mathrm{MoS}_{2}$ nanowire catalysts, 320-321

HAADF-STEM image, 321, 322f

helical structure, 321

side and transversal views, $324 \mathrm{f}$

slab-size effects, 321-325

supported on $\mathrm{Al}_{2} \mathrm{O}_{3}, 323 \mathrm{f}$

zizzag-and armchair-terminated $\mathrm{MoS}_{2}$ sheets, 325-326

transition metal chalcogenides, 319-320

Left adjoint. See Adjoint erosion

Legendre-Fenchel conjugate transform, 54

Levelings

object-oriented filters, 109

self-dual reconstruction filter, 109-110

Linear convolution, 76

Linear isometry. See Isometry isomorphism

Linear operators

algebraic definitions, 68

isomorphism, 71, 79

normed spaces, 69

bounded, 69

continuous linear maps, 69

infinite linear superposition principle, 69

representation, 74

finite-dimensional vector spaces, 74 
Linear operators (Continued)

linear combination, 74

ring of linear operators, 75

Riesz representation theorem and linear convolution, 75

self-adjoint operator, 70

spectral representation, 76

Linear projections

algebraic decomposition, 72

algebraic complement, 72

linear space, 72

orthogonal projections, 72-73

least square approximation, $73-74$

orthogonal complement, 72-73

properties, 73

Linear shift-invariant filter (LSI filter), 165

Linear signal processing, 57

Linear spaces, 81

algebraic structure, 61

conditions, 61-62

finite and infinite dimensional, 62

linear combination of points, 62

linear subspace, 62

linearly independent, 62

theorem, 62

continuous functions, 67

finite-dimensional spaces, 65

geometric structure

inner product linear spaces, 64

orthogonal, 64

properties, 64

Pythagorean theorem, 64

infinite-dimensional image spaces

continuous-domain, 66-67

discrete-domain, 65-66

linear operators

algebraic definitions, 68

isomorphisms, 71

normed spaces, 69

self-adjoint operator, 70

linear projections

algebraic decomposition, 72

orthogonal projections, 72-74

topological structure

Cauchy test, 63-64

convergent sequence, 63

equivalent metrics, 63 metric space, 63

norm, 63

viewpoints, 61

LIP model. See Logarithmic image processing model

Lipschitz continuous functions, 67, 87

Logarithmic image processing model (LIP model), 3, 7

digitized images, 2

grey-scale digitization, 2

HAC, 2-3

homogeneity or texture, 2

image segmentation, 2

reflections

grey-level images, 40

optical vector space, 41

using remarks, 42

transmittance law, 40

transmittances formula, 41

Logarithmic metrics, 33

Lower semi-continuous function (1.s.c. function), 86

LSI filter. See Linear shift-invariant filter

M

Matrix rank, 143

Max- ${ }^{\star}$ dependent combination, 117

Max- ${ }^{\star}$ independent combination, 117

Max-plus algebra, 55. See also Minimax algebra; Nonlinear matrix algebra

Max-plus convolution, 54

Max-plus image operators, 129

kernel, 130

max-plus case, 130

shift invariance, 130

Max-plus matrix algebra, 142

conjugate matrix, 142

definite and metric matrix, 144

eigenvalue, 144

eigenvectors, 144-145

graph of matrix, 143-144

matrix rank, 143

optimization problem, 142-143

Max-product image operators, 130-131

isomorphism, 131

sup-product convolution, 130-131

Mean squared error (MSE), 73-74 
Median, 152

adaptive, 170-171

Metal catalysts

on $\gamma-\mathrm{Al}_{2} \mathrm{O}_{2}, 301-304$

metal- $\mathrm{TiO}_{2}, 304$

$\mathrm{Pt} / \gamma-\mathrm{Al}_{2} \mathrm{O}_{3}$ catalyst, $303 \mathrm{f}$

on crystalline substrate, 294-295

$\mathrm{Au} / \mathrm{Ir}$ alloy support in $\mathrm{TiO}_{2}, 298 \mathrm{f}$

$\mathrm{Au} / \mathrm{TiO}_{2}$ catalyst image, $297 \mathrm{f}$

EELS analysis, 298-299

signal-to-noise ratio, 296-298

STEM-HAADF image, $296 \mathrm{f}$

on noncrystalline substrate, 299

aberration corrected TEM, $302 \mathrm{f}$

CNO, 299

DF and BF, 301f

iridium support on Vulcan carbon, $300 f$

Z-contrast images, 300-301

Metric matrix, 144

Minimal kernel elements, 147-148

Minimax algebra, 55, 110

clodum, 110

lattice-ordered monoids, 111

lattice-ordered group, 111

lattice-ordered monoid, 111-112

poset, 111

semilattice-ordered monoid, 111

max-plus mathematical morphology, 110

nonlinear space, 110

Minkowski operators, 50-52, 90

Minkowski set dilation, 93

Minkowski set erosion, 94

Minkowski weighted erosion, 95

Modular lattice, 80

Monotone lattice operators

decreasing operators

complete lattices, 91

dual homomorphisms, 90

dual isomorphism, 91

grey-level images, 91

signal operators, 91

thresholding operators, 91

increasing operators, 88-89

automorphisms, 89

hit-miss transformation, 90

homomorphisms, 88-89 lattice-based image analysis, 89

structuring element, 90

lattice structures, 88

Morphological filters, 101

Morphological operators

developments, 50-52

algebraic structure, 52-53

classic mathematical morphology, 50-52

clodum, 56-57

convex analysis and optimization, 54

finite-dimensional case, 55-56

image algebra, 54-55

image analysis applications, 52

lattice framework, 53

lattice operators, 52

minimax algebra, 55

nonlinear dynamical systems, 53-54

linear spaces, 61

minimax algebra, 110

notation, 60

representation theory, 47

Morphological set operators, 150

constraints, 166

discrete linear filter, 167

erosions supremum, 166-167

$k$ th rank set operator, 152

closed formula, 153

dual set operator, 153

5-pixel discrete rhombus, 152-153, $153 \mathrm{f}$

median, 152

Minkowski closing, 150-151

Minkowski dilation, 150

Minkowski erosion, 150

Minkowski opening, 150-151

moving average filter, 167

opening and closing basis sets, 151, $152 \mathrm{f}$

upper support, 166

window transformations, 153

Morphology, adaptive, 97-98

Morrison air-raid shelter, 191-192

$\mathrm{MoS}_{2}$ catalysts, 319-320

nanoplates

FFT plates, 334-335

HR-TEM micrograph, 336-337, $337 f$

nanohexagonal plates, 334 
$\mathrm{MoS}_{2}$ catalysts (Continued)

superimposition, $336 \mathrm{f}$

ultra-high-resolution BF-STEM

image, 334, 335f

nanowire catalysts, 320-321

armchair-terminated $\mathrm{MoS}_{2}$ sheets, 325-326

HAADF-STEM image, 321, 322f

helical structure, 321

side and transversal views, $324 \mathrm{f}$

slab-size effects, 321-325

supported on $\mathrm{Al}_{2} \mathrm{O}_{3}, 323 \mathrm{f}$

zizzag- terminated $\mathrm{MoS}_{2}$ sheets, 325-326

MSE. See Mean squared error

Multimetallic systems, 318-319

Multithresholding, 3. See also K-means algorithm

contrast curve, 4

Köhler's approach, 3-4, $5 \mathrm{f}$

techniques, 3

\section{N}

Nanobeam electron diffraction (NBD),

$$
\text { 311-312 }
$$

Nanoribbons, 280

National Autonomous University of

$$
\text { Mexico (UNAM), 294-295 }
$$

NBD. See Nanobeam electron diffraction

Neighborhoods, adaptive, 97-98

Nonlinear functional analysis, 50

Nonlinear matrix algebra, 55

Nonlinear spaces

algebraic structure, 114

clodum, 114

CWL space, 116

WL, 114-115

complete weighted lattices functions

CWL space, 118

pointwise functions, 118

self-conjugate element, 119

sup- ${ }^{\star}$ combination, 116-117

Hamel basis, 117

max- ${ }^{\star}$ and min- ${ }^{\star \prime}$ combination, 117

Normed spaces, 69

bounded, 69

continuous linear maps, 69 infinite linear superposition, 69

Notation

image domain, 60

image operators, 60-61

linear operators, 60

morphological operators, 60

Null element, 78-79

Null space, 68

\section{0}

Optimized automated algorithm (OA algorithm), 31, 36f. See also

Sampling of mechanical model algorithm (SM algorithm)

acceptable solutions, 32

amalgamation, 31-32

flowchart, $32 \mathrm{f}$

initialization, 31

moving bodies, 32-33

Order continuity, 100

Order projections, 101

alternating sequential filters, 108-109

lattice theoretic properties, 101

extensive and anti-extensive operators, 102

openings and closings, 102-103

levelings, 109

Orthogonal projections, 72-73

least squares approximation, 73-74

orthogonal complement, 72-73

properties, 73

Ostwald ripening effects, 316-318

$\mathbf{P}$

Palladium-gold-palladium nanoparticles, 311-312

Partial differential equation (PDE), 53-54

Partial ordering, 78

Parts hierarchy, 15

PDE. See Partial differential equation

Peppers image, 21

Planar set operators, 170

Polyvinylpyrrolidone (PVP), 304

POS. See Product-of-sum

Power lattice. See Function lattice

Power set, 81

PPRIC. See Pulp and Paper Research Institute of Canada 
Prime implicant, 161-162

Product-of-sum (POS), 161-162

Pseudonorm. See Seminorm

Pulp and Paper Research Institute of Canada (PPRIC), 222

PVP. See Polyvinylpyrrolidone

\section{Q}

Quantization error effects, 99

$\mathbf{R}$

Radial openings, 103-104, 104f

Real linear space, 62

Reconstruction closing

grey-level reconstruction filterings, $108 \mathrm{f}$

image simplification, 107-108

opening, 106

conditional dilation, 106, $107 \mathrm{f}$

grey-level reconstruction opening, 107

marker, 107

Red-green-blue image (RGB image), 6-7

Reflection microscope, 193

Region growing method. See also K-means algorithm

classical

aggregation process, 8

equivalence relation, 8

grey-level distribution, 11

using pixels, 8

using remarks, 9

single linkage effects, 9, 11f

3D image segmentation, 9-11

transitivity saturation, 9

LIP version

using commands, 12

efficiency, 11

using formula, 11-12

logarithmic single linkage, $12 \mathrm{f}$

objectives, 13

$3 \mathrm{D}$ region growing segmentation, $12 \mathrm{f}$

notations

grey-level image, 7

LIP model, 7 multiplication calculation, 8

space of images, 7-8

systolic approach, $14 \mathrm{f}$

classical version, 13

using remarks, 13-14

Revol's approach, 13

seed's exclusion, 14

Representation theory, 47

computational theory, 49

digital image processing, 49

dilations and erosions, 48

finite-dimensional case, 58-59

image operators, $47-48$

image representations, 47

image-processing tasks, 50

lattice adjunctions, 57-58

lattice structure, 58

linear and morphological image simplification, $51 \mathrm{f}$

linear signal processing, 57

minimal kernel elements collection, 59

Minkowski openings, 60

morphological filterings, 50

morphological operators, 48-49

theory, 57

nonlinear functional analysis, 50

non-monotone TI operator, 59

signal algebra, 58

Research Department, 192

Revol's approach, 13

RGB image. See Red-green-blue image

RHS. See Right-hand side

Riesz's representation theorem, 57

discrete-time linear convolution, 76

impulse response, 76

linear convolution, 76

linear transformation, 75

time signals, 75

Right adjoint. See Adjoint dilation

Ring of sets, 81

Rolls-Royce Merlin engines, 192

Ronchigram, 293

calculated, 294f

for different focus conditions, $295 \mathrm{f}$

experimental, $294 \mathrm{f}$

focus spot, 293 


\section{$\mathrm{S}$}

Sailing, 223-224

Sampling of mechanical model algorithm

(SM algorithm), 27, 34-36, 34f.

See also Optimized automated

algorithm (OA algorithm)

flow chart, 27, 29f

gravitational clustering, $27,39 \mathrm{f}$

initialization

integer positions, 27

optimal number search, $32 \mathrm{f}$

merging trees, $38 \mathrm{f}, 39 \mathrm{f}$

steps, $28-30$

using cases, 30-31

using loop equations, 30

movement quantity normalization, 29-30

velocities and positions, 30

Sanatron, 204

Sash window, 254-255

Scale parameter, $51 \mathrm{f}$

Scanning electron microscope (SEM), 193-194. See also Transmission electron microscope (TEM)

detector system, 197

electron optical projects, 200-201

electron spot, 196

electron trajectory tracer, 203

Ellis Cosslett's electron microscopy, $202 \mathrm{f}$

imaging, 280-281

improvement in efficiency, 199

limitation, 194-195

magnification, 196

McMullan's original incarnation, $198 \mathrm{f}$

practical electronics work, 198

principles, 195-196

proceedings, 202

schematic diagram, $195 \mathrm{f}$

Sheila Smith at controls, 201f

technical problems, 199

transmission microscope, 193

visual acuity, 202

Scanning transmission electron microscopy (STEM), 221, 280-281

aberrations, 287

correction, 288

CTF, 287 gold particles, 290, 291f

HR-TEM simulations, $288 \mathrm{f}$

using image metal nanoparticles, 290

intensity profiles, $290 \mathrm{f}$

Scherzer defocus, 287-288

simulation TEM micrographs

calculation, $289 \mathrm{f}$

Z-contrast technique, 288-290

bimetallic gold-palladium nanoparticles, $281 \mathrm{f}$

EDS spectrum detector, 286f

HAADF-STEM, 285-286

information transfer, in phase-contrast imaging, 281

operational modes, 281, 282f

optical arrangement, 282

practical guide, 291

electron beam sketches, 291-292, $292 \mathrm{f}$

electron beam trajectories, $293 \mathrm{f}$

focus conditions, $295 \mathrm{f}$

in-focus and underfocus, 293

resolution, 291

ronchigram, 293, 294f

spot size and aperture, 292-293

principles

BF images, 283

BF-CTEM, 283

FEG, 282-283

Fourier transform, 285

high-resolution electron microscopy, 284

operation modes, 283

relativistic Dirac equation, 284-285

Scherzer condition, 287

Scientific Digital Imaging plc (SDI), 275

Self-adjoint operator, 70

examples, $70-71$

properties, 70

Self-compositions, 88

SEM. See Scanning electron microscope;

Structuring element map

Semi-atom, 82

Semi-graphitic carbon, 280

Semilattices, 80

Seminorm, 63

Set generator, 159-160 
Shift-varying operators, 95-97

adaptive morphology, 97-98

adaptive neighborhoods, 97-98

amoeba filters, 97

geometry adaptive filters, 97

SV operators, kernel and basis representation for, 98

SEM, 95-96

adjoint erosion, 96

grey-level image functions, 96

Shockley partial dislocation (SPD),

$$
\text { 312-314 }
$$

Signal operators, 87

SM algorithm. See Sampling of mechanical model algorithm

SMI. See Strongly max-plus independent

Smith, Sheila Vernon, 201

at Cambridge, 205-206

Coach's prow, $207 \mathrm{f}$

Fairwell to crew, 206-207

motorcycling, $205 \mathrm{f}$

time for music, 208-209

SOP. See Sum-of-products

Spatially varying operators (SV operators), 95

adaptive median, 170-171

erosions, 168

image operators, 168

increasing operator, 169

planar set operators, 170

polar coordinates, 170

representations, 168

SEM, 168-170

SV kernel, 169

Spatially varying operators. See Shift-varying operators

SPD. See Shockley partial dislocation

Spectral representation

eigenvalues, 77

finite-dimensional vector spaces on, 76

resolution of the identity, 77

Spruce wood fiber, $222 \mathrm{f}$

Stack filters

basis representation, 164-165

grey-level output signal, 164

Standards Room, 192

State-of-the-art simulations, 321-325
STEM. See Scanning transmission electron microscopy

Stratified hierarchy, 16

Strongly max-plus independent (SMI), 143

Structuring element, 90

Structuring element map (SEM), 95-96

adjoint erosion, 96

grey-level image functions, 96

adaptive kernel, 97

adaptive window, 96

Structuring function. See also Structuring element map (SEM)

Subbasis, 177-178

Sublattice, 80

sup-closed lattice, $80-81$

underlattice, 80

Sum-of-products (SOP), 161-162

Sup- ${ }^{\star}$ combination, $116-117$

Hamel basis, 117

max-` and min- ${ }^{\star}{ }^{\prime}$ combination, 117

Sup-closed lattice, $80-81$

Sup-generators, 82

atom, 82

dual atom, 82

dual semi-atoms, 82

semi-atom, 82

Sup-semilattice, 80

Suphomomorphism, 88-89

Supremal convolution, 54, 95

Supremum norm, 66-67, 78

SV operators. See Spatially varying operators

Systolic approach, $14 \mathrm{f}$

classical version, 13

using remarks, 13-14

Revol's approach, 13

seed's exclusion, 14

\section{T}

TEM. See Transmission electron microscope Testing motorcycles, 190

Thorne, Ken, 208

Three-dimensional image segmentation (3D image segmentation), 9-11

Three-layer nanoparticle, 309, $310 \mathrm{f}$

TI. See Translation-invariant

Tiplady's, 245

Topological isomorphism, 71 
Transition metal chalcogenides, 319-320

Translation-invariant (TI), 50-52

Bas $(\psi), 157$

denoising, 157-158

dual set operator, 147

dual subbasis, 178

flat area opening, 179, $179 \mathrm{f}$

Hamel basis, 158-159

increasing operators

adjoint erosions, 176

adjunction, 175

CWL, 174-175

dual kernel, 176

kernel, 175

semi-atom, 175

T-invariant dilation, 176

increasing set operators, 146

kernel property, 146

minimal kernel elements, 147-148

minimal subcollection, 149

morphological basis, 159

non-flat, 159

non-increasing operators

closed interval, 171

hit-miss operations, 173-174

inf-generating operator, 172

inf-separable, 173

kernel maximal closed intervals, 174

sup-generating operator, 171-172

sup-separable, 173

TI set operator, 172

nondegenerate operators, 146

openings and closings, 177

set dilation, 146

set erosion operator, 146

set operator bases, 150

signal operator, 156-157

subbasis, $177-178$

TI filters, 158

u.s.c., 148-149

weighted erosions and dilations, 157

Translations

CWL signal space, 126

horizontal and vertical translations, 125

impulse, 125-126

monoid of signal erosions, 125

polar motions, 124
Transmission electron microscope (TEM), 193-194. See also Scanning electron microscope (SEM)

aberrations, 287

correction, 288

CTF, 287

gold particles, 290, 291f

HR-TEM simulations, $288 \mathrm{f}$

using image metal nanoparticles, 290

intensity profiles, $290 \mathrm{f}$

Scherzer defocus, 287-288

simulation TEM micrographs

calculation, $289 \mathrm{f}$

Z-contrast technique, 288-290

bimetallic gold-palladium nanoparticles, $281 \mathrm{f}$

EDS spectrum detector, $286 f$

HAADF-STEM, 285-286

imaging, 280-281

information transfer in phase-contrast imaging, 281

operational modes, 281, $282 \mathrm{f}$

optical arrangement, 282

practical guide, 291

electron beam sketches, 291-292, $292 \mathrm{f}$

electron beam trajectories, $293 \mathrm{f}$

focus conditions, $295 \mathrm{f}$

in-focus and underfocus, 293

resolution, 291

ronchigram, 293, 294f

spot size and aperture, 292-293

principles

BF images, 283

BF-CTEM, 283

FEG, 282-283

Fourier transform, 285

high-resolution electron microscopy, 284

operation modes, 283

relativistic Dirac equation, 284-285

Transmittance law, 40

Triumph Twin Motorbike, 205

Two-dimensional algorithm (2D

algorithm), 15. See also Benzécri

algorithm

aggregation index, 18 
image grey scale, 18

pseudo-code, $18 \mathrm{f}$

time-consuming part, 18

Two-dimensional Gaussian function (2D

Gaussian function), 50

\section{U}

u.s.c. function. See Upper semi-continuous function

Ultrametrics, 16-17

Umbra function, 54

UNAM. See National Autonomous University of Mexico

Unconditional convergence, 63-64

Underlattice, 80

Uniform convergence, 66-67

Unitary operator, 71

Upper semi-continuous function (u.s.c. function), 86

\section{V}

Vectors. See also Linear spaces

Vertical translations (V-translations), 119120

function dilations, $120-121$

impulse response map, 122-123

invariant function, 121

linear superposition, 122

nonlinear superposition principle, 122

scalar erosions, 122

\section{W}

WBDF technique. See Weak-beam darkfield technique
Weak-beam dark-field technique

(WBDF technique), 311-312

Weighted lattice (WL), 114-115

Window, adaptive, 96

Window transformations, 153

application area, 156

comparison, 154-155

discrete set operator, 153

finite union of erosions, 155

grey-level image processing, 155-156

hit-miss transformation, 155

increasing window operator, 155

locally defined operator, 154

matched filter, 154

one-to-one correspondence, 154

shape collection, 153-154

WL. See Weighted lattice

$\mathrm{WS}_{2}$ catalyst, 319-320

nanowire catalysts, 326-334

\section{$X$}

X-ray detector, 285-286

X-ray photoelectron spectroscopy, 326-327

Y

Yarmouth, 205

Yorkshire, 217-219

\section{Z}

Z-contrast technique, 288-290

Zigzag nanoribbons, 321-325 
This page intentionally left blank 


\section{Contents of Volumes 151-176}

\section{Volume $151^{1}$}

Claas Bontus and Thomas Köhler, Reconstruction algorithms for computed tomography

Laurent Busin, Nicolas Vandenbroucke and Ludovic Macaire, Color spaces and image segmentation

Glenn R. Easley and Flavia Colonna, Generalized discrete Radon transforms and applications to image processing

Tomáš Radlička, Lie agebraic methods in charged particle optics

Valerie Randle, Recent developments in electron backscatter diffraction

\section{Volume 152}

Nina S.T. Hirata, Stack filters: from definition to design algorithms

Sameen Ahmed Khan, The Foldy-Wouthuysen transformation technique in optics

Saverio Morfu, Patrick Marquié, Brice Nofiélé and Dominique Ginhac, Nonlinear systems for image processing

Tohru Nitta, Complex-valued neural network and complex-valued backpropagation learning algorithm

Jérôme Bobin, Jean-Luc Starck, Y. Moudden and M.J. Fadili, Blind source separation: the sparsity revoloution

Ray L. Withers, "Disorder": structured diffuse scattering and local crystal chemistry

\section{Volume 153}

Aberration-corrected Electron Microscopy Harald Rose, History of direct aberration correction

Maximilian Haider, Heiko Müller and Stephan Uhlemann, Present and future hexapole aberration correctors for high-resolution electron microscopy
Ondrej L. Krivanek, Niklas Dellby, Robert J. Kyse, Matthew F. Murfitt, Christopher S. Own and Zoltan S. Szilagyi, Advances in aberration-corrected scanning transmission electron microscopy and electron energyloss spectroscopy

Philip E. Batson, First results using the Nion third-order scanning transmission electron microscope corrector

Andrew L. Bleloch, Scanning transmission electron microscopy and electron energy loss spectroscopy: mapping materials atom by atom

Florent Houdellier, Martin Hÿtch, Florian Hüe and Etienne Snoeck, Aberration correction with the SACTEM-Toulouse: from imaging to diffraction

Bernd Kabius and Harald Rose, Novel aberration correction concepts

Angus I. Kirkland, Peter D Nellist, Lan-yun Chang and Sarah J. Haigh, Aberrationcorrected imaging in conventional transmission electron microscopy and scanning transmission electron microscopy

Stephen J Pennycook, M.F. Chisholm, A.R. Lupini, M. Varela, K. van Benthem, A.Y. Borisevich, M.P. Oxley, W. Luo and S.T. Pantelides, Materials applications of aberration-corrected scanning transmission electron microscopy

Nobuo Tanaka, Spherical aberration-corrected transmission electron microscopy for nanomaterials

Knut Urban, Lothar Houben, Chun-lin Jia, Markus Lentzen, Shao-bo Mi, Andreas Thust and Karsten Tillmann, Atomic-resolution aberration-corrected transmission electron microscopy

Yimei Zhu and Joe Wall, Aberration-corrected electron microscopes at Brookhaven National Laboratory

1 Lists of the contents of volumes 100-149 are to be found in volume 150; the entire series can be searched on ScienceDirect.com 


\section{Volume 154}

Henning F. Harmuth and Beate Meffert, Dirac's Difference Equation and the Physics of Finite Differences

\section{Volume 155}

Dmitry Greenfield and Mikhail Monastyrskiy, Selected Problems of Computational Charged Particle Optics

\section{Volume 156}

Vasileios Argyriou and Maria Petrou, Photometric stereo: an overview

Fred Brackx, Nele de Schepper and Frank Sommen, The Fourier transform in Clifford analysis

Niels de Jonge, Carbon nanotube electron sources for electron microscopes

Erasmo Recami and Michel Zamboni-Rached, Localized waves: a review

\section{Volume 157}

Mikhail I. Yavor, Optics of charged particle analyzers

\section{Volume 158}

Péter Dombi, Surface plasmon-enhanced photoemission and electron acceleration with ultrashort laser pulses

Brian J. Ford, Did physics matter to the pioneers of microscopy?

Jérôme Gilles, Image decomposition: theory, numerical schemes, and performance evaluation

Stina Svensson, The reverse fuzzy distance transform and its use when studying the shape of macromolecules from cryo-electron tomographic data

Marc van Droogenbroeck, Anchors of morphological operators and algebraic openings

Dong Yang, Shiva Kumar and Hao Wang, Temporal filtering technique using time lenses for optical transmission systems

\section{Volume 159}

Cold Field Emission and the Scanning Transmission Electron Microscope

Albert Victor Crewe, The work of Albert Victor Crewe on the scanning transmission electron microscope and related topics

Lyn W. Swanson and Gregory A. Schwind, A review of the cold-field electron cathode

Joseph S. Wall, Martha N. Simon and JamesF. Hainfeld, History of the STEM at Brookhaven National Laboratory

Hiromi Inada, Hiroshi Kakibayashi, Shigeto Isakozawa, Takahito Hashimoto, Toshie Yaguchi and Kuniyasu Nakamura, Hitachi's development of cold-field emission scanning transmission electron microscopes

Peter W. Hawkes, Two commercial STEMs: the Siemens ST100F and the AEI STEM-1

Ian R.M. Wardell and Peter E. Bovey, A history of Vacuum Generators' 100-kV STEM

H. Sebastian von Harrach, Development of the 300-kV Vacuum Generators STEM (1985-1996)

Bernard Jouffrey, On the high-voltage STEM project in Toulouse (MEBATH)

Andreas Engel, Scanning transmission electron microscopy: biological applications

Kenneth C.A. Smith, STEM at Cambridge University: reminiscences and reflections from the 1950s and 1960s

\section{Volume 160}

Zofia Baranczuk, Joachim Giesen, Klaus Simon and Peter Zolliker, Gamut mapping

Adrian N. Evans, Color area morphology scalespaces

Ye Pu, Chia-lung Hsieh, Rachel Grange and Demetri Psaltis, Harmonic holography

Gerhard X. Ritter and Gonzalo Urcid, Lattice algebra approach to endmember determination in hyperspectral imagery

Reinhold Rüdenberg, Origin and background of the invention of the electron microscope

H. Gunther Rudenberg and Paul G. Rudenberg, Origin and background of the invention of the electron microscope: commentary and expanded notes on Memoir of Reinhold Rüdenberg 


\section{Volume 161}

Marian Mankos, Vassil Spasov and Eric Munro, Principles of dual-beam low-energy electron microscopy

Jorge D. Mendiola-Santibañez, Iván R. TerolVillalobos and Israel M. Santillán-Méndez, Determination of adequate parameters for connected morphological contrast mappings through morphological contrast measures Ignacio Moreno and Carlos Ferreira, Fractional Fourier transforms and geometrical optics Vladan Velisavlevic, Martin Vetterli, Baltasar Berufell-Lozano and Pier Luigi Dragotti, Sparse image representation by directionlets Michael H.F. Wilkinson and Georgios K. Ouzounis, Advances in connectivity and connected attribute filters

\section{Volume 162}

Kiyotaka Asakura, Hironobu Niimi and Makoto Kato, Energy-filtered x-ray photoemission electron microscopy (EXPEEM)

Eireann C. Cosgriff, Peter D. Nellist, Adrian J. d'Alfonso, Scott D. Findlay, Gavin Behan, Peng Wang, Leslie J. Allen and Angus I. Kirkland, Image contrast in aberrationcorrected scanning confocal electron microscopy

Christopher J. Edgcombe, New dimensions for field emission: effects of structure in the emitting surface

Archontis Giannakidis and Maria Petrou, Conductivity imaging and generalised Radon transform: a review

Olivier Losson, Ludovic Macaire and Yanqin Yang, Comparison of color demosaicing methods

\section{Volume 163}

Wolfgang S. Bacsa, Optical interference near surfaces and its application in subwavelength microscopy

Ruy H.A. Farias and Erasmo Recami, Introduction of a quantum of time ("chronon"), and its consequences for the electron in quantum and classical physics

Andrew Neice, Methods and limitations of subwavelength imaging
A.Sever Škapin and P. Ropret, Identification of historical pigments in wall layers by combination of optical and scanning electron microscopy coupled with energy-dispersive spectroscopy

Markus E. Testorf and Michael A. Fiddy, Superresolution imaging-revisited

\section{Volume 164}

Amos Bardea and Ron Naaman, Magnetolithography: from the bottom-up route to high throughput

Román Castañeda, The optics of spatial coherence wavelets

Junchang Li, Yanmei Wu and Yan Li, Common diffraction integral calculation based on a fast Fourier transform algorithm

Marcel Teschke and Stefan Sinzinger, A generalized approach to describe the interference contrast and phase contrast method

Dokkyu Yi and Booyong Choi, Nonlinear partial differential equations for noise problems

Henning F. Harmuth, Harmuth corrigenda

\section{Volume 165}

Natalie Baddour, Two-dimensional Fourier transforms in polar coordinates

Neil V. Budko, Superluminal, subluminal, and negative velocities in free-space electromagnetic propagation

Rowan Leary and Rik Brydson, Chromatic aberration correction: the next step in electron microscopy

Michele Marrocco, Methods for vectorial analysis and imaging in high-resolution laser microscopy

Tomoya Sakai, Masaki Narita, Takuto Komazaki, Haruhiko Nishiguchi and Atsushi Imiya, Image hierarchy in Gaussian scale space

Yusuf Ziya Umul, The theory of the boundary diffraction wave

Emil Wolf, History and solution of the phase problem in the theory of structure determination of crystals from $x$-ray diffraction measurements

\section{Volume 166}

Valeriy Syrovoy, Theory of Intense Beams of Charged Particles 


\section{Volume 167}

Emmanuel de Chambost, A history of Cameca (1954-2009)

Johan Debayle and Jean-Charles Pinoli, Theory and applications of general adaptive neighborhood image processing

Mohamed ben Haj Rhouma, Mohamed Ali Khabou and Lotfi Hermi, Shape recognition based on eigenvalues of the Laplacian

Nicolas Loménie and Georges Stamon, Point set analysis

Leonid P. Yaroslavsky, Image recovery from sparse samples, discrete sampling theorem, and sharply bounded band-limited discrete signals

\section{Volume 168}

Luca Geretti and Antonio Abramo, The synthesis of a stochastic artificial neural network application using a genetic algorithm approach

Michel Jourlin, Josselin Breugnot, Frédéric Itthirad, Mohammed Bouabdellah and Brigitte Closs, Logarithmic image processing for color images

Rainer A. Leitgeb, Current technologies for high-speed and functional imaging with optical coherence tomography

Sergej A. Nepijko and Gerd Schönhense, Analysis of optical systems, contrast depth, and measurement of electric and magnetic field distribution on the object's surface in mirror electron microscopy

Chad M. Parish, Multivariate statistics applications in scanning transmission electron microscopy

Hidetaka Sawada, Fumio Hosokawa, Takeo Sasaki, Toshikatsu Kaneyama, Yukihito Kondo and Kazutomo Suenaga, Aberration correctors developed under the Triple $C$ project

Tobias Schulz, Martin Albrecht and Klaus Irmscher, Spatially resolved thermoluminescence in a scanning electron microscope

\section{Volume 169}

Erchan Aptoula and Sébastien Lefèvre, Morphological texture description of grayscale and color images
Vera Guarrera and Herwig Ott, Electron microscopy of ultracold gases

Konstantinos Konstantinidis, loannis Andreadis and Georgios Ch. Sirakoulis, Application of artificial intelligence to content-based image retrieval

Xingwei Yang, Daniel B. Szyld and Longin Jan Latecki, Diffusion on a tensor product graph for semi-supervised learning and interactive image segmentation

S.A. Nepijko and G. Schönhense, Electron holography for electric and magnetic field measurement and its application for nanophysics

\section{Volume 170}

A.S. Eggeman and P.A. Midgley, Precession electron diffraction

Ray Hill, John A. Notte and Larry Scipione, Scanning helium ion microscopy

Hone-Ene Hwang and Pin Han, Signal reconstruction algorithm based on a single intensity in the Fresnel domain

Kazuhisa Sato, Toyohiko J. Konno and Yoshihiko Hirotsu, Electron microscpy studies on magnetic $\mathrm{L} 10 \mathrm{FePd}$ nanoparticles

D.A. Zanin, H. Cabrera, L. de Pietro, M. Pikulski, M. Goldmann, U. Ramsperger, D. Pescia and John P. Xanthakis, Fundamental aspects of near-field emission scanning electron microcopy

\section{Volume 171}

Gregor Esser, Wolfgang Becken, Werner Müller, Peter Baumbach, Josep Arasa and Dietmar Uttenweiler, Derivation of the reflection equations for higher order aberrations of local wavefronts by oblique incidence

Lila Iznita Izhar and Maria Petrou, Thermal imaging in medicine

Jean-Michel Tualle, Derivation of the radiative transfer equation in a medium with a spatially varying refractive index: a review

Kamlesh Shrivas and Mitsutoshi Setou, Imaging mass spectrometry. Sample preparation, instrumentation and applications

Robert T. Thompson and Steven A. Cummer, Transformation optics 
Tobias Klein, Egbert Buhr and Carl Georg Frase, TSEM - a review of scanning electron microscopy in transmission mode and its applications

Michel Jourlin, Maxime Carré, Josselin Breugnot and Mohamed Bouabdellah, Logarithmic image procesing: additive contrast, multiplicative contrast and associated metrics

\section{Volume 172}

Jay Theodore Cremer, Neutron and x-ray microscopy, Part 1

\section{Volume 173}

Jay Theodore Cremer, Neutron and x-ray microscopy, Part 2

\section{Volume 174}

Silicon-based Millimeter-wave Technology. Measurement, Modeling and Applications

M. Jamal Deen and Ognian Marinov, Measurement techniques and issues

Guennadi A. Kouzaev, M. Jamal Deen and Natalia K. Nikolova, Transmission lines and passive components

Mohamed H. Bakr and Mohamed H. Negm, Modeling and design of high-frequency structures using artificial neural networks and space mapping

Oana Moldovan, Antonio Lázaro, François Danneville, Rodrigo Picos, Bogdan Nae, Benjamin Iniguez and M. Jamal Deen, Nanoscale FETs

M. Daneshmand and R.R. Mansour, RF MEMS switches and switch matrices
Natalia K. Nikolova, Maryam Ravan and Reza K. Amineh, Substrate-integrated antennas on silicon

\section{Volume 175}

Jay Theodore Cremer, Small Angle Scatter with Correlation, Scatter and Intermediate Functions

Jay Theodore Cremer, Nuclear Scatter of Neutron Spin States

Christian Dwyer, Atomic-Resolution Core-Level Spectroscopy in the Scanning Transmission Electron Microscope

Partha Pratim Mondal and Alberto Diaspro, Point Spread Function Engineering for Super Resolution Single- Photon and Multi Photon Fluorescence Microscopy

P. Murray and S. Marshall, A Review of Recent Advances in the Hit-or-Miss Transform

Stephen J. Sangwine, Perspectives on Colour Images Processing by Linear Vector Methods using Projective Geometric Transformations

\section{Volume 176}

Katsushige Tsuno, Damaschin loanoviciu, Early History of Wien Filters

Damaschin loanoviciu, Katsushige Tsuno, Aberration Theory of the Wien Filter

Katsushige Tsuno, Damaschin loanoviciu, Wien Filter Instrumentation

Katsushige Tsuno, Damaschin loanoviciu, Simulation of Multipole Wien Filters

Damaschin loanoviciu, Katsushige Tsuno, Wien Filter Applications to lons

Katsushige Tsuno, Damaschin loanoviciu, Application of Wien Filters to Electrons 
This page intentionally left blank 


\section{COLOR PLATES}

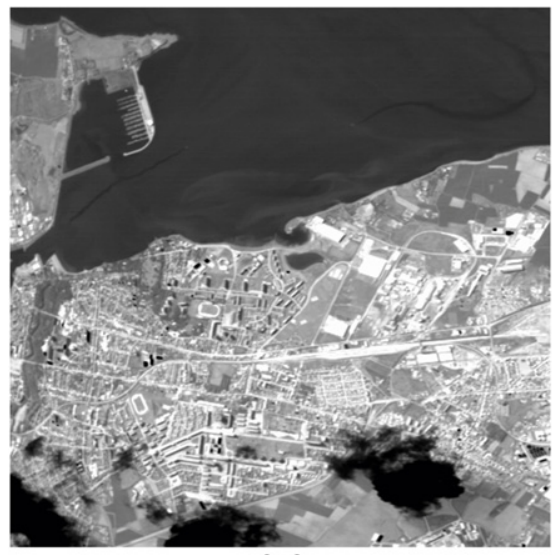

(a)

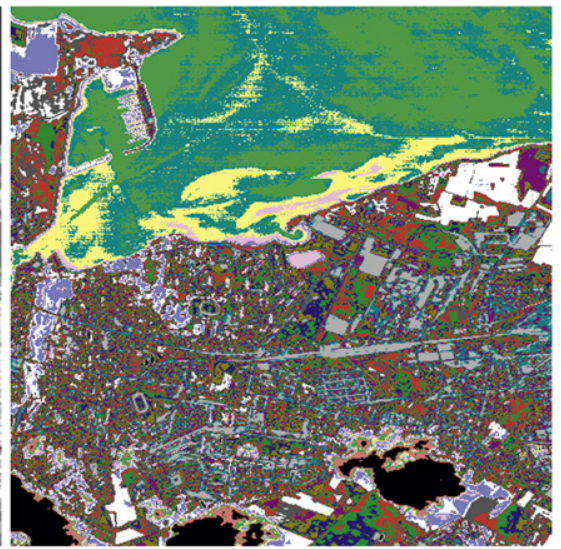

(b)

PLATE $2 b$ (Figure $2 b$ on page 6 of this volume) 

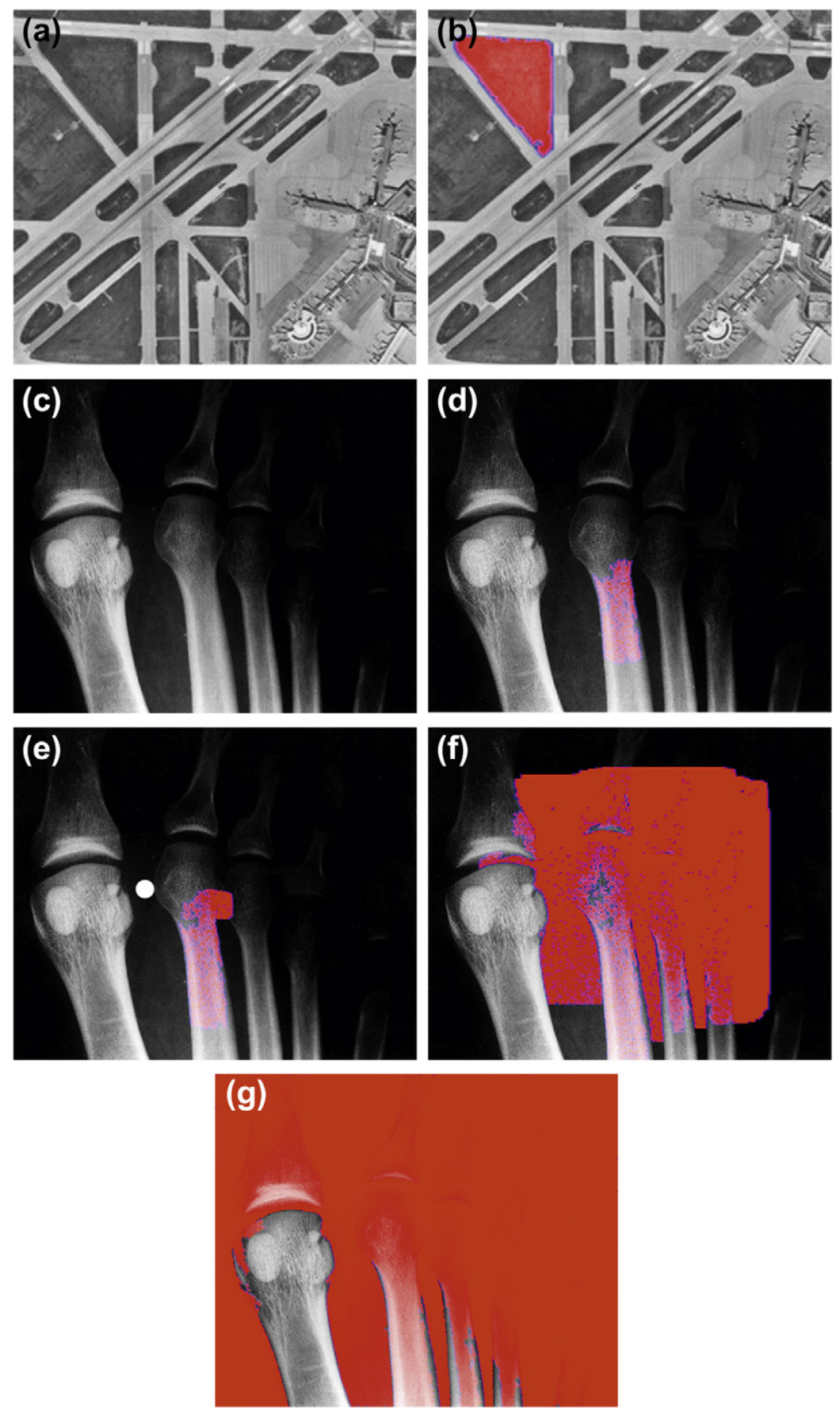

PLATE 3 (Figure 3 on page 10 of this volume) 
(a)

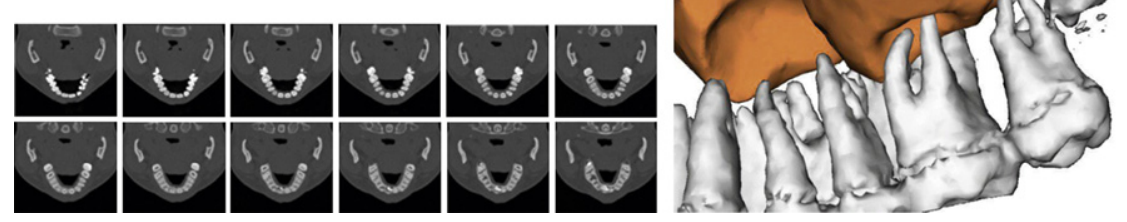

PLATE $4 b$ (Figure $4 b$ on page 12 of this volume)

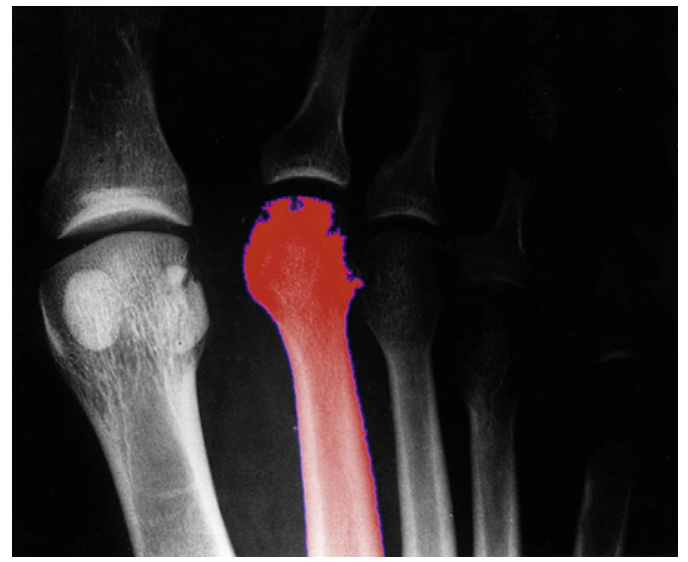

PLATE 5 (Figure 5 on page 12 of this volume)

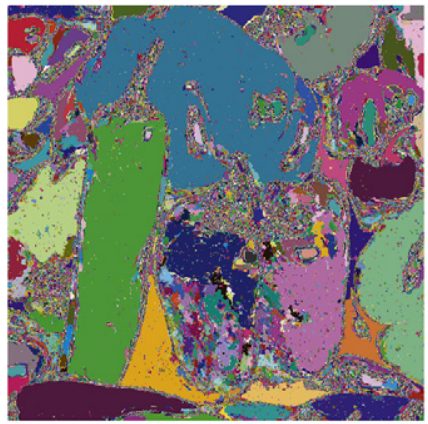

(a)

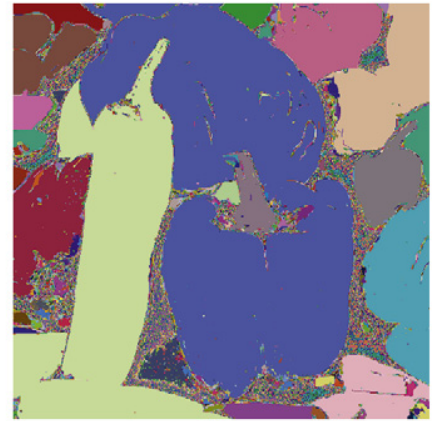

(b)

PLATE 15 (Figure 15 on page 22 of this volume) 


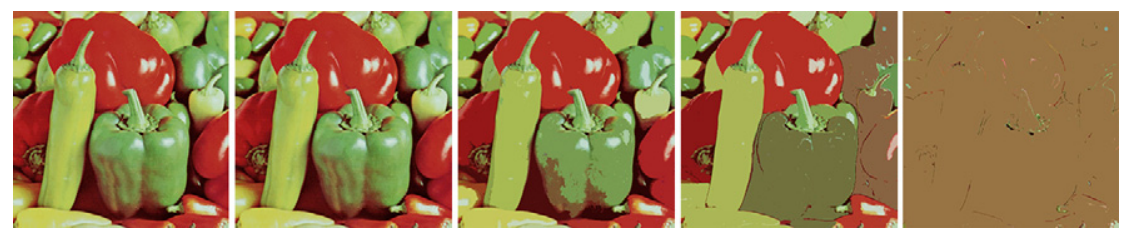

PLATE 16 (Figure 16 on page 22 of this volume)
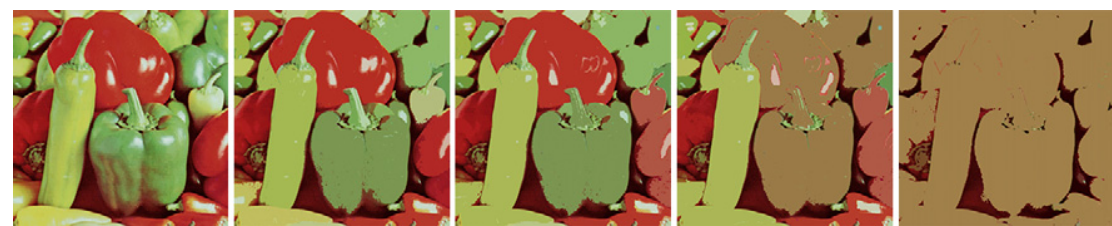

PLATE 17 (Figure 17 on page 23 of this volume)

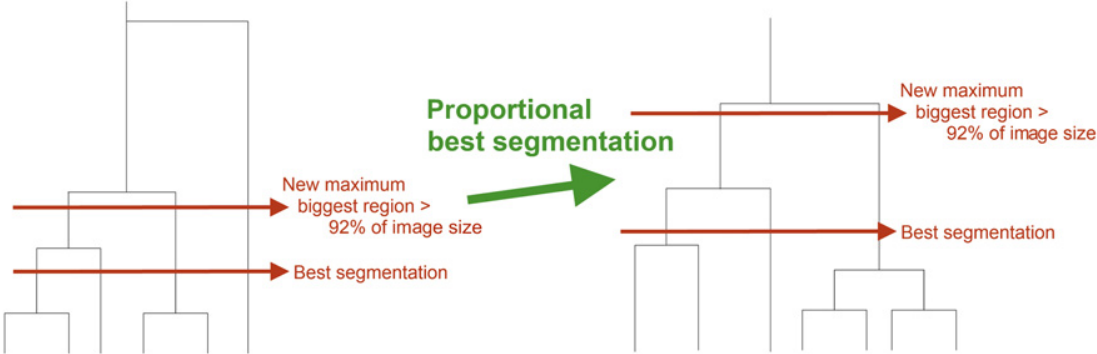

PLATE 18 (Figure 18 on page 23 of this volume)

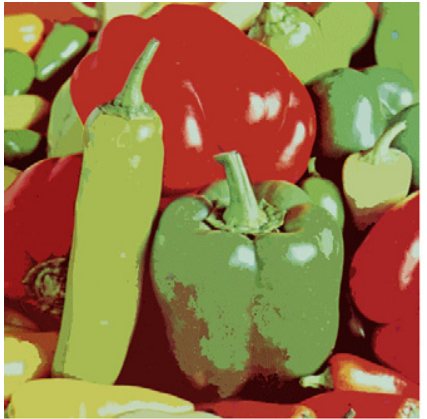

(a)

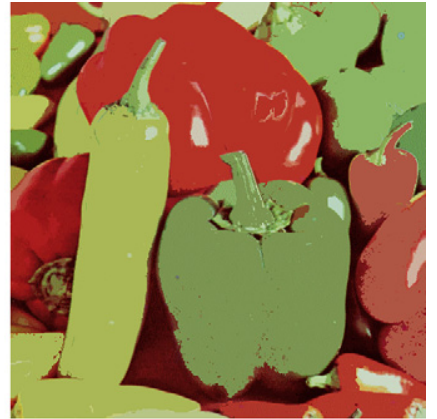

(b)

PLATE 19 (Figure 19 on page 23 of this volume) 


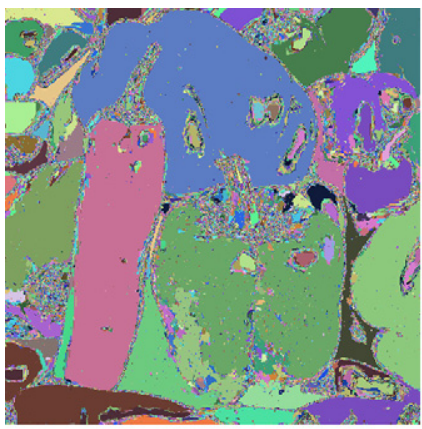

(a)

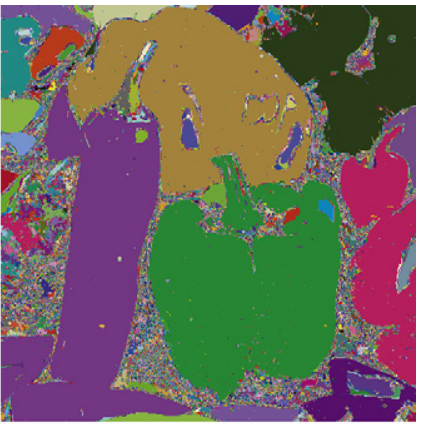

(b)

PLATE 20 (Figure 20 on page 24 of this volume)

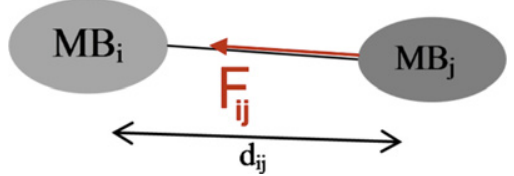

PLATE 22 (Figure 22 on page 25 of this volume)

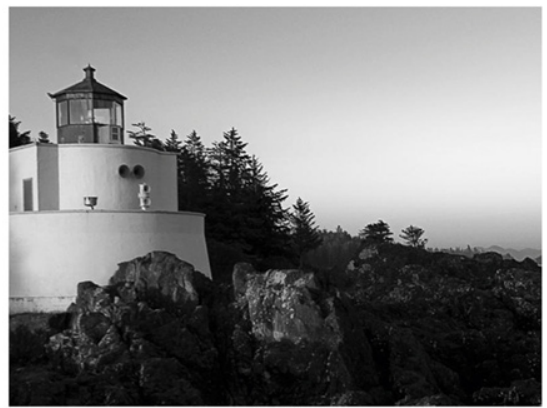

(a)

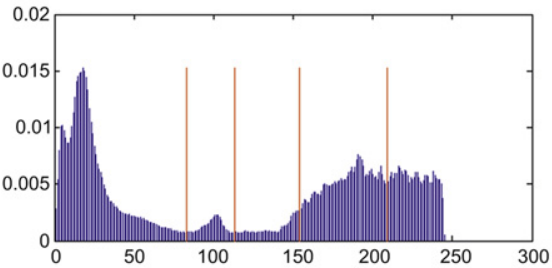

(b)

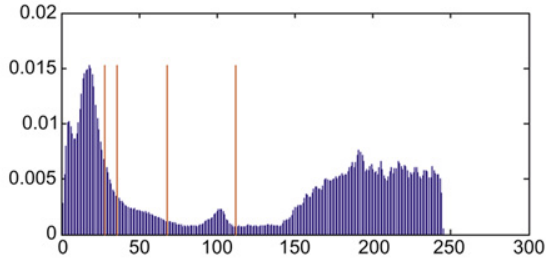

(c)

PLATE 27 (Figure 27 on page 34 of this volume) 


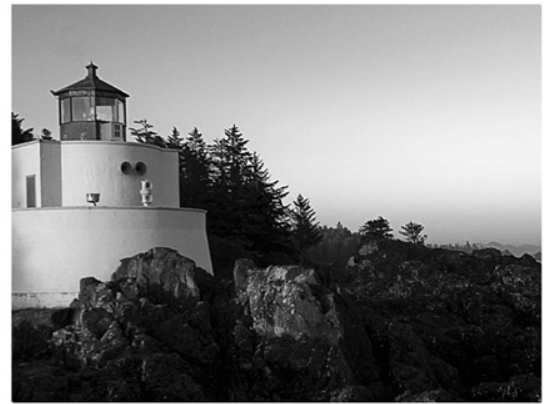

(a)

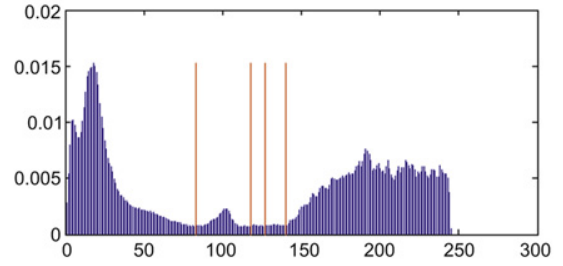

(b)

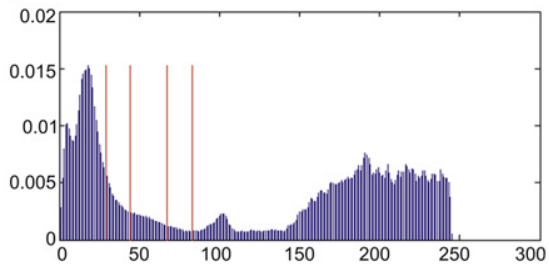

(c)

PLATE 29 (Figure 29 on page 36 of this volume)

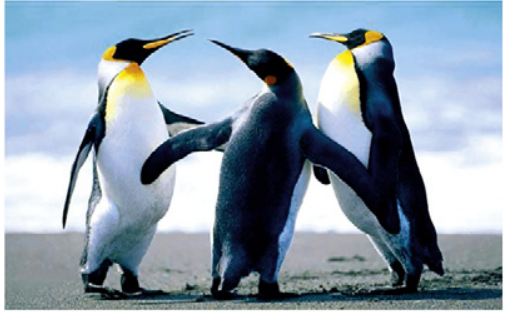

(a)

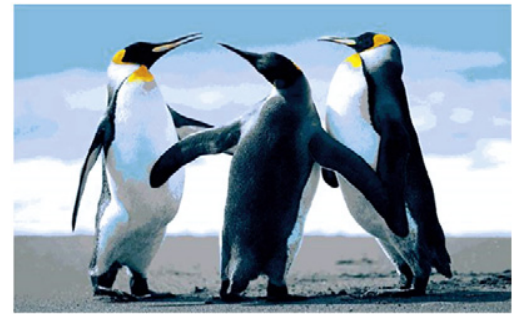

(b)

PLATE 33 (Figure 33 on page 39 of this volume) 


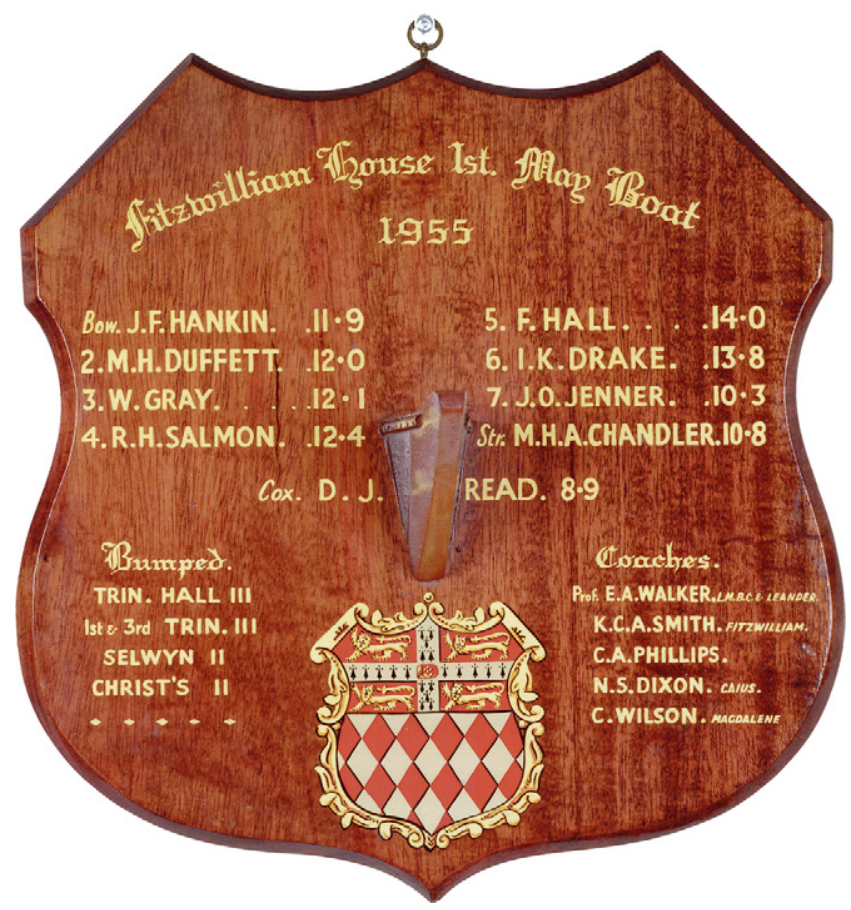

PLATE 6 (Figure 6 on page 207 of this volume)

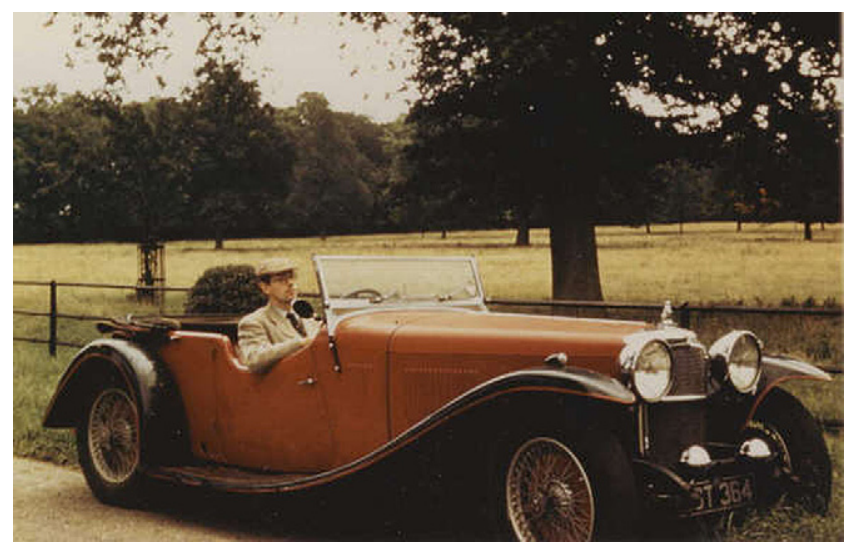

PLATE 16 (Figure 16 on page 230 of this volume) 


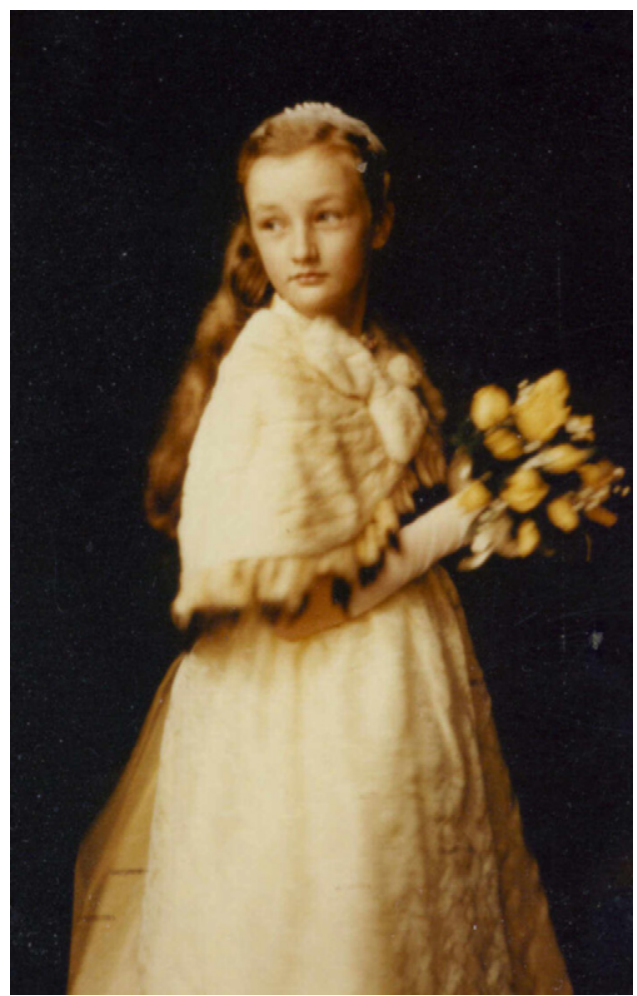

PLATE 18 (Figure 18 on page 233 of this volume)

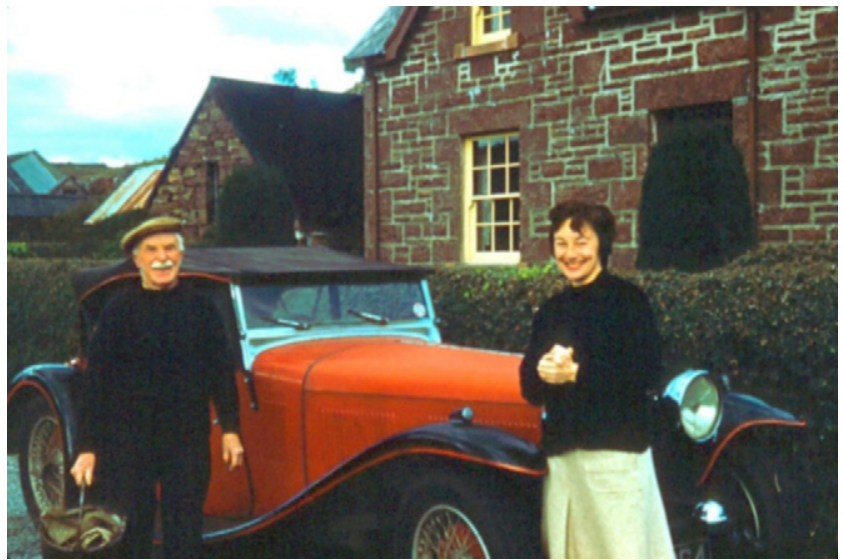

PLATE 19 (Figure 19 on page 235 of this volume) 


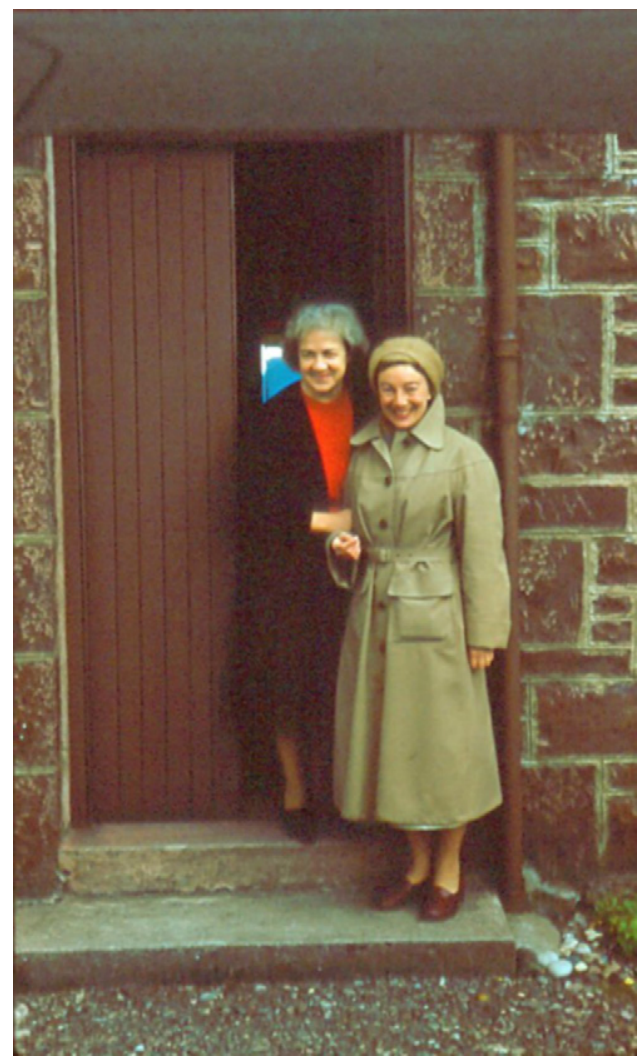

PLATE 20 (Figure 20 on page 235 of this volume)

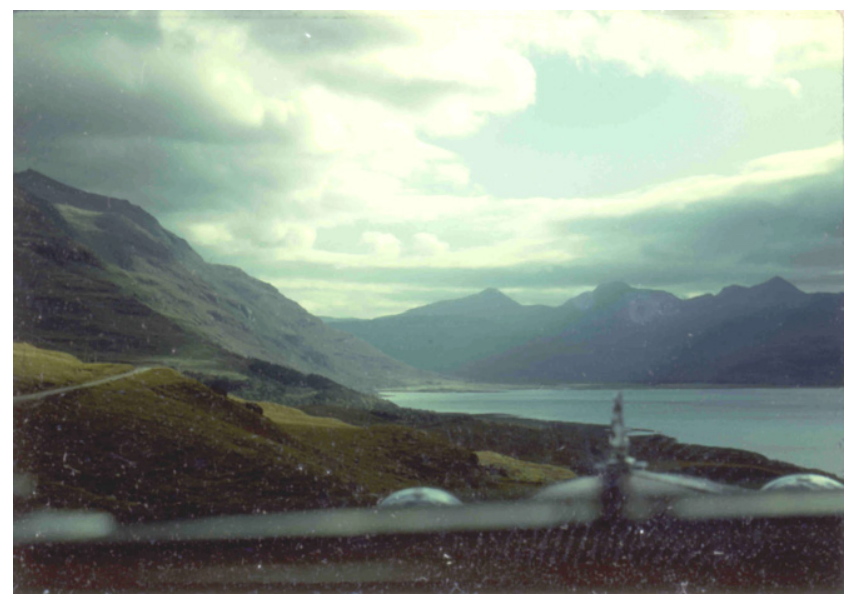

PLATE 21 (Figure 21 on page 236 of this volume) 


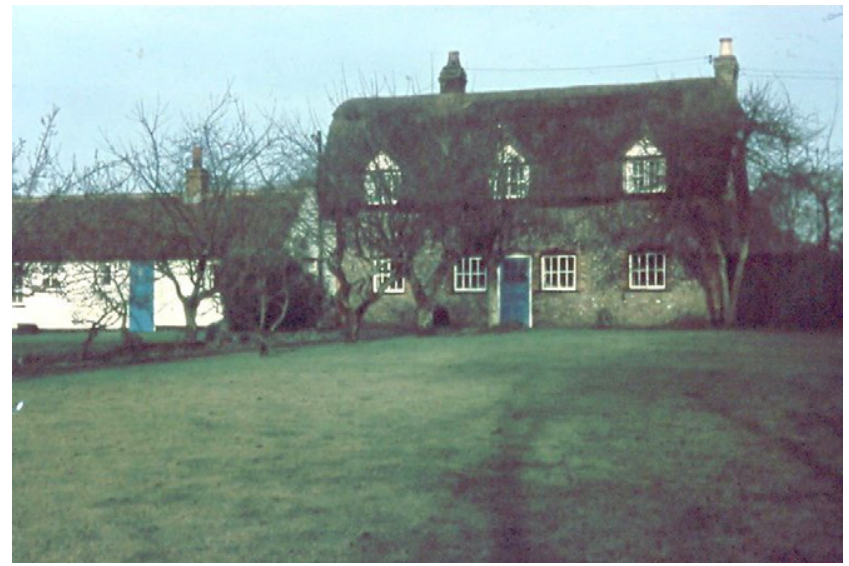

PLATE 24 (Figure 24 on page 252 of this volume)

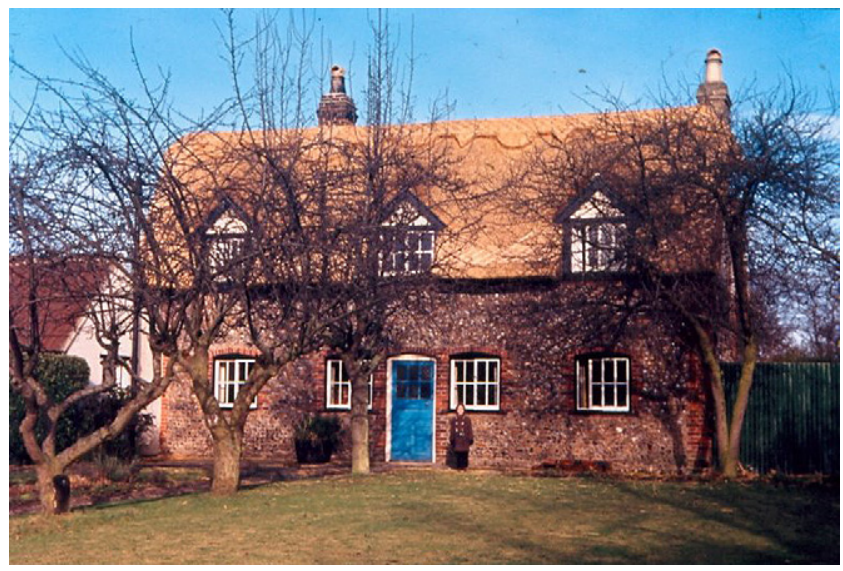

PLATE 25 (Figure 25 on page 252 of this volume) 


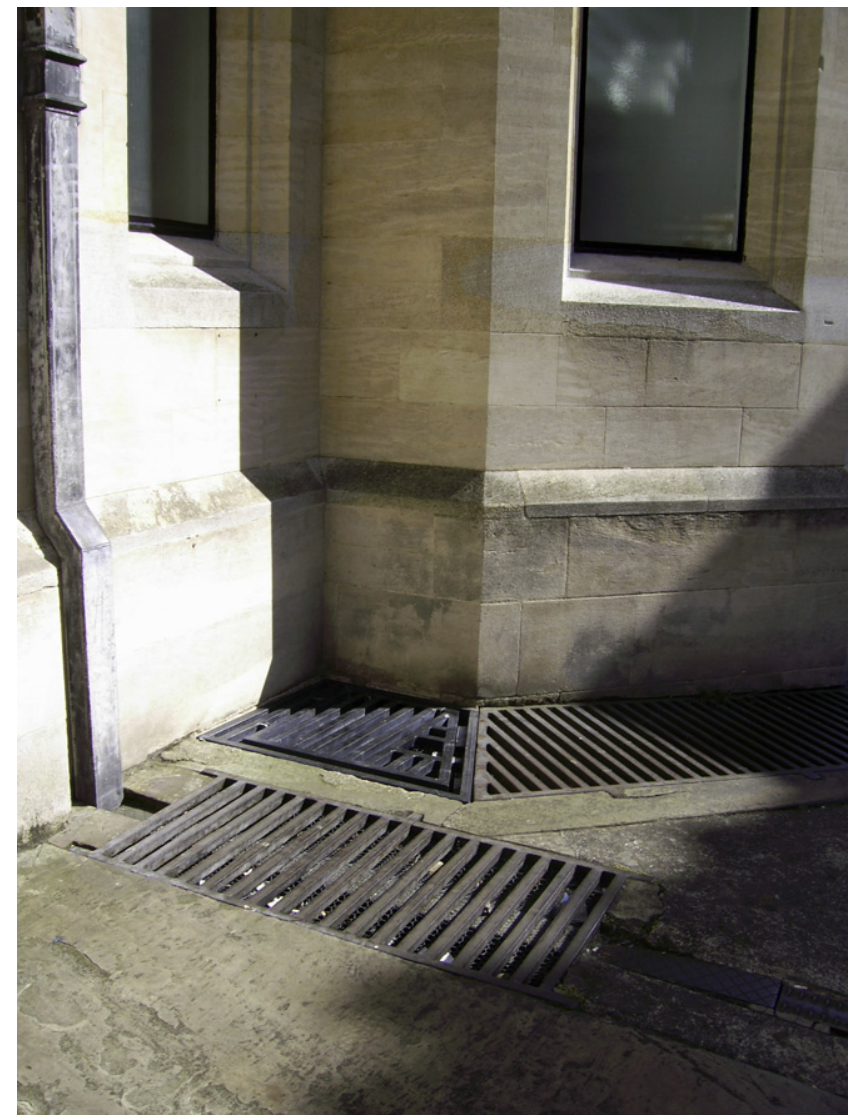

PLATE 28 (Figure 28 on page 256 of this volume) 


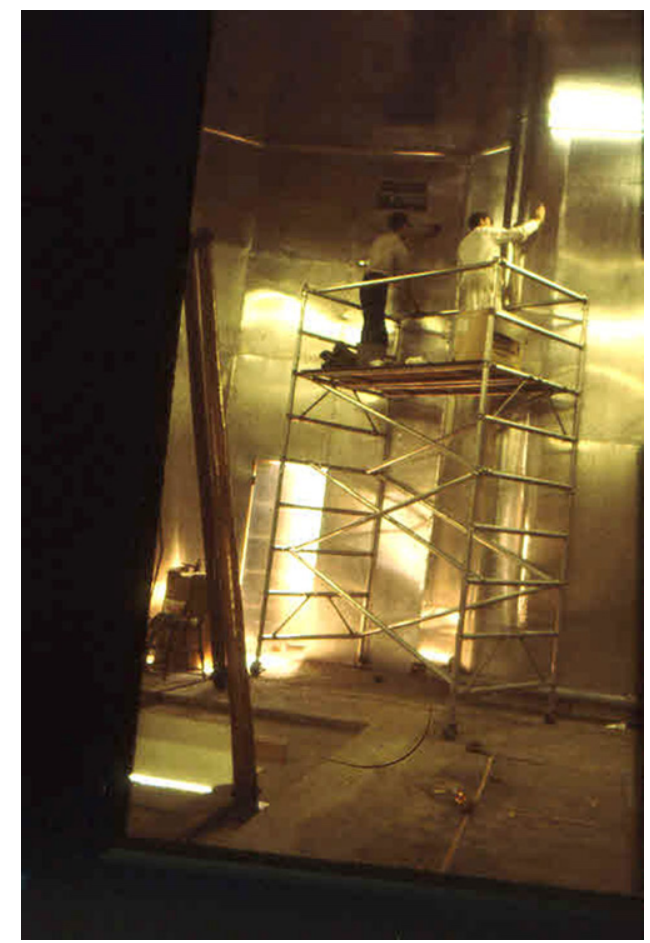

PLATE 29 (Figure 29 on page 260 of this volume)

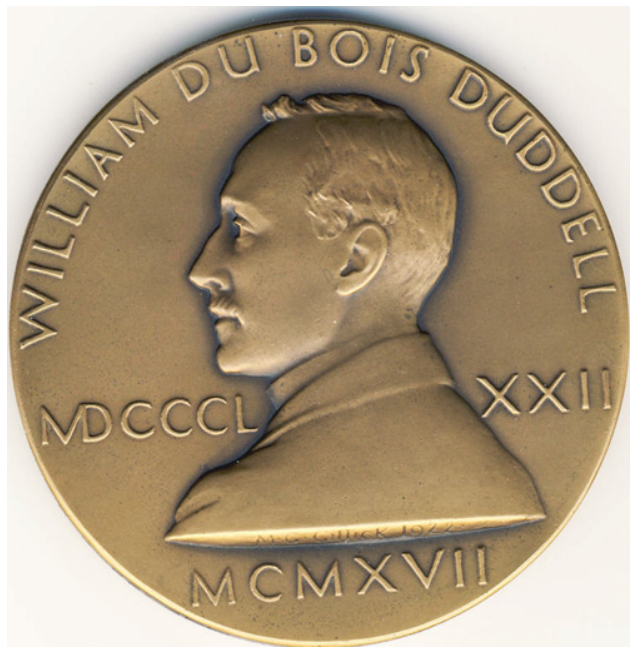

PLATE 32 (Figure 32 on page 271 of this volume) 

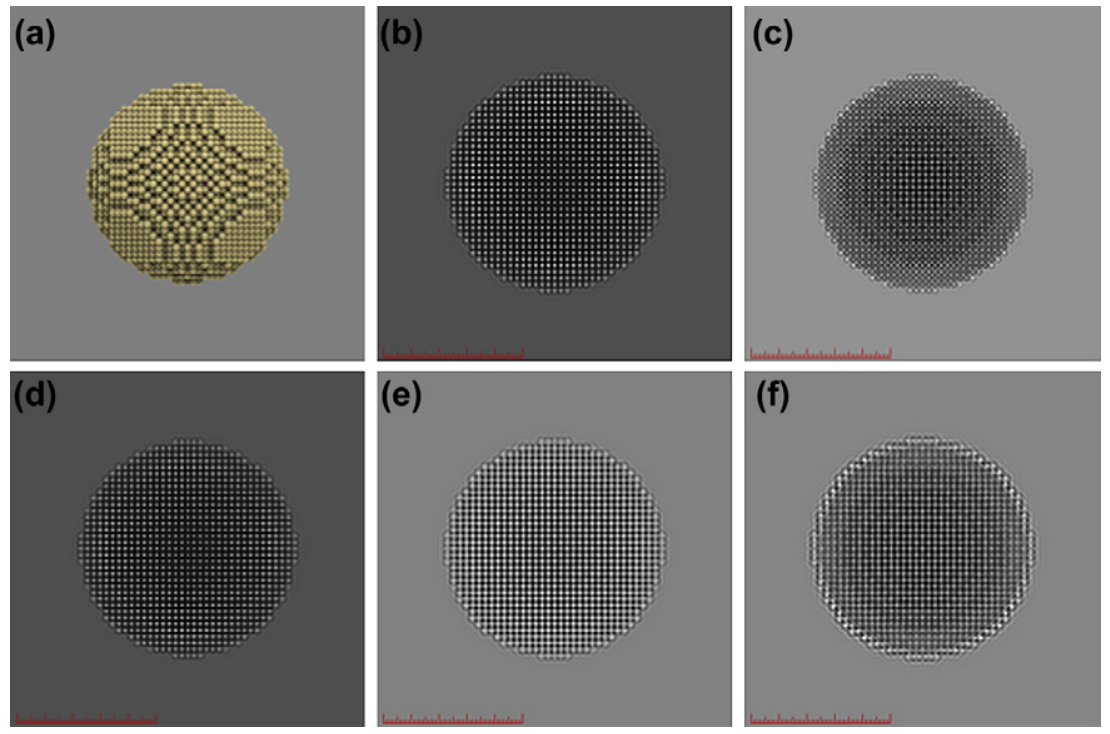

PLATE 4 (Figure 4 on page 288 of this volume) 
(a)

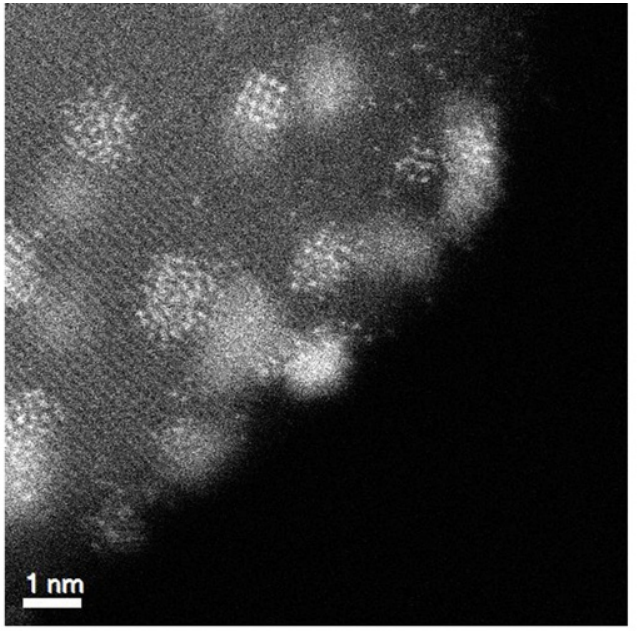

(b)

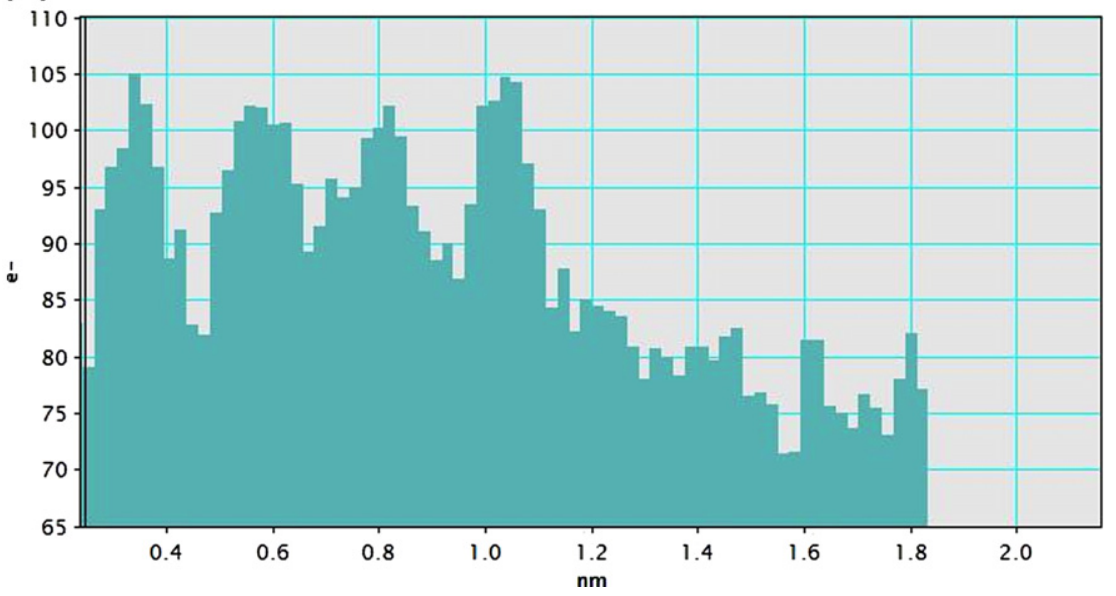

PLATE 12b (Figure 12b on page 296 of this volume) 


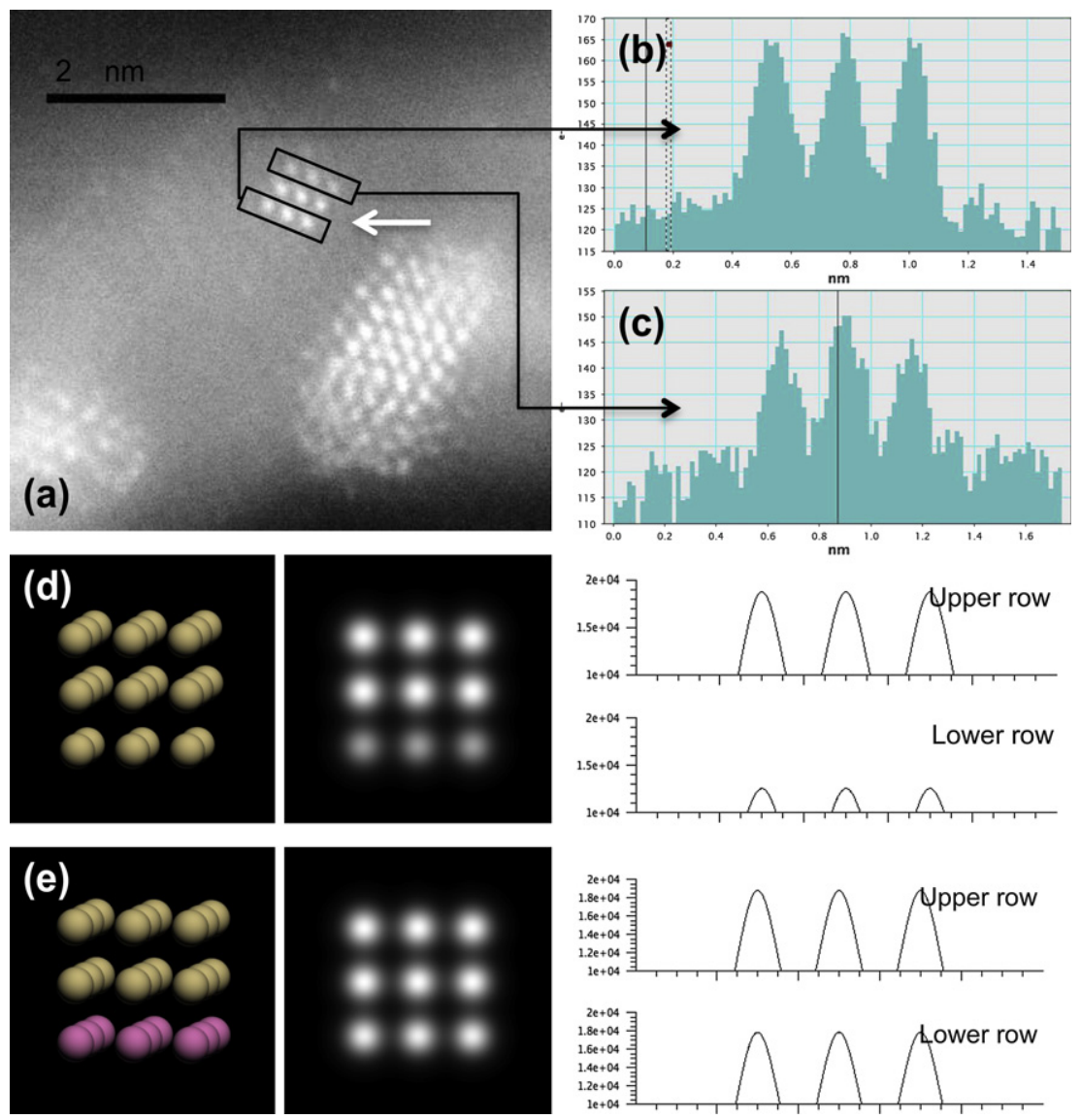

PLATE 14 (Figure 14 on page 298 of this volume)

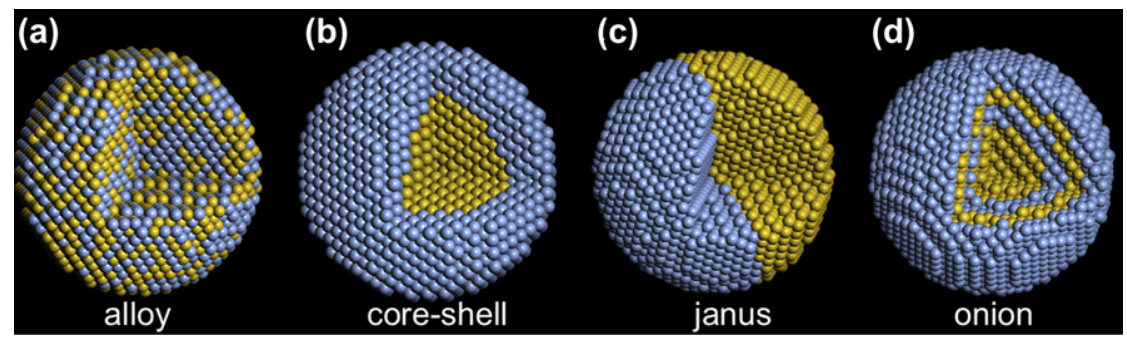

PLATE 19 (Figure 19 on page 305 of this volume) 


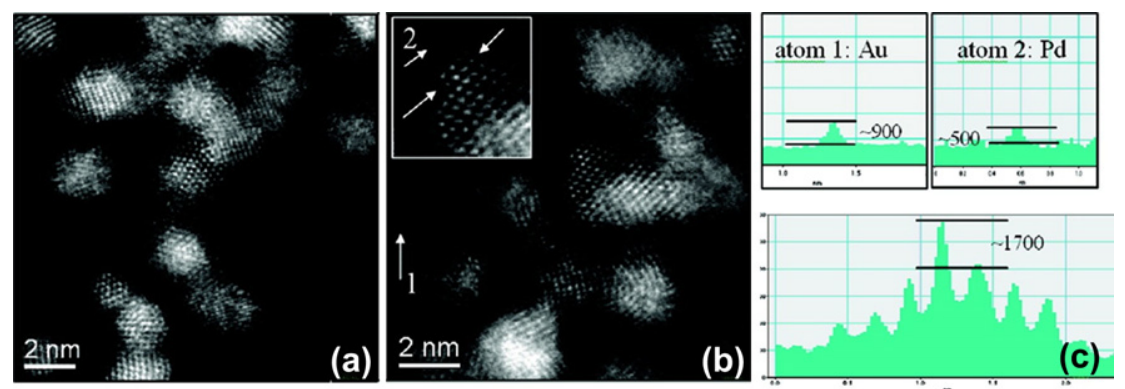

PLATE 20 (Figure 20 on page 306 of this volume)

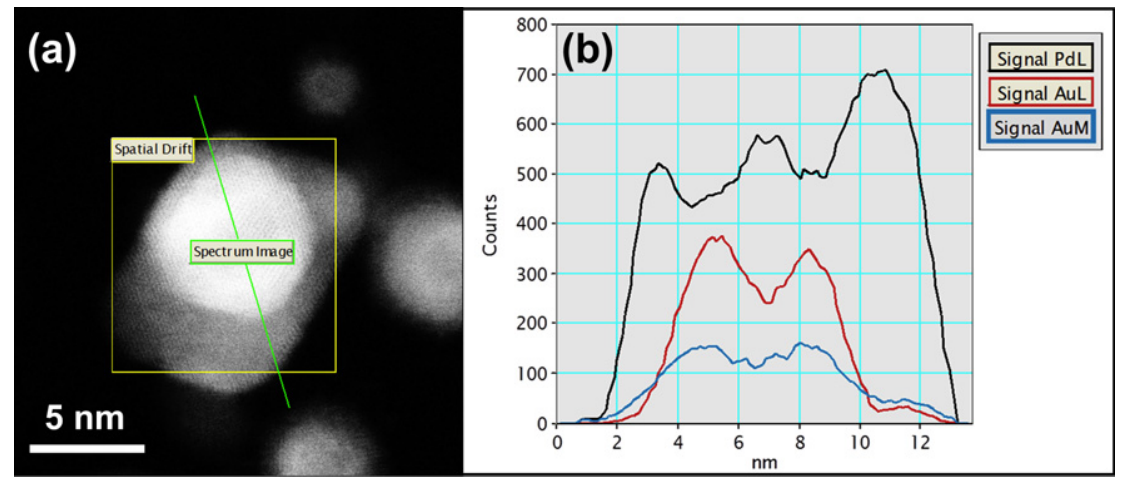

PLATE 22 (Figure 22 on page 308 of this volume)
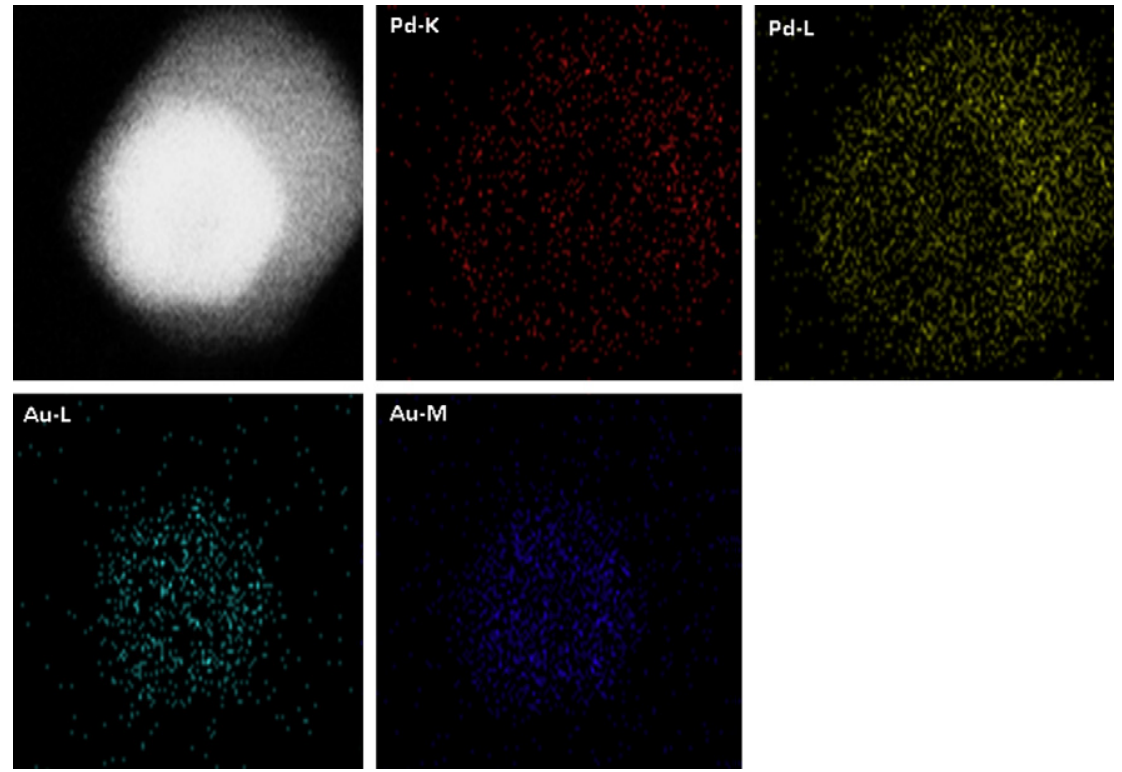

PLATE 23 (Figure 23 on page 309 of this volume) 


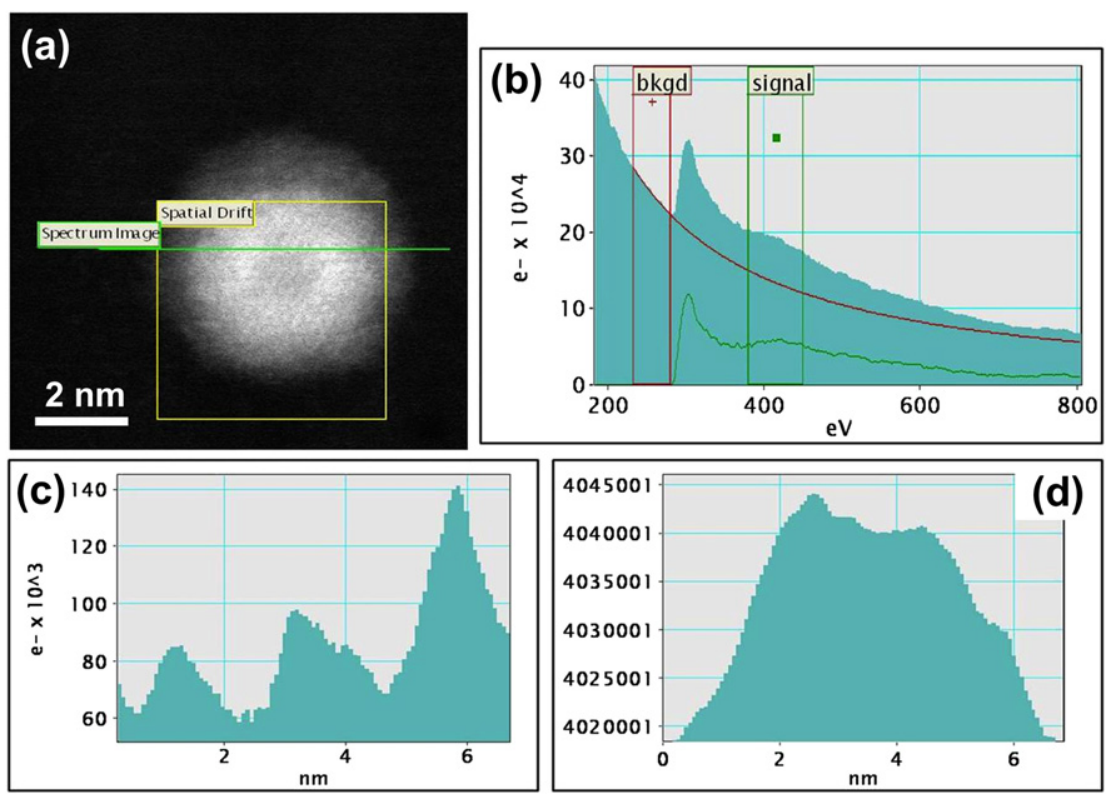

PLATE 24 (Figure 24 on page 310 of this volume) 

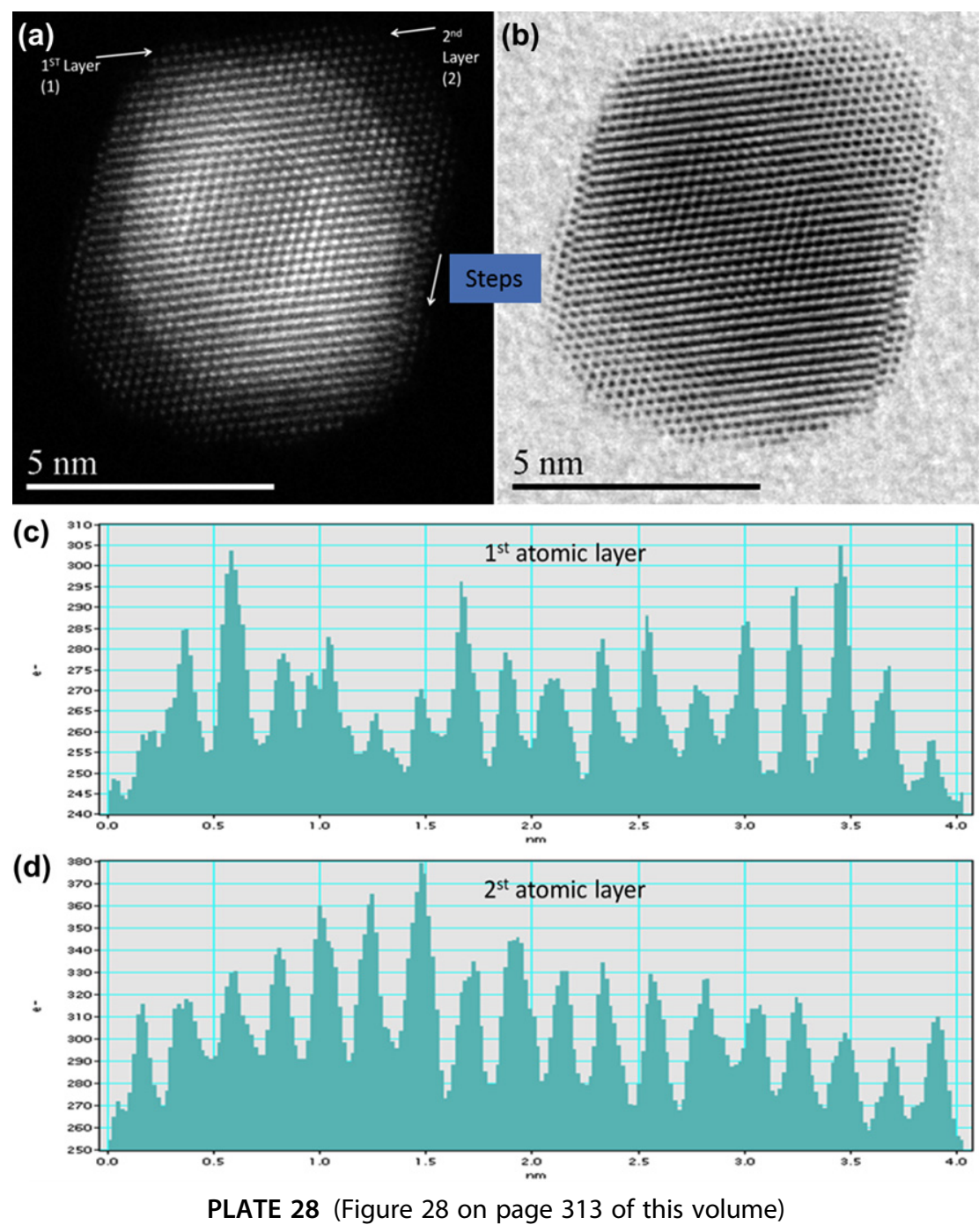

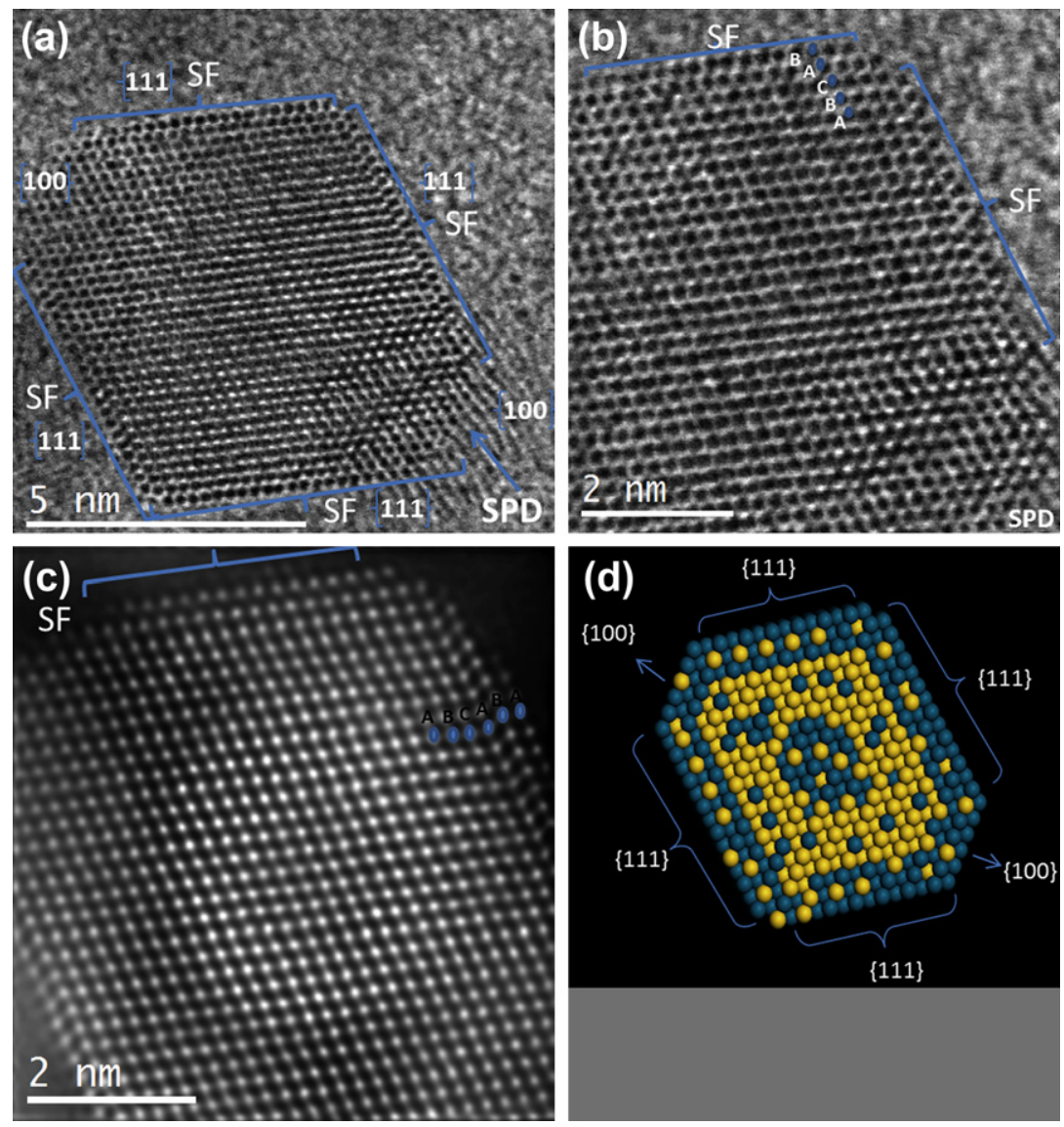

PLATE 29 (Figure 29 on page 314 of this volume) 

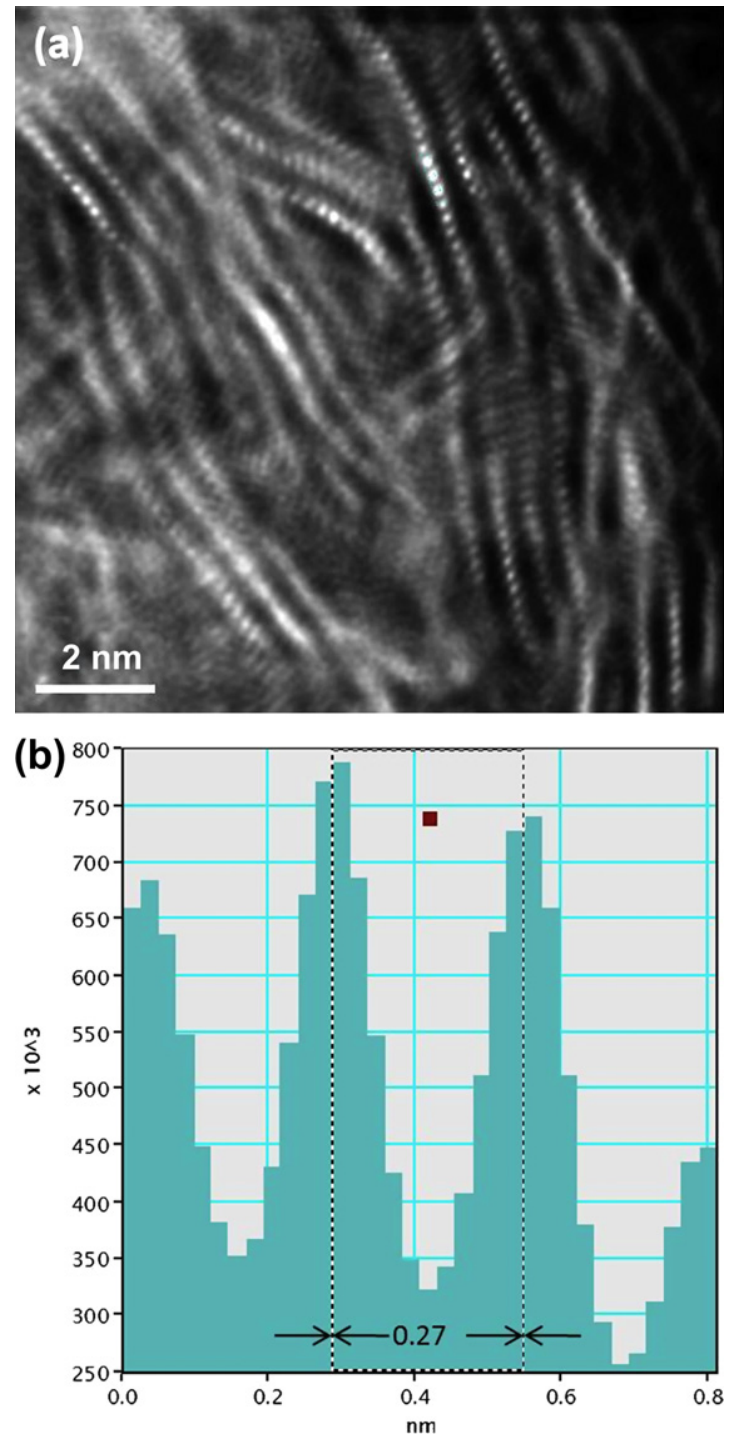

PLATE 34 (Figure 34 on page 322 of this volume) 

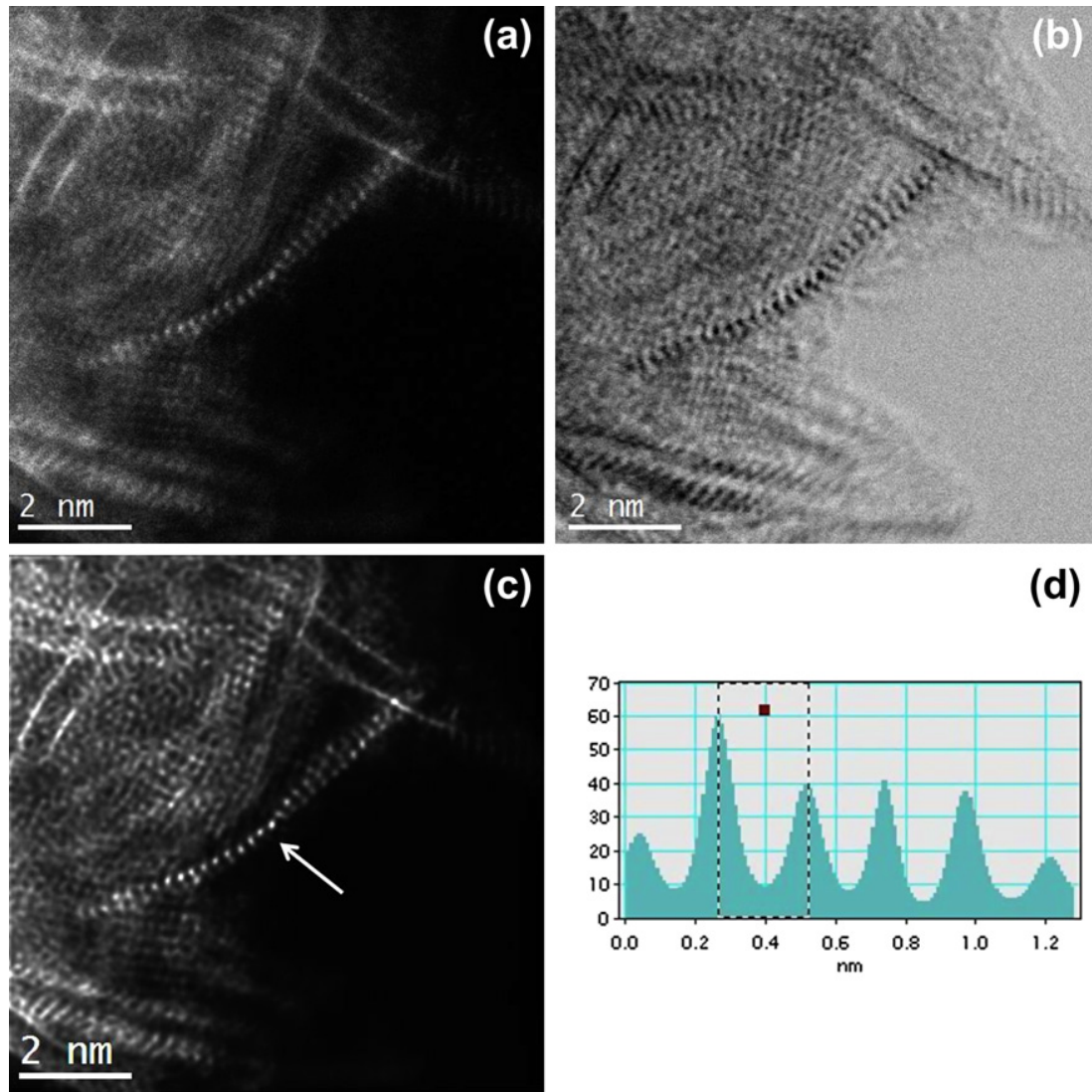

(d)

PLATE 35 (Figure 35 on page 323 of this volume)

(a)

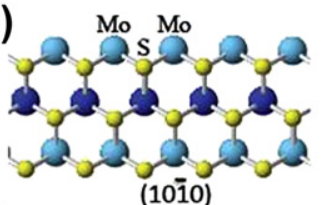

(c)

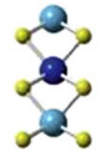

(b)

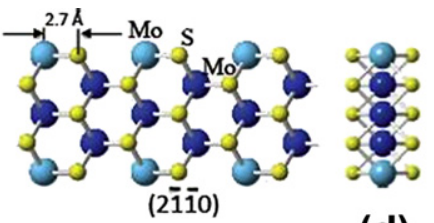

$\mathrm{S}$

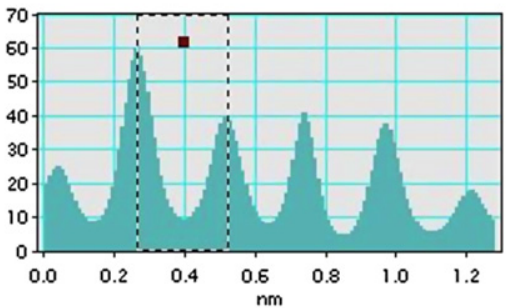

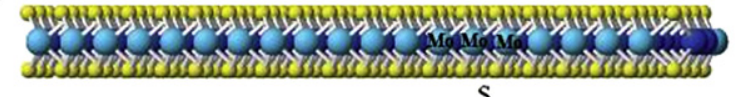

$\mathrm{S}$

(e)

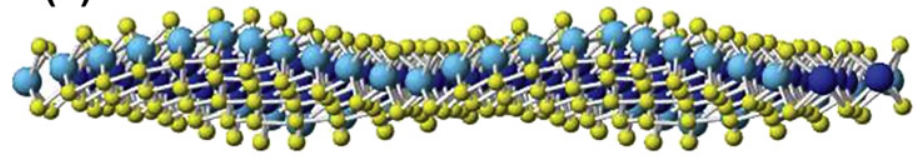

(g)

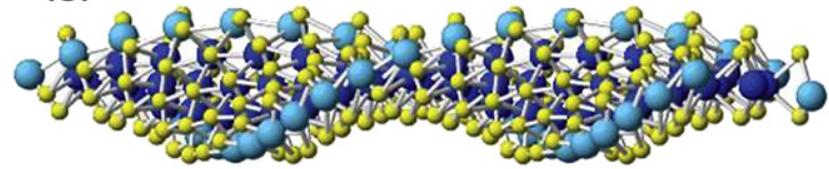

(d)

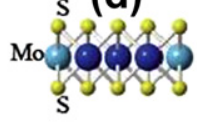

(f)

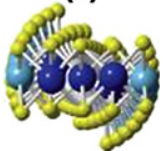

(h)

PLATE 36 (Figure 36 on page 324 of this volume) 

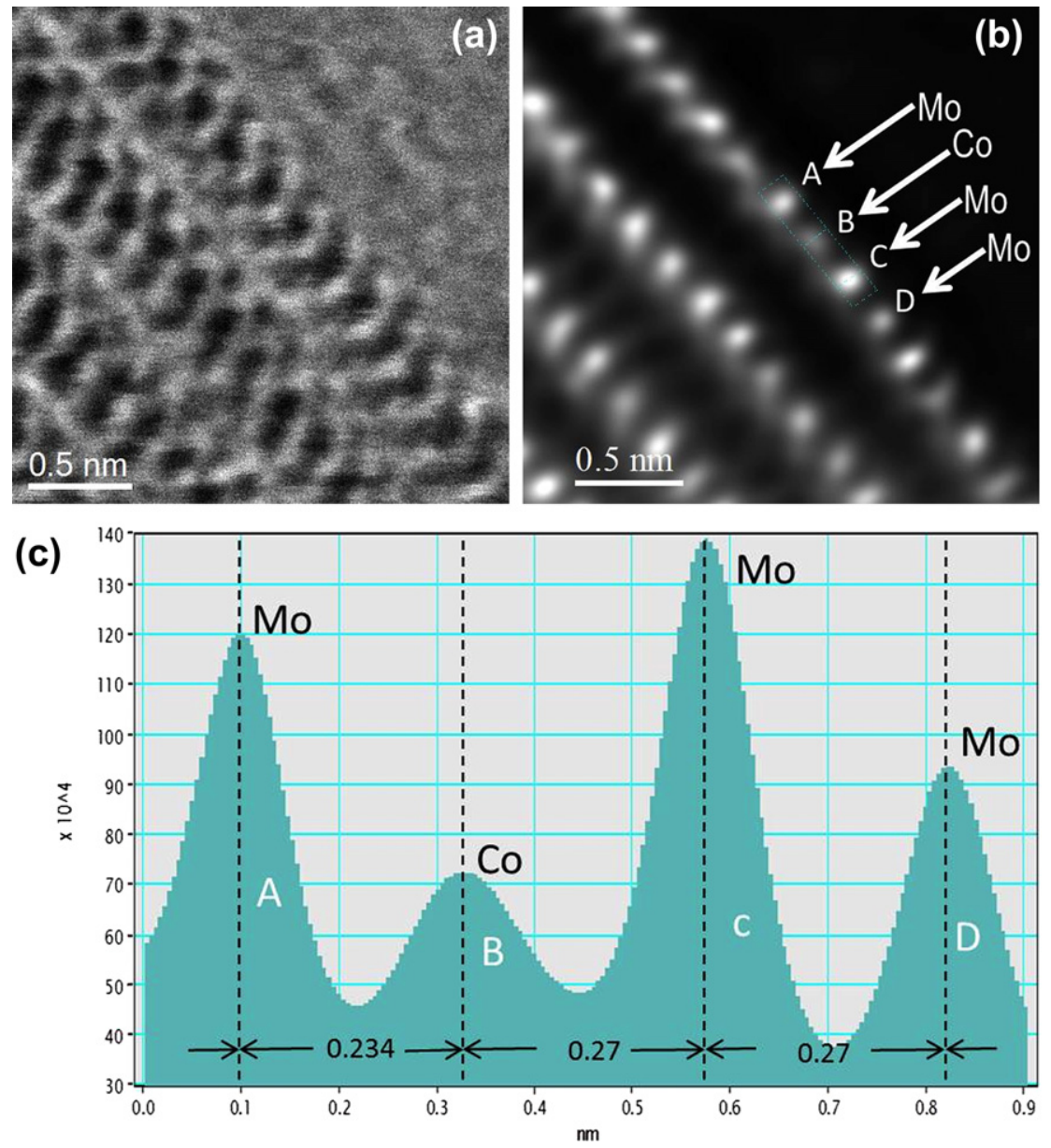

PLATE 38 (Figure 38 on page 328 of this volume) 

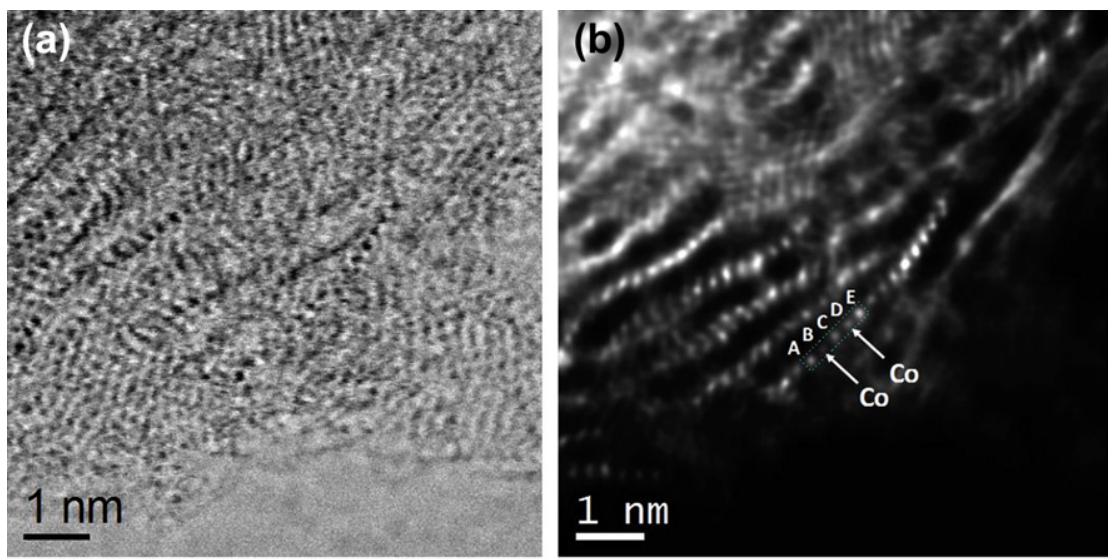

(c)

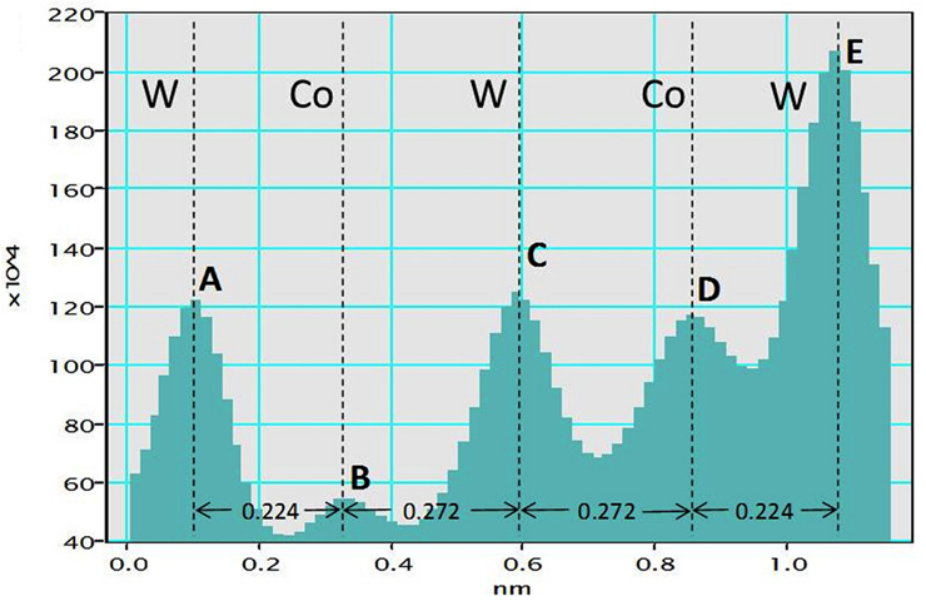

PLATE 40 (Figure 40 on page 330 of this volume)
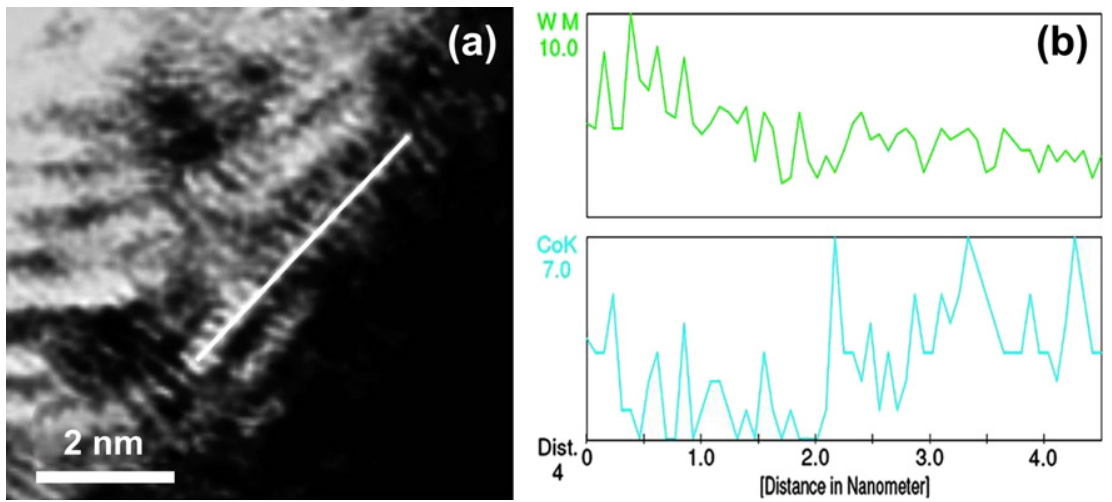

PLATE 41 (Figure 41 on page 331 of this volume) 


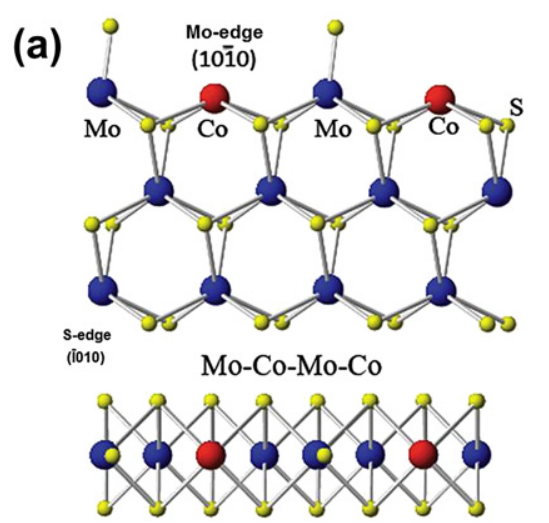

Mo-edge

(c) $\begin{aligned} & \text { Mo-edge } \\ & (10 \overline{10})\end{aligned}$

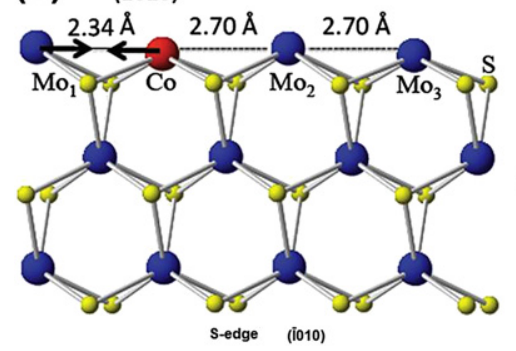

(b)
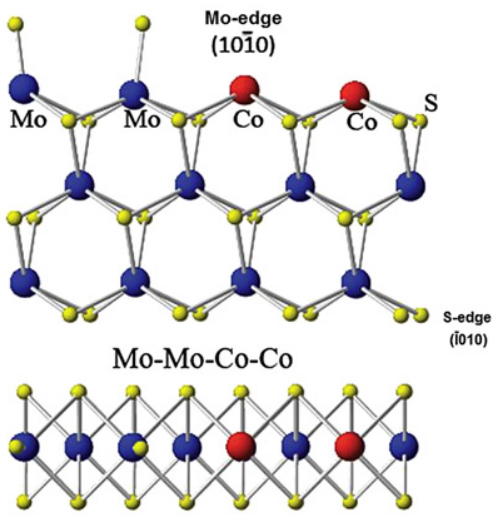

Mo-edge

(d) $\begin{gathered}\text { Wedge } \\ (1010)\end{gathered}$

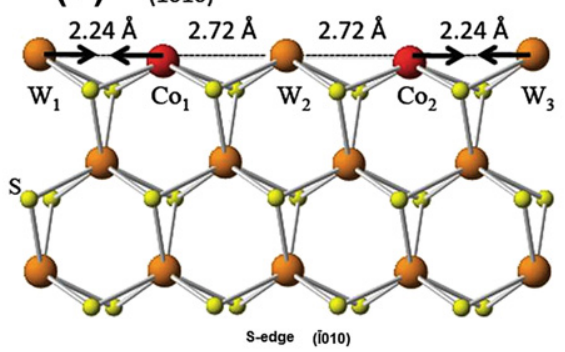

PLATE 42 (Figure 42 on page 332 of this volume) 